\title{
CONVERGENCE OF LOCAL TYPE DIRICHLET FORMS TO A NON-LOCAL TYPE ONE
}

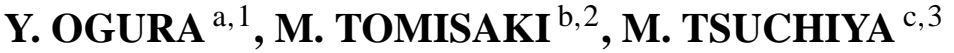 \\ ${ }^{a}$ Department of Mathematics, Saga University, Saga, Japan \\ ${ }^{\mathrm{b}}$ Department of Mathematics, Nara Women's University, Nara, Japan \\ ${ }^{\mathrm{c}}$ Department of Mechanical Engineering,Kanazawa University, Kanazawa, Japan
}

Received 4 December 2000, revised 16 May 2001

\begin{abstract}
Convergence of Dirichlet forms of diffusion processes is investigated without assuming that the underlying measures are fixed or compatible with a fixed one. Here we treat the case where the basic processes are skew product of finite dimensional diffusions and onedimensional ones. We note the corresponding diffusions to the Dirichlet forms can be represented as time changed processes of the basic processes, where the time change is given by the additive functional associated with the underlying measure. Then the convergence of the Markov semigroups of the obtained processes and the Feller property of the limit process are proved by providing some convergence properties on additive functionals. The concrete expression on a core for the limit Dirichlet form is also obtained, which may be of non-local type due to the degeneracy of the underlying measure. Finally, under some regularity assumption, the partial differential equation associated with the limit process is given, which is elliptic on infinitely many disjoint strips with the non-local boundary condition including the boundary values on the neighboring strips. @ 2002 Éditions scientifiques et médicales Elsevier SAS
\end{abstract}

RÉSUMÉ. - On étudie la convergence de la forme de Dirichlet des processus de diffusion sans l'hypothèse que les mesures de base sont fixées ou compatibles avec une mesure fixée. Nous traitons ici le cas où chaque processus fondamental est le produit semi-direct d'un processus de diffusion de dimension finie et d'un processus de diffusion unidimensionnel. Nous notons que les processus correspondants à la forme de Dirichlet peuvent être représentés comme des processus changés de temps des processus fondamentaux. Le changement de temps est donné par les fonctionnelles additives associées aux mesures de base. Nous démontrons la convergence des semi-groupes des processus markoviens ainsi obtenus et la propriété Fellerienne du processus limite en vérifiant la convergence des fonctionnelles additives. L'expression concrète de la forme de Dirichlet sur un domaine dense est aussi obtenue. Elle peut être de type non local du fait de la dégénérescence de la mesure de base du processus limite. Enfin, sous une certaine hypothèse sur

E-mail addresses: ogura@ms.saga-u.ac.jp (Y. Ogura), tomisaki@cc.nara-wu.ac.jp (M. Tomisaki), tsuchiya@kenroku.kanazawa-u.ac.jp (M. Tsuchiya).

${ }^{1}$ Research supported by Grant-in-Aid for Scientific Research No. 11304003.

${ }^{2}$ Research supported by Grants-in-Aid for Scientific Research No. 11304003 and 12640125.

${ }^{3}$ Research supported by Grant-in-Aid for Scientific Research No. 10640112. 
régularité, nous présentons l'équation aux derivées partielles associée au processus limite. Il est elliptique sur une infinité de bandes deux à deux disjointes et satisfait à une condition au bord sur la frontière de chaque bande, en fonction des valeurs sur les bandes voisines. ๑ 2002 Éditions scientifiques et médicales Elsevier SAS

\section{Introduction}

In the study of convergence of a sequence of Markov processes, the theory of Dirichlet forms is one of the most useful tools. In fact, the notion of $\Gamma$-convergence and Mosco convergence as well as a monotone convergence theorem derive a lot of interesting results (see [2,15,17,12,10,14,20], etc.). However, they are basically for a sequence of Markov processes associated with Dirichlet forms on a fixed underlying Hilbert space, which means that the underlying measures are fixed or at least compatible with a fixed one.

In this article, we will be concerned with a sequence of diffusion processes whose underlying measures of Dirichlet forms converge to a degenerate one. Especially, we are interested in the case where a sequence of diffusion processes converges to a nonlocal Markov process, which never happens unless the underlying measures degenerate. As a prototype of such sequences, we adopt that of diffusion processes which are skew products of finite dimensional diffusion processes and one-dimensional ones. This enables us to express the semigroups as time changed processes of product diffusions and obtain some properties such as convergence of semigroups and Feller property of the limit process. The feature of the Dirichlet forms with degenerate underlying measures and Dirichlet energy are already given in [7] using harmonic operators. We would notice that those are actually expressed as a sum of local Dirichlet forms on the support of the underlying measure and non-local Dirichlet forms on its boundaries. We will give the explicit form of the Lévy measure for the Dirichlet form obtained as a limit of diffusion processes in our setting (see Theorem 5.1 below). As is noted in [18] and [19], the Dirichlet form of the type thus obtained corresponds to the second order differential equation with non-local type boundary condition. Our simpler proof of Feller property here offers another approach to this problem, which is actually an extension of the results in [8]. However we must confess that this is fully based on the skew product structure, and the rather tedious real analysis might be on stage if one deal with more general processes. Finally, we would say that the results even in our case give a new type of phenomena of the convergence of Dirichlet forms and well provide the rough shape of formulas in forthcoming general settings.

This article is organized as follows. In the next Section 2, we set up our problem in terms of Dirichlet forms and give an intuitive scope of our limit theorem. We establish our limit theorem in Section 3, where we express the processes in terms of time change by additive functionals and prove their convergence. In Section 4, we show the Feller property of the semigroup of the limit process, using the expression of one-dimensional diffusion processes by a Brownian motion and its local time. In Section 5, we give the explicit form of the Dirichlet form associated with the limit process as a sum of local and non-local Dirichlet forms under a little stronger conditions. We also give several examples, in which we can compute the explicit formulas of Lévy measures and 
determine the domain of the Dirichlet forms except for the last one. In the final Section 6, we study the partial differential equations for the resolvent of the limit process. The obtained one is elliptic on infinitely many disjoint strips with the non-local boundary condition including the boundary values on the neighboring strips.

\section{Preliminaries}

For $E=\mathbb{R}^{d}$ or $E=\mathbb{R}^{d-1}$, we denote by $C(E)$ the space of all bounded continuous functions on $E$, and by $\widehat{C}(E)$ the space of those functions in $C(E)$ which vanish at infinity. The set of all infinitely differentiable functions on $E$ with compact support is denoted by $C_{0}^{\infty}(E)$, while the Sobolev space of order one by $H^{1}(E)$. We also write as $x=\left(x^{1}, \ldots, x^{d-1}, x^{d}\right)=\left(x^{\prime}, x^{d}\right) \in \mathbb{R}^{d-1} \times \mathbb{R}$, and denote by $\mathrm{d} x$, $\mathrm{d} x^{\prime}, \mathrm{d} \xi$ the $d$-dimensional, $(d-1)$-dimensional, one-dimensional Lebesgue measure respectively.

Let $a^{i j}=a^{i j}\left(x^{\prime}\right), i, j=1,2, \ldots, d-1$, be a system of bounded measurable functions on $\mathbb{R}^{d-1}$ which compose a symmetric and uniformly elliptic matrix $\left(a^{i j}\right)_{i, j=1,2, \ldots, d-1}$. Let also $a^{d d}=a^{d d}(\xi)$ be a bounded measurable function which is bounded from below by a positive constant.

For each $n \in \mathbb{N}$, let $\rho_{0}^{(n)}=\rho_{0}^{(n)}(\xi)$ be a nonnegative bounded measurable function on $\mathbb{R}$ and $\mu_{0}^{(n)}=\mu_{0}^{(n)}(x)$ a bounded measurable function on $\mathbb{R}^{d}$ which are bounded from below by a positive constant. We now let $\mu^{(n)}(\mathrm{d} x)=\mu_{0}^{(n)}(x) \mathrm{d} x$ and consider the Dirichlet form $\left(\mathcal{E}^{(n)}, \mathcal{F}^{(n)}\right)$ on $L^{2}\left(\mathbb{R}^{d}, \mu^{(n)}\right)$ given by

$$
\begin{gathered}
\mathcal{E}^{(n)}(u, v)=\frac{1}{2} \int_{\mathbb{R}^{d}}\left\{\left(1+\rho_{0}^{(n)}\left(x^{d}\right)\right) \sum_{i, j=1}^{d-1} a^{i j}\left(x^{\prime}\right) \partial_{x^{i}} u(x) \partial_{x^{j}} v(x)\right. \\
\left.+a^{d d}\left(x^{d}\right) \partial_{x^{d}} u(x) \partial_{x^{d}} v(x)\right\} \mathrm{d} x, \\
\mathcal{F}^{(n)}=H^{1}\left(\mathbb{R}^{d}\right),
\end{gathered}
$$

where the derivatives $\partial_{x^{i}} u:=\partial u / \partial x^{i}, 1 \leqslant i \leqslant d$, are taken in the distribution sense. It then follows that the Dirichlet form $\left(\mathcal{E}^{(n)}, \mathcal{F}^{(n)}\right)$ on $L^{2}\left(\mathbb{R}^{d}, \mu^{(n)}\right)$ is regular, so that there exists an associated Hunt process $\mathbf{X}^{(n)}$ on $\mathbb{R}^{d}$ (see [7]). It is also known that the associated semigroup $\left\{p_{t}^{(n)}: t>0\right\}$ on $L^{2}\left(\mathbb{R}^{d}, \mu^{(n)}\right)$ is Markovian in the sense that $0 \leqslant p_{t}^{(n)} f \leqslant 1$, a.e. whenever $0 \leqslant f \leqslant 1$, a.e. ([7]).

Proposition 2.1. - For each $n \in \mathbb{N},\left\{p_{t}^{(n)}: t>0\right\}$ is a Feller semigroup on $\widehat{C}\left(\mathbb{R}^{d}\right)$. Namely,

$$
\begin{gathered}
p_{t}^{(n)}\left(\widehat{C}\left(\mathbb{R}^{d}\right)\right) \subset \widehat{C}\left(\mathbb{R}^{d}\right), \quad t>0, \\
\limsup _{t \downarrow 0}\left|p_{x \in \mathbb{R}^{d}}^{(n)} f(x)-f(x)\right|=0, \quad f \in \widehat{C}\left(\mathbb{R}^{d}\right) .
\end{gathered}
$$

There exists a conservative diffusion process $\mathbf{X}^{(n)}=\left(X_{t}^{(n)}, P_{x}^{(n)}\right)$ on $\mathbb{R}^{d}$ which has $\left\{p_{t}^{(n)}, t>0\right\}$ as the transition operator, that is, 


$$
p_{t}^{(n)} f(x)=E_{x}^{(n)}\left[f\left(X_{t}^{(n)}\right)\right], \quad t>0, x \in \mathbb{R}^{d}, f \in \widehat{C}\left(\mathbb{R}^{d}\right),
$$

where $E_{x}^{(n)}$ stands for the expectation with respect to $P_{x}^{(n)}$.

Proof. - Under the assumptions, $L^{2}\left(\mathbb{R}^{d}, \mu^{(n)}\right)=L^{2}\left(\mathbb{R}^{d}, \mathrm{~d} x\right)$ and the space $\{u \in$ $\left.L^{2}\left(\mathbb{R}^{d}, \mu^{(n)}\right): \partial_{x^{i}} u \in L^{2}\left(\mathbb{R}^{d}, \mathrm{~d} x\right), 1 \leqslant i \leqslant d\right\}$ coincides with the Sobolev space $H^{1}\left(\mathbb{R}^{d}\right)$. Further, the norm $\mathcal{E}_{1}^{(n)}(,)^{1 / 2}$ is equivalent to the norm \|\|$_{H^{1}\left(\mathbb{R}^{d}\right)}$, where $\mathcal{E}_{\lambda}^{(n)}(u, v)=$ $\mathcal{E}^{(n)}(u, v)+\lambda(u, v)_{L^{2}\left(\mathbb{R}^{d}, \mu^{(n)}\right)}, \quad \lambda>0$. Therefore we get the proposition by means of results due to Nash [16], Stampacchia [21] and Kunita [11].

In what follows, we consider the convergence of the diffusion processes $\mathbf{X}^{(n)}$ when $\rho_{0}^{(n)}(\xi) \mathrm{d} \xi$ converge to a measure $\rho(\mathrm{d} \xi)$ and $\mu_{0}^{(n)}(x) \mathrm{d} x$ to $\mu(\mathrm{dx})$ in some sense. The Dirichlet form $(\mathcal{E}, \mathcal{F})$ of the limit process could be supposed to be

$$
\begin{aligned}
\mathcal{E}(u, v)= & \frac{1}{2} \int_{\mathbb{R}^{d}} \sum_{i, j=1}^{d-1} a^{i j}\left(x^{\prime}\right) \partial_{x^{i}} u(x) \partial_{x^{j}} v(x) \mathrm{d} x^{\prime}\left(\mathrm{d} x^{d}+\rho\left(\mathrm{d} x^{d}\right)\right) \\
& +\frac{1}{2} \int_{\mathbb{R}^{d}} a^{d d}\left(x^{d}\right) \partial_{x^{d}} u(x) \partial_{x^{d}} v(x) \mathrm{d} x .
\end{aligned}
$$

However this is not right as it stands, since we must consider it on the space $L^{2}\left(\mathbb{R}^{d}, \mu\right)$ and the support of the measure $\mu$ is not necessarily equal to the whole space $\mathbb{R}^{d}$. Actually this is the very case we are interested in, and we will give the right answer in Section 5 below. To do this we study the rigorous form of the limit theorem and analyze the limit process $\mathbf{X}$. We also show the Feller property of $\mathbf{X}$ under additional assumptions.

\section{Limit theorem}

In this section, we will give the limit theorem for the diffusion processes in the previous section. Our assumptions are the following:

(A.1) The measures $\rho_{0}^{(n)}(\xi) \mathrm{d} \xi$ converge vaguely to a Radon measure $\rho(\mathrm{d} \xi)$ on $\mathbb{R}$.

(A.2) The functions $\mu_{0}^{(n)}$ are decomposed as $\mu_{0}^{(n)}(x)=\mu_{1}^{(n)}\left(x^{\prime}\right) \mu_{2}^{(n)}\left(x^{d}\right)$, and $\mu_{1}^{(n)}\left(x^{\prime}\right)$ converge uniformly to 1 and the measures $\mu_{2}^{(n)}(\xi) \mathrm{d} \xi$ converge vaguely to a non-zero Radon measure $m$ on $\mathbb{R}$.

In order to get our limit theorem, we will express the approximating process $\mathbf{X}^{(n)}$ by means of time change. Let $\mathbf{X}^{\prime}=\left(X_{t}^{\prime}, P_{x^{\prime}}^{\prime}\right)$ be the diffusion process on $\mathbb{R}^{d-1}$ associated with the following Dirichlet form $\left(\mathcal{E}^{\prime}, \mathcal{F}^{\prime}\right)$ on $L^{2}\left(\mathbb{R}^{d-1}, \mathrm{~d} x^{\prime}\right)$ :

$$
\begin{aligned}
& \mathcal{E}^{\prime}(u, v)=\frac{1}{2} \int_{\mathbb{R}^{d-1}} \sum_{i, j=1}^{d-1} a^{i j}\left(x^{\prime}\right) \partial_{x^{i}} u\left(x^{\prime}\right) \partial_{x^{j}} v\left(x^{\prime}\right) \mathrm{d} x^{\prime}, \\
& \mathcal{F}^{\prime}=H^{1}\left(\mathbb{R}^{d-1}\right) .
\end{aligned}
$$

We note that the transition operator $\left\{p_{t}^{\prime}, t>0\right\}$ of $\mathbf{X}^{\prime}$ has the Feller property as in Proposition 2.1. Also let $\Xi=\left(\xi_{t}, P_{\xi}^{\Xi}\right)$ be the one-dimensional diffusion process associated with the following Dirichlet form $\left(\mathcal{E}^{\Xi}, \mathcal{F}^{\Xi}\right)$ on $L^{2}(\mathbb{R}$, d $\xi)$ : 


$$
\mathcal{E}^{\Xi}(u, v)=\frac{1}{2} \int_{\mathbb{R}} u^{\prime}(\xi) v^{\prime}(\xi) a^{d d}(\xi) \mathrm{d} \xi, \quad \mathcal{F}^{\Xi}=H^{1}(\mathbb{R})
$$

In the following the sample paths $X_{t}^{\prime}$, $\xi_{t}$, etc. are sometimes denoted by $X^{\prime}(t), \xi(t)$, etc. It is known that the diffusion process $\Xi$ has the local time $\ell^{\Xi}(t, \xi)$ which is continuous with respect to $(t, \xi) \in[0, \infty) \times \mathbb{R}$ and satisfies $2 \int_{E} \ell^{\Xi}(t, \xi) \mathrm{d} \xi=\int_{0}^{t} I_{E}\left(\xi_{s}\right) \mathrm{d} s, t>0$, for every measurable set $E \subset \mathbb{R}([9])$, where $I_{E}$ is the indicator for a set $E$. We set

$$
\begin{aligned}
\mathbf{f}^{(n)}(t) & =\int_{0}^{t}\left\{1+\rho_{0}^{(n)}\left(\xi_{s}\right)\right\} \mathrm{d} s=t+2 \int_{\mathbb{R}} \ell^{\Xi}(t, \xi) \rho_{0}^{(n)}(\xi) \mathrm{d} \xi, \quad t \geqslant 0, \\
\mathbf{f}(t) & =t+2 \int_{\mathbb{R}} \ell^{\Xi}(t, \xi) \rho(\mathrm{d} \xi), \quad t \geqslant 0, \\
A^{(n)}(t) & =\int_{0}^{t} \mu_{1}^{(n)}\left(X^{\prime}\left(\mathbf{f}^{(n)}(s)\right)\right) \mu_{2}^{(n)}\left(\xi_{s}\right) \mathrm{d} s, \quad t \geqslant 0, \\
A(t) & =2 \int_{\mathbb{R}} \ell^{\Xi}(t, \xi) m(\mathrm{~d} \xi), \quad t \geqslant 0 .
\end{aligned}
$$

Denote the right continuous inverses of $A^{(n)}(t)$ and $A(t)$ by $\tau_{t}^{(n)}$ and $\tau_{t}$ respectively.

Let us consider the time changed process $\widetilde{\mathbf{X}}^{(n)}$ defined by

$$
\widetilde{\mathbf{X}}^{(n)}=\left[\left(X^{\prime}\left(\mathbf{f}^{(n)}\left(\tau_{t}^{(n)}\right)\right), \xi\left(\tau_{t}^{(n)}\right)\right), P_{x}=P_{x^{\prime}}^{\prime} \otimes P_{x^{d}}^{\Xi}, x=\left(x^{\prime}, x^{d}\right) \in \mathbb{R}^{d}\right] .
$$

LEMMA 3.1. - $\widetilde{\mathbf{X}}^{(n)}$ is equivalent to the diffusion process $\mathbf{X}^{(n)}$ defined in Section 2.

Proof. - Since $P_{\xi}^{\Xi}\left(\lim _{t \rightarrow \infty} \mathbf{f}^{(n)}(t)=\infty\right)=1, \xi \in \mathbb{R}$, by virtue of [6], the Dirichlet form of the skew product $\mathbf{Y}^{(n)}:=\left[\left(X^{\prime}\left(\mathbf{f}^{(n)}(t)\right), \xi(t)\right), P_{x}=P_{x^{\prime}}^{\prime} \otimes P_{x^{d}}^{\Xi}, x=\left(x^{\prime}, x^{d}\right) \in \mathbb{R}^{d}\right]$ is given by $\left(\mathcal{E}^{(n)}, \mathcal{F}^{(n)}\right)$, where the underlying measure is $\mathrm{d} x . A^{(n)}(t)$ is a positive continuous additive functional of $\mathbf{Y}^{(n)}$ in the strict sense with Revuz measure $\mu^{(n)}$, which has full quasi support. Therefore, in view of Theorem 6.2.1 in [7], the time changed process $\widetilde{\mathbf{X}}^{(n)}$ is a Hunt process on $\mathbb{R}^{d}$ and the Dirichlet form coincides with $\left(\mathcal{E}^{(n)}, \mathcal{F}^{(n)}\right)$ defined by (2.1), (2.2) with the underlying measure $\mu^{(n)}$. Thus we get the conclusion.

Let $\mathbf{X}$ be the time changed process defined by

$$
\mathbf{X}=\left[\left(X^{\prime}\left(\mathbf{f}\left(\tau_{t}\right)\right), \xi\left(\tau_{t}\right)\right), P_{x}=P_{x^{\prime}}^{\prime} \otimes P_{x^{d}}^{\Xi}, x=\left(x^{\prime}, x^{d}\right) \in \mathbb{R}^{d}\right]
$$

Now our limit theorem is

THEOREM 3.2. - The diffusion processes $\widetilde{\mathbf{X}}^{(n)}$ converge to $\mathbf{X}$ in the sense that for the transition operators $p_{t}^{(n)}$,

$$
\lim _{n \rightarrow \infty} p_{t}^{(n)} f(x)=E_{x}\left[f\left(X^{\prime}\left(\mathbf{f}\left(\tau_{t}\right)\right), \xi\left(\tau_{t}\right)\right)\right], \quad t>0, x \in \mathbb{R}^{d}, f \in C\left(\mathbb{R}^{d}\right),
$$

where $E_{x}$ stands for the expectation with respect to $P_{x}$. 
Proof. - First we note that the set $E_{t}:=\left[\min _{0 \leqslant s \leqslant t} \xi_{s}, \max _{0 \leqslant s \leqslant t} \xi_{s}\right]$ is compact and $\ell^{\Xi}(t, \xi)=0$ for $\xi \notin E_{t}, P_{\xi}^{\Xi}$-a.s., $\xi \in \mathbb{R}$. Hence by means of (A.1) and (A.2),

$$
\begin{gathered}
\lim _{n \rightarrow \infty} \mathbf{f}^{(n)}(t)=\mathbf{f}(t), t \geqslant 0, \quad P_{\xi}^{\Xi} \text {-a.s., } \quad \xi \in \mathbb{R}, \\
\lim _{n \rightarrow \infty} A^{(n)}(t)=A(t), t \geqslant 0, \quad P_{x} \text {-a.s., } x \in \mathbb{R}^{d} .
\end{gathered}
$$

By virtue of (3.11), it holds that

$$
\lim _{n \rightarrow \infty} \tau_{t}^{(n)}=\tau_{t} \quad \text { for } t>0 \text { with } \tau_{t-}=\tau_{t}, \quad P_{x} \text {-a.s. } x \in \mathbb{R}^{d} .
$$

We should notice that

$$
P_{x}\left(\lim _{n \rightarrow \infty} \tau_{t}^{(n)}=\tau_{t}\right)=1, \quad t>0, x \in \mathbb{R}^{d} .
$$

To this end, it is enough to show

$$
P_{\xi}^{\Xi}\left(\tau_{t-}<\tau_{t}\right)=0, \quad t>0, \xi \in \mathbb{R} .
$$

In the case where $\operatorname{supp}[m]=\mathbb{R}, \tau_{t}$ is continuous and hence (3.13) is obvious. Let us consider the case where $S_{m}:=\operatorname{supp}[m] \neq \mathbb{R}$. Then $\mathbb{R} \backslash S_{m}$ is denoted by a finite or a countable disjoint union of open intervals $I_{k}=\left(a_{k}, b_{k}\right), k \in K$ with the end points belonging to $S_{m} \cup\{-\infty, \infty\}$. Since at least one of $a_{k}, b_{k}$ belongs to $S_{m}$ and $\tau_{t-}$ is a Markov time of $\Xi$, we find that for $\varepsilon>0$ and $\xi \in \mathbb{R}$,

$$
\begin{aligned}
P_{\xi}^{\Xi}\left(\tau_{t}-\tau_{t-}>\varepsilon\right) & \leqslant \sum_{k \in K} P_{\xi}^{\Xi}\left(\xi\left(\tau_{t-}\right) \in\left\{a_{k}, b_{k}\right\} \cap S_{m}, \xi_{u} \in I_{k} \text { for } \tau_{t-}<{ }^{\forall} u<\tau_{t-}+\varepsilon\right) \\
& =\sum_{k \in K} E_{\xi}^{\Xi}\left[P_{\xi\left(\tau_{t-}\right)}^{\Xi}\left(\xi_{u} \in I_{k} \text { for } 0<{ }^{\forall} u<\varepsilon\right) ; \xi\left(\tau_{t-}\right) \in\left\{a_{k}, b_{k}\right\} \cap S_{m}\right] .
\end{aligned}
$$

The probability in the last term is zero because of $P_{\xi}^{\Xi}\left(\xi_{u}<\xi\right.$ for $\left.0<{ }^{\forall} u<\varepsilon\right)=P_{\xi}^{\Xi}\left(\xi_{u}>\right.$ $\xi$ for $\left.0<{ }^{\forall} u<\varepsilon\right)=0, \xi \in \mathbb{R}, \varepsilon>0$. Thus we obtain (3.13).

We next note that

$$
P_{x}\left(\lim _{n \rightarrow \infty} \mathbf{f}^{(n)}\left(\tau_{t}^{(n)}\right)=\mathbf{f}\left(\tau_{t}\right)\right)=1, \quad x \in \mathbb{R}^{d}, t>0 .
$$

Indeed,

$$
\begin{aligned}
\left|\mathbf{f}^{(n)}\left(\tau_{t}^{(n)}\right)-\mathbf{f}\left(\tau_{t}\right)\right| \leqslant & \left|\tau_{t}^{(n)}-\tau_{t}\right|+2 \int_{\mathbb{R}}\left|\ell^{\Xi}\left(\tau_{t}^{(n)}, \xi\right)-\ell^{\Xi}\left(\tau_{t}, \xi\right)\right| \rho_{0}^{(n)}(\xi) \mathrm{d} \xi \\
& +2\left|\int_{\mathbb{R}} \ell^{\Xi}\left(\tau_{t}, \xi\right) \rho_{0}^{(n)}(\xi) \mathrm{d} \xi-\int_{\mathbb{R}} \ell^{\Xi}\left(\tau_{t}, \xi\right) \rho(\mathrm{d} \xi)\right| \\
\equiv & I+I I+I I I .
\end{aligned}
$$


By virtue of (3.12), there is an $n_{o} \in \mathbb{N}$ such that $\tau_{t}^{(n)} \leqslant \tau_{t}+1, n \geqslant n_{o}$, and $I \rightarrow 0$ as $n \rightarrow \infty$. Since $\ell^{\Xi}(t, \xi)$ is continuous in $(t, \xi) \in[0, \infty) \times \mathbb{R},(3.12)$ and (A.1) imply that

$$
I I \leqslant 2 \sup _{\xi \in E_{\tau_{t}+1}}\left|\ell^{\Xi}\left(\tau_{t}^{(n)}, \xi\right)-\ell^{\Xi}\left(\tau_{t}, \xi\right)\right| \int_{E_{\tau_{t}+1}} \rho_{0}^{(n)}(\xi) \mathrm{d} \xi \rightarrow 0 \quad \text { as } n \rightarrow \infty .
$$

Since the set $E_{\tau_{t}}$ is compact and $\ell^{\Xi}\left(\tau_{t}, \xi\right)=0$ for $\xi \notin E_{\tau_{t}}$, III $\rightarrow 0$ as $n \rightarrow \infty$. Accordingly (3.14) follows.

By virtue of Lemma 3.1,

$$
p_{t}^{(n)} f(x)=E_{x}\left[f\left(X^{\prime}\left(\mathbf{f}^{(n)}\left(\tau_{t}^{(n)}\right)\right), \xi\left(\tau_{t}^{(n)}\right)\right)\right] .
$$

Combining this with (3.12) and (3.14),

$$
\lim _{n \rightarrow \infty} p_{t}^{(n)} f(x)=E_{x}\left[f\left(X^{\prime}\left(\mathbf{f}\left(\tau_{t}\right)\right), \xi\left(\tau_{t}\right)\right)\right],
$$

because $f \in C\left(\mathbb{R}^{d}\right)$, and $X^{\prime}(t)$ and $\xi(t)$ are continuous in $t$.

Remark 3.3. - By means of (3.12), (3.14) and (3.15), we also see that

$$
\lim _{n \rightarrow \infty} E_{x}\left[\prod_{i=1}^{k} f_{i}\left(X^{\prime}\left(\mathbf{f}^{(n)}\left(\tau_{t_{i}}^{(n)}\right)\right), \xi\left(\tau_{t_{i}}^{(n)}\right)\right)\right]=E_{x}\left[\prod_{i=1}^{k} f_{i}\left(X^{\prime}\left(\mathbf{f}\left(\tau_{t_{i}}\right)\right), \xi\left(\tau_{t_{i}}\right)\right)\right],
$$

for $x \in \mathbb{R}^{d}, 0<t_{1}<t_{2}<\cdots<t_{k}, f_{i} \in C\left(\mathbb{R}^{d}\right)(i=1,2, \ldots, k), k \in \mathbb{N}$, which shows the convergence of finite dimensional distributions of $\widetilde{\mathbf{X}}^{(n)}$ to those of $\mathbf{X}$.

\section{Semigroup of the limit process}

In this section we will show some properties of semigroup of the limit process obtained in the previous section. Let $\mu(\mathrm{d} x)$ be the product measure defined by $\mu(\mathrm{d} x)=$ $\mathrm{d} x^{\prime} \times m\left(\mathrm{~d} x^{d}\right)$, whose support $\Omega_{\mu}$ is given by $\mathbb{R}^{d-1} \times S_{m}$. Since $P_{\xi}^{\Xi}\left(\xi\left(\tau_{t}\right) \in S_{m}, t \geqslant\right.$ $0)=1, \xi \in \mathbb{R}$, it is enough to consider the restrictions of $f$ to $\Omega_{\mu}$ for the right hand side of (3.9). We denote them by $p_{t} f(x)$, that is,

$$
p_{t} f(x)=E_{x}\left[f\left(X^{\prime}\left(\mathbf{f}\left(\tau_{t}\right)\right), \xi\left(\tau_{t}\right)\right)\right], \quad t>0, x \in \mathbb{R}^{d}, f \in C\left(\Omega_{\mu}\right) .
$$

Furthermore we note that $p_{t} f(x)$ is essentially a function of $x \in \Omega_{\mu}$. Let $p^{\prime}\left(t, x^{\prime}, y^{\prime}\right)$ be the transition probability density of the diffusion process $\mathbf{X}^{\prime}$ and $\sigma_{E}^{\Xi}$ the first hitting time of the one-dimensional diffusion process $\Xi$ for a set $E: \sigma_{E}^{\Xi}=\inf \left\{t>0: \xi_{t} \in E\right\}$.

Proposition 4.1. - Let $f \in C\left(\Omega_{\mu}\right), t>0$ and $x=\left(x^{\prime}, x^{d}\right) \in \mathbb{R}^{d} \backslash \Omega_{\mu} \neq \emptyset$. Then

$$
\begin{aligned}
p_{t} f(x)= & \int_{\left(y^{\prime}, y^{d}, s\right) \in \mathbb{R}^{d-1} \times \partial S_{m} \times(0, \infty)} p_{t} f\left(y^{\prime}, y^{d}\right) p^{\prime}\left(s, x^{\prime}, y^{\prime}\right) \mathrm{d} y^{\prime} \\
& \times P_{x^{d}}^{\Xi}\left(\mathbf{f}\left(\sigma_{S_{m}}^{\Xi}\right) \in \mathrm{d} s, \xi\left(\sigma_{S_{m}}^{\Xi}\right) \in \mathrm{d} y^{d}\right),
\end{aligned}
$$

where $\partial S_{m}$ stands for the boundary of $S_{m}$. 
Proof. - Let $\mathbf{Y}=\left(Y_{t}, P_{x}\right)$ be the skew product of $\mathbf{X}^{\prime}$ and $\Xi$ with respect to $\mathbf{f}(t)$ defined by (3.4), that is,

$$
\mathbf{Y}=\left[Y_{t}=\left(X^{\prime}(\mathbf{f}(t)), \xi(t)\right), P_{x}=P_{x^{\prime}}^{\prime} \otimes P_{x^{d}}^{\Xi}, x=\left(x^{\prime}, x^{d}\right) \in \mathbb{R}^{d}\right] .
$$

We note that $\mathbf{Y}$ is a diffusion process on $\mathbb{R}^{d}$. Since $\tau_{t}$ is a Markov time of $\mathbf{Y}$ and $\tau_{s+t}(\omega)=\tau_{s}(\omega)+\tau_{t}\left(\omega_{\tau_{s}(\omega)}^{+}\right)$for $0 \leqslant s \leqslant t$, where $\omega^{+}$stands for the shifted path of $\omega$, by means of the strong Markov property of $\mathbf{Y}$,

$$
E_{x}\left[f\left(Y\left(\tau_{s+t}\right)\right)\right]=E_{x}\left[E_{Y\left(\tau_{s}\right)}\left[f\left(Y\left(\tau_{t}\right)\right)\right]\right], \quad 0 \leqslant s \leqslant t, x \in \mathbb{R}^{d} .
$$

We also note that, for $x \in \mathbb{R}^{d} \backslash \Omega_{\mu}$,

$$
\begin{aligned}
& P_{x}\left(\tau_{0}=\sigma_{S_{m}}^{\Xi}>0\right)=1, \\
& P_{x}\left(Y\left(\tau_{0}\right)=\left(X^{\prime}\left(\mathbf{f}\left(\sigma_{S_{m}}^{\Xi}\right)\right), \xi\left(\sigma_{S_{m}}^{\Xi}\right)\right) \in \mathbb{R}^{d-1} \times \partial S_{m}\right)=1 .
\end{aligned}
$$

Hence putting $s=0$ in (4.4), we get for $x \in \mathbb{R}^{d} \backslash \Omega_{\mu}$,

$$
\begin{aligned}
& E_{x}\left[f\left(X^{\prime}\left(\mathbf{f}\left(\tau_{t}\right)\right), \xi\left(\tau_{t}\right)\right)\right]=E_{x}\left[E_{Y\left(\tau_{0}\right)}\left[f\left(Y\left(\tau_{t}\right)\right)\right]\right] \\
& =\int_{\mathbb{R}^{d-1} \times \partial S_{m}} p_{t} f(y) P_{x}\left(Y\left(\tau_{0}\right) \in \mathrm{d} y\right) \\
& =\int_{\left(y^{\prime}, y^{d}\right) \in \mathbb{R}^{d-1} \times \partial S_{m}} p_{t} f(y) P_{x}\left(X^{\prime}\left(\mathbf{f}\left(\sigma_{S_{m}}^{\Xi}\right)\right) \in \mathrm{d} y^{\prime}, \xi\left(\sigma_{S_{m}}^{\Xi}\right) \in \mathrm{d} y^{d}\right) \\
& =\int_{\left(y^{\prime}, y^{d}, s\right) \in \mathbb{R}^{d-1} \times \partial S_{m} \times(0, \infty)} p_{t} f\left(y^{\prime}, y^{d}\right) P_{x^{\prime}}^{\prime}\left(X_{s}^{\prime} \in \mathrm{d} y^{\prime}\right) \\
& \quad \times P_{x^{d}}^{\Xi}\left(\mathbf{f}\left(\sigma_{S_{m}}^{\Xi}\right) \in \mathrm{d} s, \xi\left(\sigma_{S_{m}}^{\Xi}\right) \in \mathrm{d} y^{d}\right) \\
& =\quad p_{\left(y^{\prime}, y^{d}, s\right) \in \mathbb{R}^{d-1} \times \partial S_{m} \times(0, \infty)} p_{t} f\left(y^{\prime}, y^{d}\right) p^{\prime}\left(s, x^{\prime}, y^{\prime}\right) \mathrm{d} y^{\prime} \\
& \quad \times P_{x^{d}}^{\Xi}\left(\mathbf{f}\left(\sigma_{S_{m}}^{\Xi}\right) \in \mathrm{d} s, \xi\left(\sigma_{S_{m}}^{\Xi}\right) \in \mathrm{d} y^{d}\right) .
\end{aligned}
$$

This shows (4.2).

Remark 4.2. - If $\mathbb{R} \backslash S_{m} \neq \emptyset$, then $\mathbb{R} \backslash S_{m}$ is expressed by a finite or a countable disjoint union of open intervals $I_{k}$ with the end points belonging to $S_{m} \cup\{-\infty, \infty\}$. Suppose that $x^{d} \in I_{k} \equiv(a, b)$. Then

$$
\sigma_{S_{m}}^{\Xi}=\left\{\begin{array}{ll}
\sigma_{b}^{\Xi}, & -\infty=a<b<\infty, \\
\min \left\{\sigma_{a}^{\Xi}, \sigma_{b}^{\Xi}\right\}, & -\infty<a<b<\infty, \\
\sigma_{a}^{\Xi}, & -\infty<a<b=\infty,
\end{array} \quad P_{x^{d}}^{\Xi}\right. \text {-a.s. }
$$

where $\sigma_{p}^{\Xi}$ is the first hitting time to a point $p$. Hence

$$
P_{x^{d}}^{\Xi}\left(\mathbf{f}\left(\sigma_{S_{m}}^{\Xi}\right) \in \mathrm{d} s, \xi\left(\sigma_{S_{m}}^{\Xi}\right) \in \mathrm{d} y^{d}\right)
$$




$$
= \begin{cases}P_{x^{d}}^{\Xi}\left(\mathbf{f}\left(\sigma_{b}^{\Xi}\right) \in \mathrm{d} s\right) \delta_{b}\left(\mathrm{~d} y^{d}\right), & -\infty=a<b<\infty, \\ P_{x^{d}}^{\Xi}\left(\mathbf{f}\left(\sigma_{a}^{\Xi}\right) \in \mathrm{d} s, \sigma_{a}^{\Xi}<\sigma_{b}^{\Xi}\right) \delta_{a}\left(\mathrm{~d} y^{d}\right) & -\infty<a<b<\infty, \\ \quad+P_{x^{d}}^{\Xi}\left(\mathbf{f}\left(\sigma_{b}^{\Xi}\right) \in \mathrm{d} s, \sigma_{b}^{\Xi}<\sigma_{a}^{\Xi}\right) \delta_{b}\left(\mathrm{~d} y^{d}\right), & \\ P_{x^{d}}^{\Xi}\left(\mathbf{f}\left(\sigma_{a}^{\Xi}\right) \in \mathrm{d} s\right) \delta_{a}\left(\mathrm{~d} y^{d}\right), & -\infty<a<b=\infty,\end{cases}
$$

where $\delta_{p}(\mathrm{~d} \xi)$ is the unit measure concentrated at a point $p$.

We next show that $\left\{p_{t}, t>0\right\}$ is a Feller semigroup. Put $l_{1}=\inf S_{m}, l_{2}=\sup S_{m}$.

THEOREM 4.3. - Let us assume that

$$
\begin{aligned}
& \int_{-\infty}^{0}|\xi| m(\mathrm{~d} \xi)=\infty \quad \text { if } l_{1}=-\infty \\
& \int_{0}^{\infty} \xi m(\mathrm{~d} \xi)=\infty \quad \text { if } l_{2}=\infty .
\end{aligned}
$$

Then it holds that

$$
\begin{aligned}
p_{t} f \in C\left(\mathbb{R}^{d}\right) \cap \widehat{C}\left(\Omega_{\mu}\right) & \text { for } t>0, f \in \widehat{C}\left(\Omega_{\mu}\right), \\
\limsup _{t \downarrow 0 x \in \Omega_{\mu}}\left|p_{t} f(x)-f(x)\right|=0 & \text { for } f \in \widehat{C}\left(\Omega_{\mu}\right) .
\end{aligned}
$$

For the proof of Theorem 4.3, we prepare some lemmas.

First of all we give an expression of $p_{t} f(x)$ in terms of the one-dimensional Brownian motion $\mathbf{B}=\left(B_{t}, P_{\xi}^{B}\right)$. We set

$$
s(\xi)=\int_{0}^{\xi}\left\{1 / a^{d d}(\eta)\right\} \mathrm{d} \eta, \quad \xi \in \mathbb{R} .
$$

Obviously $s(\xi)$ is a strictly increasing continuous function on $\mathbb{R}$ and $s(-\infty)=$ $-\infty, s(\infty)=\infty$. Since $\Lambda^{-1} \leqslant a^{d d}(\xi) \leqslant \Lambda, \xi \in \mathbb{R}$, for some positive constant $\Lambda>1$, we see that

$$
\Lambda^{-1}|\xi| \leqslant|s(\xi)| \leqslant \Lambda|\xi|, \quad \xi \in \mathbb{R} .
$$

Denote by $\gamma(\xi)$ the inverse of $s(\xi)$. Note that $\gamma^{\prime}(\xi)=a^{d d}(\gamma(\xi))$, a.e. $\xi \in \mathbb{R}$, and

$$
\Lambda^{-1}|\xi| \leqslant|\gamma(\xi)| \leqslant \Lambda|\xi|, \quad \xi \in \mathbb{R} .
$$

For each $a \in \mathbb{R}$, we set

$$
\Phi(t ; a)=2 \int_{\mathbb{R}} \ell^{B}(t, s(\xi)-s(a)) \mathrm{d} \xi, \quad t \geqslant 0,
$$

where $\ell^{B}(t, \xi)$ is the local time of the Brownian motion $\mathbf{B}$, that is, $\int_{0}^{t} I_{E}\left(B_{s}\right) \mathrm{d} s=$ $2 \int_{E} \ell^{B}(t, \xi) \mathrm{d} \xi$, for measurable sets $E \subset \mathbb{R}$. We denote by $\phi(t ; a)$ the inverse of 
$t \longmapsto \Phi(t ; a) . \phi(t ; a)$ is a strictly increasing continuous function in $t$. We note that

$$
\Phi(t ; a)=2 \int_{\mathbb{R}} \ell^{B}(t, \eta) \gamma^{\prime}(\eta+s(a)) \mathrm{d} \eta, \quad t \geqslant 0 .
$$

Therefore

$$
t / \Lambda \leqslant \Phi(t ; a) \leqslant \Lambda t, \quad t \geqslant 0, a \in \mathbb{R}
$$

from which

$$
t / \Lambda \leqslant \phi(t ; a) \leqslant \Lambda t, \quad t \geqslant 0, a \in \mathbb{R}
$$

Further we put

$$
\begin{aligned}
\mathbf{f}(t ; a) & =t+2 \int_{\mathbb{R}} \ell^{B}(\phi(t ; a), s(\xi)-s(a)) \rho(\mathrm{d} \xi), \\
A(t ; a) & =2 \int_{\mathbb{R}} \ell^{B}(\phi(t ; a), s(\xi)-s(a)) m(\mathrm{~d} \xi), \\
\tau(t ; a) & =\text { the right continuous inverse of } t \longmapsto A(t ; a) .
\end{aligned}
$$

LEMMA 4.4. - It holds that for every $t>0, x \in \mathbb{R}^{d}, f \in C\left(\Omega_{\mu}\right)$,

$$
p_{t} f(x)=E_{0}^{B}\left[E_{x^{\prime}}^{\prime}\left[f\left(X_{\mathbf{f}\left(\tau\left(t ; x^{d}\right) ; x^{d}\right)}^{\prime}, \gamma\left(B_{\phi\left(\tau\left(t ; x^{d}\right) ; x^{d}\right)}+s\left(x^{d}\right)\right)\right)\right]\right],
$$

where $E_{x^{\prime}}^{\prime}, E_{x^{d}}^{B}$ stand for the expectations with respect to $P_{x^{\prime}}^{\prime}, P_{x^{d}}^{B}$ respectively, and the right hand side of (4.12) should be read as

$$
E_{0}^{B}\left[\left.E_{x^{\prime}}^{\prime}\left[f\left(X_{u}^{\prime}, \xi\right)\right]\right|_{\left.u=\mathbf{f}\left(\tau\left(t ; x^{d}\right) ; x^{d}\right), \xi=\gamma\left(B_{\phi\left(\tau\left(t ; x^{d}\right) ; x^{d}\right)}+s\left(x^{d}\right)\right)\right] .}\right.
$$

Proof. - Following the terminology in [9], $\Xi$ is a diffusion process on $\mathbb{R}$ with the scale function $s(\xi)$ and the speed measure $2 \mathrm{~d} \xi$. By virtue of argument in [9, Ch. 5], $\Xi=\left(\xi_{t}, P_{\xi}^{\Xi}\right)$ is equivalent to the time changed process $\left(\gamma\left(B_{\phi_{t}}\right), P_{s(\xi)}^{B}\right)$, where

$$
\begin{aligned}
\Phi(t) & =2 \int_{\mathbb{R}} \ell^{B}(t, \xi) \gamma^{\prime}(\xi) \mathrm{d} \xi=2 \int_{\mathbb{R}} \ell^{B}(t, s(\xi)) \mathrm{d} \xi, \\
\phi_{t} & =\text { the inverse of } t \longmapsto \Phi(t) .
\end{aligned}
$$

Since $\left(B_{t}, P_{s(\xi)}^{B}\right)$ is also equivalent to $\left(B_{t}+s(\xi), P_{0}^{B}\right)$, the process $\left(\ell^{\Xi}(t, \cdot), P_{\xi}^{\Xi}\right)$ is equivalent to $\left(\ell^{B}(\phi(t ; \xi), s(\cdot)-s(\xi)), P_{0}^{B}\right)$ and $\left(\xi_{t}, P_{\xi}^{\Xi}\right)$ is equivalent to $\left(\gamma\left(B_{\phi(t ; \xi)}+\right.\right.$ $\left.s(\xi)), P_{0}^{B}\right)$. Combining these facts with (4.1) and using of Fubini's theorem, we get the assertion of the lemma.

Remark 4.5. - It should be noted that $D:=\left(\gamma\left(B_{\phi(\tau(t ; \xi) ; \xi)}+s(\xi)\right), P_{0}^{B}\right)$ is a onedimensional generalized diffusion process having the scale function $s$ and the speed measure $2 m$ (cf. [9]). $D$ is conservative because $m$ is a Radon measure on $\mathbb{R}$. When $l_{1}>-\infty\left[l_{2}<\infty\right]$, the boundary $l_{1}\left[l_{2}\right]$ is regular reflecting in the sense of Feller [5]. When $l_{1}=-\infty\left[l_{2}=\infty\right]$, the boundary $l_{1}\left[l_{2}\right]$ is entrance or natural in the sense 
of Feller [5] according as the integral $\int_{-\infty}^{0}|s(\xi)| m(\mathrm{~d} \xi)\left[\int_{0}^{\infty} s(\xi) m(\mathrm{~d} \xi)\right]$ converges or diverges. The transition operator $\left\{p_{t}^{D}\right\}$ possesses the following properties:

$$
\begin{aligned}
& p_{t}^{D}\left(B\left(S_{m}\right)\right) \subset C\left(S_{m}\right), \\
& \lim _{\xi \rightarrow l_{i}, \xi \in S_{m}} p_{t}^{D} f(\xi)=0 \quad \text { if } l_{i} \text { is natural and } \lim _{\xi \rightarrow l_{i}, \xi \in S_{m}} f(\xi)=0, \quad \text { for each } i=1,2, \\
& \limsup _{t \downarrow 0}\left|p_{t}^{D} f(\xi)-f(\xi)\right|=0, \quad f \in \widehat{C}\left(S_{m}\right),
\end{aligned}
$$

where $B\left(S_{m}\right)$ stands for the space of all bounded measurable functions on $S_{m}$. In the case where $l_{1}\left[l_{2}\right]$ is entrance, it happens that $\lim _{\xi \rightarrow l_{1}\left[l_{2}\right], \xi \in S_{m}} p_{t}^{D} f(\xi) \neq 0$ even if $\lim _{\xi \rightarrow l_{1}\left[l_{2}\right], \xi \in S_{m}} f(\xi)=0$.

LemmA 4.6. - Let $\left\{a_{n}\right\}$ be a sequence converging to $a \in \mathbb{R}$. Then

$$
\begin{aligned}
& P_{0}^{B}\left(\lim _{n \rightarrow \infty} \Phi\left(t ; a_{n}\right)=\Phi(t ; a), t \geqslant 0\right)=1, \\
& P_{0}^{B}\left(\lim _{n \rightarrow \infty} \phi\left(t ; a_{n}\right)=\phi(t ; a), t \geqslant 0\right)=1, \\
& P_{0}^{B}\left(\lim _{n \rightarrow \infty} \mathbf{f}\left(t ; a_{n}\right)=\mathbf{f}(t ; a), t \geqslant 0\right)=1, \\
& P_{0}^{B}\left(\lim _{n \rightarrow \infty} A\left(t ; a_{n}\right)=A(t ; a), t \geqslant 0\right)=1, \\
& P_{0}^{B}\left(\lim _{n \rightarrow \infty} \tau\left(t ; a_{n}\right)=\tau(t ; a)\right)=1, \quad t>0, \\
& P_{0}^{B}\left(\lim _{n \rightarrow \infty} \phi\left(\tau\left(t ; a_{n}\right) ; a_{n}\right)=\phi(\tau(t ; a) ; a)\right)=1, \quad t>0, \\
& P_{0}^{B}\left(\lim _{n \rightarrow \infty} \mathbf{f}\left(\tau\left(t ; a_{n}\right) ; a_{n}\right)=\mathbf{f}(\tau(t ; a) ; a)\right)=1, \quad t>0 .
\end{aligned}
$$

Proof. - We set $\gamma_{a}(\mathrm{~d} \eta)=\gamma^{\prime}(\eta+s(a)) \mathrm{d} \eta$. The Radon measures $\gamma_{a_{n}}$ converge to the Radon measure $\gamma_{a}$ vaguely as $a_{n} \rightarrow a$. Therefore (4.16) follows from (4.10) by the same reason as for (3.10). (4.17) is a direct consequence of (4.16). We set $\widetilde{\rho}(\xi)=$ $\rho([0, \xi]), \quad \xi \geqslant 0,=-\rho((\xi, 0)), \quad \xi<0$, and further $\widetilde{\rho}_{a}(\xi)=\widetilde{\rho}(\gamma(\xi+s(a)))$. Then it holds that

$$
\mathbf{f}(t ; a)=t+2 \int_{\mathbb{R}} \ell^{B}(\phi(t ; a), \xi) \widetilde{\rho}_{a}(\mathrm{~d} \xi) .
$$

Since the measures $\widetilde{\rho}_{a_{n}}$ converge to the measure $\widetilde{\rho}_{a}$ vaguely as $a_{n} \rightarrow a$, we get (4.18) by the same argument as for (3.14). In a similar way we obtain (4.19). We can derive (4.20) from (4.19) by the same argument as for (3.12). It is easy to see that

$$
\begin{aligned}
\lim _{n \rightarrow \infty} A\left(\Phi\left(t ; a_{n}\right) ; a_{n}\right) & =\lim _{n \rightarrow \infty} 2 \int_{\mathbb{R}} \ell^{B}\left(t, s(\xi)-s\left(a_{n}\right)\right) m(\mathrm{~d} \xi) \\
& =2 \int_{\mathbb{R}} \ell^{B}(t, s(\xi)-s(a)) m(\mathrm{~d} \xi) \\
& =A(\Phi(t ; a) ; a), \quad t \geqslant 0, \quad P_{0}^{B} \text {-a.s. }
\end{aligned}
$$


Since $\phi(\tau(t ; a) ; a)$ is the right continuous inverse of $t \longmapsto A(\Phi(t ; a) ; a)$, we get (4.21) by the same reason as for (3.12). We obtain (4.22) from (4.18) and (4.20) by the same method as for (3.14).

Proposition 4.7. - It holds that

$$
p_{t}\left(\widehat{C}\left(\Omega_{\mu}\right)\right) \subset C\left(\mathbb{R}^{d}\right), \quad t>0 .
$$

Proof. - Let us fix $t>0$ and $f \in \widehat{C}\left(\Omega_{\mu}\right)$ arbitrarily and let $\left\{x_{n}\right\}_{n=1}^{\infty}$ be a sequence converging to $x_{0} \in \mathbb{R}^{d}$. We set

$$
u_{n}=\mathbf{f}\left(\tau\left(t ; x_{n}^{d}\right) ; x_{n}^{d}\right), \quad \xi_{n}=\gamma\left(B_{\phi\left(\tau\left(t ; x_{n}^{d}\right) ; x_{n}^{d}\right)}+s\left(x_{n}^{d}\right)\right), \quad n \in \mathbb{N} \cup\{0\} .
$$

By means of Lemma 4.4,

$$
\begin{aligned}
\left|p_{t} f\left(x_{n}\right)-p_{t} f\left(x_{0}\right)\right| \leqslant & E_{0}^{B}\left[\left|E_{x_{n}^{\prime}}^{\prime}\left[f\left(X^{\prime}\left(u_{n}\right), \xi_{n}\right)\right]-E_{x_{0}^{\prime}}^{\prime}\left[f\left(X^{\prime}\left(u_{0}\right), \xi_{0}\right)\right]\right|\right] \\
\leqslant & E_{0}^{B}\left[E_{x_{n}^{\prime}}^{\prime}\left[\left|f\left(X^{\prime}\left(u_{n}\right), \xi_{n}\right)-f\left(X^{\prime}\left(u_{n}\right), \xi_{0}\right)\right|\right]\right] \\
& +E_{0}^{B}\left[\left|E_{x_{n}^{\prime}}^{\prime}\left[f\left(X^{\prime}\left(u_{n}\right), \xi_{0}\right)\right]-E_{x_{0}^{\prime}}^{\prime}\left[f\left(X^{\prime}\left(u_{0}\right), \xi_{0}\right)\right]\right|\right] \\
\leqslant & E_{0}^{B}\left[\sup _{y^{\prime} \in \mathbb{R}^{d-1}}\left|f\left(y^{\prime}, \xi_{n}\right)-f\left(y^{\prime}, \xi_{0}\right)\right|\right] \\
& +E_{0}^{B}\left[\left|p_{u_{n}}^{\prime} f\left(\cdot, \xi_{0}\right)\left(x_{n}^{\prime}\right)-p_{u_{0}}^{\prime} f\left(\cdot, \xi_{0}\right)\left(x_{0}^{\prime}\right)\right|\right] \\
\equiv & I_{n}+I I_{n} .
\end{aligned}
$$

Since $P_{0}^{B}\left(\lim _{n \rightarrow \infty} \xi_{n}=\xi_{0}\right)=1$ by virtue of Lemma 4.6,

$$
\limsup _{n \rightarrow \infty} I_{n} \leqslant E_{0}^{B}\left[\limsup _{n \rightarrow \infty} \sup _{y^{\prime} \in \mathbb{R}^{d-1}}\left|f\left(y^{\prime}, \xi_{n}\right)-f\left(y^{\prime}, \xi_{0}\right)\right|\right]=0 .
$$

Noting that $\left\{p_{t}^{\prime}, t>0\right\}$ is a Feller semigroup, and $P_{0}^{B}\left(\lim _{n \rightarrow \infty} u_{n}=u_{0}\right)=1$ by Lemma 4.6, we have

$$
\limsup _{n \rightarrow \infty} I_{n} \leqslant E_{0}^{B}\left[\limsup _{n \rightarrow \infty}\left|p_{u_{n}}^{\prime} f\left(\cdot, \xi_{0}\right)\left(x_{n}^{\prime}\right)-p_{u_{0}}^{\prime} f\left(\cdot, \xi_{0}\right)\left(x_{0}^{\prime}\right)\right|\right]=0 .
$$

We thus get the conclusion of the proposition.

LEMMA 4.8. - For each $K>0$, let

$$
\mathbf{f}_{K}(t)=\sup _{|a| \leqslant K} \mathbf{f}(t ; a), \quad A_{K}(t)=\inf _{|a| \leqslant K, a \in S_{m}} A(t ; a) .
$$

Then it holds that

$$
\begin{aligned}
& P_{0}^{B}\left(\mathbf{f}_{K}(0+)=0\right)=1, \\
& P_{0}^{B}\left(\mathbf{f}_{K}(t)<\infty, t \geqslant 0\right)=1, \\
& P_{0}^{B}\left(A_{K}(t)>0, t>0\right)=1 \quad \text { if } S_{m} \cap[-K, K] \neq \emptyset \\
& P_{0}^{B}\left(\lim _{t \rightarrow \infty} A_{K}(t)=\infty\right)=1 .
\end{aligned}
$$


Proof. - Let $a \in \mathbb{R},-\infty<\alpha \leqslant 0 \leqslant \beta<\infty$ and $I=[\gamma(s(a)+\alpha), \gamma(s(a)+\beta)]$. We then note that

$$
\begin{aligned}
& s(\xi)-s(a) \in[\alpha, \beta] \quad \text { if and only if } \quad \xi \in I, \\
& {\left[a+\Lambda^{-1} \alpha, a+\Lambda^{-1} \beta\right] \subset I \subset[a+\Lambda \alpha, a+\Lambda \beta] .}
\end{aligned}
$$

Indeed,

$$
\gamma(s(a)+\beta)-a=\gamma(s(a)+\beta)-\gamma(s(a))=\int_{s(a)}^{s(a)+\beta} \gamma^{\prime}(\eta) \mathrm{d} \eta,
$$

and hence $a+\Lambda^{-1} \beta \leqslant \gamma(s(a)+\beta) \leqslant a+\Lambda \beta$. In the same way, $a+\Lambda \alpha \leqslant \gamma(s(a)+\alpha) \leqslant$ $a+\Lambda^{-1} \alpha$. Therefore we get (4.28).

We set $\alpha_{t}=\min _{0 \leqslant s \leqslant t} B_{s}, \beta_{t}=\max _{0 \leqslant s \leqslant t} B_{s}$, and $E_{t}=\left[\alpha_{t}, \beta_{t}\right]$. By means of (4.11), (4.27) and (4.28),

$$
\begin{aligned}
\int_{\mathbb{R}} \ell^{B}(\phi(t ; a), s(\xi)-s(a)) \rho(\mathrm{d} \xi) & \leqslant \int_{\mathbb{R}} \ell^{B}(\Lambda t, s(\xi)-s(a)) \rho(\mathrm{d} \xi) \\
& \leqslant \max _{\eta \in E_{\Lambda t}} \ell^{B}(\Lambda t, \eta) \rho\left(\left\{\xi: s(\xi)-s(a) \in E_{\Lambda t}\right\}\right) \\
& \leqslant \max _{\eta \in E_{\Lambda t}} \ell^{B}(\Lambda t, \eta) \rho\left(\left[a+\Lambda \alpha_{\Lambda t}, a+\Lambda \beta_{\Lambda t}\right]\right) \\
& \leqslant \max _{\eta \in E_{\Lambda t}} \ell^{B}(\Lambda t, \eta) \rho\left(\left[-K+\Lambda \alpha_{\Lambda t}, K+\Lambda \beta_{\Lambda t}\right]\right),
\end{aligned}
$$

from which (4.23) follows.

By means of (4.18), $\mathbf{f}(t, a)$ is continuous in $a \in \mathbb{R}$, which implies (4.24).

By using (4.11), (4.27) and (4.28) again, and noting that $\Lambda^{-1}<1$,

$$
\begin{aligned}
A(t ; a) \geqslant & 2 \int_{\xi: s(\xi)-s(a) \in E_{t / \Lambda}} \ell^{B}(t / \Lambda, s(\xi)-s(a)) m(\mathrm{~d} \xi) \\
\geqslant & 2 \int_{\xi: s(\xi)-s(a) \in\left[\Lambda^{-1} \alpha_{t / \Lambda}, \Lambda^{-1} \beta_{t / \Lambda}\right]} \ell^{B}(t / \Lambda, s(\xi)-s(a)) m(\mathrm{~d} \xi) \\
\geqslant & 2 \min _{\eta \in\left[\Lambda^{-1} \alpha_{t / \Lambda}, \Lambda^{-1} \beta_{t / \Lambda}\right]} \ell^{B}(t / \Lambda, \eta) \\
& \times m\left(\left\{\xi: s(\xi)-s(a) \in\left[\Lambda^{-1} \alpha_{t / \Lambda}, \Lambda^{-1} \beta_{t / \Lambda}\right]\right\}\right) \\
\geqslant & 2 \min _{\eta \in\left[\Lambda^{-1} \alpha_{t / \Lambda}, \Lambda^{-1} \beta_{t / \Lambda}\right]} \ell^{B}(t / \Lambda, \eta) m\left(\left[a+\Lambda^{-2} \alpha_{t / \Lambda}, a+\Lambda^{-2} \beta_{t / \Lambda}\right]\right) .
\end{aligned}
$$

Hence

$$
A_{K}(t) \geqslant 2 \min _{\eta \in\left[\Lambda^{-1} \alpha_{t / \Lambda}, \Lambda^{-1} \beta_{t / \Lambda}\right]} \ell^{B}(t / \Lambda, \eta) \inf _{|a| \leqslant K, a \in S_{m}} m\left(\left[a+\Lambda^{-2} \alpha_{t / \Lambda}, a+\Lambda^{-2} \beta_{t / \Lambda}\right]\right) .
$$

We notice that

$$
\min _{\eta \in\left[\Lambda^{-1} \alpha_{t / \Lambda}, \Lambda^{-1} \beta_{t / \Lambda}\right]} \ell^{B}(t / \Lambda, \eta)>0, \quad t>0
$$


because of $\Lambda^{-1}<1$. We next note that

$$
\inf _{|a| \leqslant K, a \in S_{m}} m\left(\left[a+\Lambda^{-2} \alpha_{t / \Lambda}, a+\Lambda^{-2} \beta_{t / \Lambda}\right]\right)>0, \quad t>0 .
$$

For, if not, there exists an $a$ such that $|a| \leqslant K, a \in S_{m}$ and

$$
m\left(\left(a+\Lambda^{-2} \alpha_{t / \Lambda}, a+\Lambda^{-2} \beta_{t / \Lambda}\right)\right)=0,
$$

which contradicts that $a \in S_{m}$. Thus we get (4.25).

We take an $R>0$ such that $m([-R, R])>0$. Then

$$
\begin{aligned}
A(t ; a) & \geqslant 2 \int_{|\xi| \leqslant R} \ell^{B}(t / \Lambda, s(\xi)-s(a)) m(\mathrm{~d} \xi) \\
& \geqslant 2 \min _{\eta=s(\xi)-s(a),|\xi| \leqslant R} \ell^{B}(t / \Lambda, \eta) m([-R, R]) \\
& \geqslant 2 \min _{|\eta| \leqslant \Lambda(R+K)} \ell^{B}(t / \Lambda, \eta) m([-R, R]) .
\end{aligned}
$$

In order to get (4.26), it is enough to show that

$$
P_{0}^{B}\left(\lim _{t \rightarrow \infty|\xi| \leqslant L} \min ^{B}(t, \xi)=\infty\right)=1, \quad L>0 .
$$

Since $\left(B(t), P_{0}^{B}\right)$ is equivalent to $\left(c^{-1} B\left(c^{2} t\right), P_{0}^{B}\right)$ and hence $\left(\ell^{B}(t, \xi), P_{0}^{B}\right)$ is equivalent to $\left(c^{-1} \ell^{B}\left(c^{2} t, c \xi\right), P_{0}^{B}\right)$ for $c>0$, we see that, for every $r>0$,

$$
\begin{aligned}
P_{0}^{B}\left(\lim _{k \rightarrow \infty} \min _{|\xi| \leqslant L} \ell^{B}(k, \xi)>r\right) & =P_{0}^{B}\left(\bigcup_{k \in \mathbb{N}}\left\{\min _{|\xi| \leqslant L} \ell^{B}(k, \xi)>r\right\}\right) \\
& =\lim _{k \rightarrow \infty} P_{0}^{B}\left(\sqrt{k} \min _{|\xi| \leqslant L} \ell^{B}(1, \xi / \sqrt{k})>r\right) \\
& =P_{0}^{B}\left(\bigcup_{k \in \mathbb{N}}\left\{\min _{|\xi| \leqslant L / \sqrt{k}} \ell^{B}(1, \xi)>r / \sqrt{k}\right\}\right) \\
& =P_{0}^{B}\left(\ell^{B}(1,0)>0\right)=1 .
\end{aligned}
$$

We denote by $\tau_{K}(t)$ the right continuous inverse of $t \longmapsto A_{K}(t)$. By virtue of Lemma 4.8,

$$
\begin{gathered}
\sup _{|a| \leqslant K, a \in S_{m}} \mathbf{f}(\tau(t ; a) ; a) \leqslant \mathbf{f}_{K}\left(\tau_{K}(t)\right)<\infty, \quad t>0, \quad P_{0}^{B} \text {-a.s. } \\
\mathbf{f}_{K}\left(\tau_{K}(0+)\right)=0, \quad P_{0}^{B} \text {-a.s. }
\end{gathered}
$$

for every $K>0$.

PROPOSITION 4.9. - It holds that

$$
\lim _{\left|x^{\prime}\right| \rightarrow \infty} \sup _{\left|x^{d}\right| \leqslant K, x^{d} \in S_{m}}\left|p_{t} f(x)\right|=0, \quad f \in \widehat{C}\left(\Omega_{\mu}\right), K>0 .
$$


Proof. - We set $h\left(y^{\prime}\right)=\sup _{y^{d} \in \mathbb{R}}\left|f\left(y^{\prime}, y^{d}\right)\right|\left(\in \widehat{C}\left(\mathbb{R}^{d-1}\right)\right)$ and $u=\mathbf{f}\left(\tau\left(t ; x^{d}\right) ; x^{d}\right)$. By virtue of Lemma 4.4,

$$
\left|p_{t} f(x)\right| \leqslant E_{0}^{B}\left[E_{x^{\prime}}^{\prime}\left[h\left(X_{u}^{\prime}\right)\right]\right]=E_{0}^{B}\left[\int_{\mathbb{R}^{d-1}} p^{\prime}\left(u, x^{\prime}, y^{\prime}\right) h\left(y^{\prime}\right) \mathrm{d} y^{\prime}\right] .
$$

It is well known that

$$
\begin{aligned}
& C_{1} t^{-(d-1) / 2} \mathrm{e}^{-C_{2}\left|x^{\prime}-y^{\prime}\right|^{2} / t} \leqslant p^{\prime}\left(t, x^{\prime}, y^{\prime}\right) \leqslant C_{3} t^{-(d-1) / 2} \mathrm{e}^{-C_{4}\left|x^{\prime}-y^{\prime}\right|^{2} / t}, \\
& \quad t>0, x^{\prime}, y^{\prime} \in \mathbb{R}^{d-1},
\end{aligned}
$$

with some positive constants $C_{i}(i=1,2,3,4)([3,4])$, from which

$$
\begin{aligned}
& \limsup _{\left|x^{\prime}\right| \rightarrow \infty} \sup _{\left|x^{d}\right| \leqslant K, x^{d} \in S_{m}}\left|p_{t} f(x)\right| \\
& \leqslant E_{0}^{B}\left[\limsup _{\left|x^{\prime}\right| \rightarrow \infty} \sup _{\left|x^{d}\right| \leqslant K, x^{d} \in S_{m}} \int_{\mathbb{R}^{d-1}} p^{\prime}\left(u, x^{\prime}, y^{\prime}\right) h\left(y^{\prime}\right) \mathrm{d} y^{\prime}\right] \\
& \leqslant C_{3} E_{0}^{B}\left[\limsup _{\left|x^{\prime}\right| \rightarrow \infty} \sup _{\left|x^{d}\right| \leqslant K, x^{d} \in S_{m}} \int_{\mathbb{R}^{d-1}} \mathrm{e}^{-C_{4}\left|y^{\prime}\right|^{2}} h\left(x^{\prime}+\sqrt{u} y^{\prime}\right) \mathrm{d} y^{\prime}\right] \\
& \leqslant C_{3} E_{0}^{B}\left[\int_{\mathbb{R}^{d-1}} \mathrm{e}^{-C_{4}\left|y^{\prime}\right|^{2}} \limsup _{\left|x^{\prime}\right| \rightarrow \infty} \sup _{\left|x^{d}\right| \leqslant K, x^{d} \in S_{m}} h\left(x^{\prime}+\sqrt{u} y^{\prime}\right) \mathrm{d} y^{\prime}\right] .
\end{aligned}
$$

Noting (4.29), we see that

$$
\sup _{\left|x^{\prime}\right| \rightarrow \infty} \sup _{\left|x^{d}\right| \leqslant K, x^{d} \in S_{m}} h\left(x^{\prime}+\sqrt{u} y^{\prime}\right)=0, \quad y^{\prime} \in \mathbb{R}^{d-1} .
$$

We thus obtain the conclusion of the proposition.

Proposition 4.10. - If (4.5) is satisfied, then

$$
\lim _{x^{d} \rightarrow-\infty, x^{d} \in S_{m}} \sup _{x^{\prime} \in \mathbb{R}^{d-1}}\left|p_{t} f(x)\right|=0, \quad f \in \widehat{C}\left(\Omega_{\mu}\right) .
$$

If (4.6) is satisfied, then

$$
\lim _{x^{d} \rightarrow \infty, x^{d} \in S_{m}} \sup _{x^{\prime} \in \mathbb{R}^{d-1}}\left|p_{t} f(x)\right|=0, \quad f \in \widehat{C}\left(\Omega_{\mu}\right) .
$$

Proof. - We set $h\left(y^{d}\right)=\sup _{y^{\prime} \in \mathbb{R}^{d-1}}\left|f\left(y^{\prime}, y^{d}\right)\right|$, which belongs to $\widehat{C}\left(S_{m}\right)$. By virtue of Lemma 4.4 and Remark 4.5,

$$
\sup _{x^{\prime} \in \mathbb{R}^{d-1}}\left|p_{t} f(x)\right| \leqslant E_{0}^{B}\left[h\left(\gamma\left(B_{\phi\left(\tau\left(t ; x^{d}\right) ; x^{d}\right)}+s\left(x^{d}\right)\right)\right)\right]=p_{t}^{D} h\left(x^{d}\right) .
$$

By means of (4.9) and Remark 4.5, the assumption (4.5) [(4.6)] implies that if $l_{1}=$ $-\infty\left[l_{2}=\infty\right]$, it is a natural boundary. Accordingly, (4.32) and (4.33) immediately follow from (4.14). 
Proposition 4.11. - Suppose that (4.5) and (4.6) are satisfied. Then it holds that

$$
\limsup _{t \downarrow 0}\left|p_{t} f(x)-f(x)\right|=0, \quad f \in \widehat{C}\left(\Omega_{\mu}\right) .
$$

Proof. - Let $f \in \widehat{C}\left(\Omega_{\mu}\right)$ and put $h\left(y^{d}\right)=\sup _{y^{\prime} \in \mathbb{R}^{d-1}}\left|f\left(y^{\prime}, y^{d}\right)\right|$. Let us fix an $\varepsilon>0$ arbitrarily and take a $K>0$ satisfying $\sup _{\left|y^{d}\right| \geqslant K, y^{d} \in S_{m}} h\left(y^{d}\right)<\varepsilon$ and $[-K, K] \cap S_{m} \neq \emptyset$. Then

$$
\begin{aligned}
& \sup _{x \in \Omega_{\mu}}\left|p_{t} f(x)-f(x)\right| \\
& \quad \leqslant \sup _{x^{\prime} \in \mathbb{R}^{d-1},\left|x^{d}\right| \geqslant K, x^{d} \in S_{m}}\left|p_{t} f(x)-f(x)\right|+\sup _{x^{\prime} \in \mathbb{R}^{d-1},\left|x^{d}\right| \leqslant K, x^{d} \in S_{m}}\left|p_{t} f(x)-f(x)\right| \\
& \quad \equiv I_{t}+I I_{t} .
\end{aligned}
$$

By means of (4.15), we have a $t_{o}>0$ such that

$$
\left|p_{t}^{D} h\left(x^{d}\right)-h\left(x^{d}\right)\right|<\varepsilon, \quad x^{d} \in S_{m}, 0<t \leqslant t_{o},
$$

from which

$$
p_{t}^{D} h\left(x^{d}\right) \leqslant h\left(x^{d}\right)+\varepsilon<2 \varepsilon, \quad\left|x^{d}\right| \geqslant K, x^{d} \in S_{m}, 0<t \leqslant t_{o} .
$$

Combining this with (4.34),

$$
I_{t}<3 \varepsilon, \quad 0<t \leqslant t_{o} .
$$

Since the diffusion processes $\mathbf{X}^{\prime}$ and $D$ are conservative, we find by Lemma 4.4 that

$$
\begin{aligned}
\left|p_{t} f(x)-f(x)\right| & \leqslant E_{0}^{B}\left[E_{x^{\prime}}^{\prime}\left[\left|f\left(X_{u}^{\prime}, \xi\right)-f\left(x^{\prime}, \xi\right)\right|\right]\right]+E_{0}^{B}\left[\left|f\left(x^{\prime}, \xi\right)-f\left(x^{\prime}, x^{d}\right)\right|\right] \\
& \equiv I I I(t, x)+I V(t, x),
\end{aligned}
$$

where $u=\mathbf{f}\left(\tau\left(t ; x^{d}\right) ; x^{d}\right), \xi=\gamma\left(B_{\phi\left(\tau\left(t ; x^{d}\right) ; x^{d}\right)}+s\left(x^{d}\right)\right)$. Noting (4.31), we find that

$$
\begin{aligned}
I I I(t, x) & =E_{0}^{B}\left[\int_{\mathbb{R}^{d-1}} p^{\prime}\left(u, x^{\prime}, y^{\prime}\right)\left|f\left(y^{\prime}, \xi\right)-f\left(x^{\prime}, \xi\right)\right| \mathrm{d} y^{\prime}\right] \\
& \leqslant C_{3} E_{0}^{B}\left[\int_{\mathbb{R}^{d-1}} \mathrm{e}^{-C_{4}\left|y^{\prime}\right|^{2}}\left|f\left(x^{\prime}+\sqrt{u} y^{\prime}, \xi\right)-f\left(x^{\prime}, \xi\right)\right| \mathrm{d} y^{\prime}\right] .
\end{aligned}
$$

We note that $u \rightarrow 0$ as $t \downarrow 0$ uniformly in $x^{d} \in[-K, K] \cap S_{m}$ by virtue of (4.29) and (4.30). Hence

$$
\limsup _{t \downarrow 0} \sup _{x^{\prime} \in \mathbb{R}^{d-1},\left|x^{\prime}\right| \leqslant K, x^{d} \in S_{m}, \eta \in S_{m}}\left|f\left(x^{\prime}+\sqrt{u} y^{\prime}, \eta\right)-f\left(x^{\prime}, \eta\right)\right|=0, \quad y^{\prime} \in \mathbb{R}^{d-1},
$$

from which

$$
\limsup _{t \downarrow 0} \sup _{x^{\prime} \in \mathbb{R}^{d-1},\left|x^{\prime}\right| \leqslant K, x^{d} \in S_{m}} \operatorname{III}(t, x)
$$




$$
\begin{aligned}
\leqslant & C_{3} E_{0}^{B}\left[\int_{\mathbb{R}^{d-1}} \mathrm{e}^{-C_{4}\left|y^{\prime}\right|^{2}}\right. \\
& \left.\times \limsup _{t \downarrow 0} \sup _{x^{\prime} \in \mathbb{R}^{d-1},\left|x^{\prime}\right| \leqslant K, x^{d} \in S_{m}, \eta \in S_{m}}\left|f\left(x^{\prime}+\sqrt{u} y^{\prime}, \eta\right)-f\left(x^{\prime}, \eta\right)\right| \mathrm{d} y^{\prime}\right] \\
= & 0 .
\end{aligned}
$$

By means of (4.11) and Lemma 4.8,

$$
\begin{aligned}
\sup _{\left|x^{d}\right| \leqslant K, x^{d} \in S_{m}}\left|\xi-x^{d}\right| & =\sup _{\left|x^{d}\right| \leqslant K, x^{d} \in S_{m}}\left|\gamma\left(B_{\phi\left(\tau\left(t ; x^{d}\right) ; x^{d}\right)}+s\left(x^{d}\right)\right)-\gamma\left(s\left(x^{d}\right)\right)\right| \\
& \leqslant \Lambda \sup _{\left|x^{d}\right| \leqslant K, x^{d} \in S_{m}}\left|B_{\phi\left(\tau\left(t ; x^{d}\right) ; x^{d}\right)}\right| \leqslant \Lambda \max _{0 \leqslant s \leqslant \Lambda \tau_{K}(t)}\left|B_{s}\right| \rightarrow 0 \quad \text { as } t \downarrow 0,
\end{aligned}
$$

from which

$$
\begin{aligned}
& \limsup \sup _{t \downarrow 0} \sup _{x^{\prime} \in \mathbb{R}^{d-1},\left|x^{d}\right| \leqslant K, x^{d} \in S_{m}} I V(t, x) \\
& \leqslant E_{0}^{B}\left[\limsup _{t \downarrow 0} \sup _{x^{\prime} \in \mathbb{R}^{d-1},\left|x^{d}\right| \leqslant K, x^{d} \in S_{m}}\left|f\left(x^{\prime}, \xi\right)-f\left(x^{\prime}, x^{d}\right)\right|\right]=0 .
\end{aligned}
$$

(4.37) and (4.38) yield $\lim _{t \downarrow 0} I I_{t}=0$. Combining this with (4.36), we obtain (4.35).

Proof of Theorem 4.3. - Let us fix an $\varepsilon>0$ arbitrarily. By virtue of Proposition 4.10, there is a $K_{1}>0$ such that

$$
\sup _{x^{\prime} \in \mathbb{R}^{d-1},\left|x^{d}\right| \geqslant K_{1}, x^{d} \in S_{m}}\left|p_{t} f(x)\right|<\varepsilon .
$$

Further, by means of Proposition 4.9, there is a $K_{2}>K_{1}$ such that

$$
\sup _{\left|x^{\prime}\right| \geqslant K_{2},\left|x^{d}\right| \leqslant K_{1}, x^{d} \in S_{m}}\left|p_{t} f(x)\right|<\varepsilon .
$$

Consequently

$$
\sup _{|x| \geqslant 2 K_{2}, x \in \Omega_{\mu}}\left|p_{t} f(x)\right|<\varepsilon .
$$

Proposition 4.7 coupled with this implies (4.7). (4.8) is already shown in Proposition 4.11.

\section{Dirichlet form of the limit process}

In this section, we derive the Dirichlet form $(\mathcal{E}, \mathcal{F})$ of the limit process $\mathbf{X}$ defined by (3.8). $\mathbf{X}$ is a time changed process of the conservative diffusion process $\mathbf{Y}$ defined by (4.3), which is the skew product of $\mathbf{X}^{\prime}$ and $\Xi$ with respect to $\mathbf{f}$ defined by (3.4). Since $\mathrm{d} \xi+\rho(\mathrm{d} \xi)$ charges no set of zero $\mathcal{E}^{\Xi}$-capacity, by virtue of [6], the Dirichlet space $\left(\mathcal{E}^{Y}, \mathcal{F}^{Y}\right)$ associated with $\mathbf{Y}$ is regular on $L^{2}\left(\mathbb{R}^{d}, \mathrm{~d} x\right)$ and has $C_{0}^{\infty}\left(\mathbb{R}^{d}\right)$ as a core. Further $\left(\mathcal{E}^{Y}, \mathcal{F}^{Y}\right)$ is given by 


$$
\begin{aligned}
& \mathcal{E}^{Y}(u, u)= \frac{1}{2} \int_{\mathbb{R}^{d}} \sum_{i, j=1}^{d-1} a^{i j}\left(x^{\prime}\right) \partial_{x^{i}} u(x) \partial_{x^{j}} u(x) d x^{\prime}\left(\mathrm{d} x^{d}+\rho\left(\mathrm{d} x^{d}\right)\right) \\
&+\frac{1}{2} \int_{\mathbb{R}^{d}} a^{d d}\left(x^{d}\right) \partial_{x^{d}} u(x) \partial_{x^{d}} u(x) \mathrm{d} x, \\
& \mathcal{F}^{Y}=\left\{u \in L^{2}\left(\mathbb{R}^{d}, \mathrm{~d} x\right): \begin{array}{l}
\partial_{x^{i}} u \in L^{2}\left(\mathbb{R}^{d}, \mathrm{~d} x^{\prime}\left(\mathrm{d} x^{d}+\rho\left(\mathrm{d} x^{d}\right)\right)\right), 1 \leqslant i \leqslant d-1 \\
\partial_{x^{d}} u \in L^{2}\left(\mathbb{R}^{d}, \mathrm{~d} x\right)
\end{array}\right\} .
\end{aligned}
$$

We next note that $\mu(\mathrm{d} x)=\mathrm{d} x^{\prime} m\left(\mathrm{~d} x^{d}\right)$ charges no set of zero $\mathcal{E}^{Y}$-capacity. For this, it is enough to show that, for every compact set $B \subset \mathbb{R}^{d}$, there is a positive constant $C$ such that

$$
\int_{B}|u(x)| \mu(\mathrm{d} x) \leqslant C \sqrt{\mathcal{E}_{1}^{Y}(u, u)}, \quad u \in C_{0}^{\infty}\left(\mathbb{R}^{d}\right),
$$

that is, $I_{B}(x) \mu(\mathrm{d} x)$ is of finite energy integral, where $\mathcal{E}_{1}^{Y}(u, u)=\mathcal{E}^{Y}(u, u)+(u, u)_{L^{2}\left(\mathbb{R}^{d}\right)}$. Let $\Phi$ be an element of $C_{0}^{\infty}(\mathbb{R})$ such that $\Phi(\xi)=1$ for $\xi \in I \equiv\left\{x^{d}:\left(x^{\prime}, x^{d}\right) \in B\right.$ for some $\left.x^{\prime} \in \mathbb{R}^{d-1}\right\}$. Then it is easy to see that

$$
\int_{B}|u| \mathrm{d} \mu \leqslant\left\{\left\|\partial_{x^{d}} u\right\|_{L^{2}\left(\mathbb{R}^{d}\right)}\|\Phi\|_{L^{2}(\mathbb{R})}+\|u\|_{L^{2}\left(\mathbb{R}^{d}\right)}\left\|\Phi^{\prime}\right\|_{L^{2}(\mathbb{R})}\right\} \mu(B)^{1 / 2} m(I)^{1 / 2},
$$

from which (5.1) follows. Further we note that $A(t)$ defined by (3.6) is a positive continuous additive functional of the diffusion process $\mathbf{Y}$ and $P_{x}(A(t)>0, t>$ $0)=1, \quad x \in \Omega_{\mu}$, because of $P_{x^{d}}^{\Xi}(A(t)>0, \quad t>0)=1, \quad x^{d} \in S_{m}$. Employing Theorem 6.2.1 in [7], we then see that the Dirichlet space $(\mathcal{E}, \mathcal{F})$ is regular on $L^{2}\left(\Omega_{\mu}, \mu\right)$ and has $\left.C_{0}^{\infty}\left(\mathbb{R}^{d}\right)\right|_{\Omega_{\mu}}$ as a core.

If $S_{m}=\mathbb{R}$, then the Dirichlet form $(\mathcal{E}, \mathcal{F})$ is given by (2.3) with the domain $\mathcal{F}=$ $\left\{u \in L^{2}\left(\mathbb{R}^{d}, \mu\right): \partial_{x^{i}} u \in L^{2}\left(\mathbb{R}^{d}, \mathrm{~d} x^{\prime}\left(\mathrm{d} x^{d}+\rho\left(\mathrm{d} x^{d}\right)\right)\right), 1 \leqslant i \leqslant d-1, \partial_{x^{d}} u \in L^{2}\left(\mathbb{R}^{d}, \mathrm{~d} x\right)\right\}$, which is immediately derived from [7, Theorem 6.2.1].

We are thus restricted to the case where $\mathbb{R} \backslash S_{m} \neq \emptyset$. In this case, $p_{t} f(x)$ is regarded as a function of $x \in \Omega_{\mu}$ in the sense of Proposition 4.1. Following [7, Theorem 6.2.1], we see that the restricted transition operator $\left\{p_{t}, t>0\right\}$ to $\Omega_{\mu}$ determines a strongly continuous semigroup $\left\{T_{t}, t>0\right\}$ on $L^{2}\left(\Omega_{\mu}, \mu\right)$, which is associated with the Dirichlet form $(\mathcal{E}, \mathcal{F})$ on $L^{2}\left(\Omega_{\mu}, \mu\right)$ being regarded as the Dirichlet form of the time changed process $\mathbf{X}$.

We now assume that

$$
\begin{gathered}
a^{d d}(\xi)=1, \quad \xi \in \mathbb{R} \backslash S_{m}, \\
\operatorname{supp}[\rho] \subset S_{m} .
\end{gathered}
$$

$\mathbb{R} \backslash S_{m}$ is expressed as $\mathbb{R} \backslash S_{m}=\bigcup_{k \in K} I_{k}$, a finite or a countable disjoint union of open intervals $I_{k}=\left(a_{k}, b_{k}\right)$ with the end points belonging to $S_{m} \cup\{-\infty, \infty\}$. We put $\Gamma=\mathbb{R}^{d-1}$ and $\Omega_{k}=\Gamma \times I_{k}$. Let $G_{k}(x, y)$ be the Green function on $\Omega_{k}$ of the Dirichlet boundary value problem corresponding to the operator $\frac{1}{2}\left\{\sum_{i, j=1}^{d-1} \partial_{x^{i}}\left(a^{i j} \partial_{x^{j}}\right)+\partial_{x^{d}} \partial_{x^{d}}\right\}$ 
in the weak sense. Note that $a^{d d}\left(x^{d}\right)=1$ on $\Omega_{k}$ because of (5.2). We introduce two functions $U_{k}$ and $V_{k}$ on $\Gamma \times \Gamma$ accompanied with the interval $I_{k}$, where we denote the right and left partial derivatives in $x^{d}$ by $\partial_{x^{d}}^{+}$and $\partial_{x^{d}}^{-}$respectively.

$$
\begin{aligned}
U_{k}\left(x^{\prime}, y^{\prime}\right) & = \begin{cases}\partial_{x^{d}}^{+} \partial_{y^{d}}^{+} G_{k}\left(\left(x^{\prime}, a_{k}\right),\left(y^{\prime}, a_{k}\right)\right), & \text { if } a_{k}>-\infty, \\
\partial_{x^{d}}^{-} \partial_{y^{d}}^{-} G_{k}\left(\left(x^{\prime}, b_{k}\right),\left(y^{\prime}, b_{k}\right)\right), & \text { if } b_{k}<\infty,\end{cases} \\
V_{k}\left(x^{\prime}, y^{\prime}\right) & =-\partial_{x^{d}}^{+} \partial_{y^{d}}^{-} G_{k}\left(\left(x^{\prime}, a_{k}\right),\left(y^{\prime}, b_{k}\right)\right) \\
& =-\partial_{x^{d}}^{-} \partial_{y^{d}}^{+} G_{k}\left(\left(x^{\prime}, b_{k}\right),\left(y^{\prime}, a_{k}\right)\right), \quad \text { if }-\infty<a_{k}<b_{k}<\infty .
\end{aligned}
$$

We notice that the right hand sides of (5.4) are the same whenever $-\infty<a_{k}<b_{k}<\infty$ (see (5.13) with (5.16) below).

THEOREM 5.1. - Assume that $S_{m} \neq \mathbb{R}$ and (5.2), (5.3) hold. Then the Dirichlet space $(\mathcal{E}, \mathcal{F})$ of $\mathbf{X}$ is regular on $L^{2}\left(\Omega_{\mu}, \mu\right)$ and has $\left.C_{0}^{\infty}\left(\mathbb{R}^{d}\right)\right|_{\Omega_{\mu}}$ as a core. For $\left.u \in C_{0}^{\infty}\left(\mathbb{R}^{d}\right)\right|_{\Omega_{\mu}}$, the Dirichlet form $\mathcal{E}(u, u)$ is given by

$$
\begin{aligned}
\mathcal{E}(u, u)= & \frac{1}{2} \int_{\Omega_{\mu}}\left\{\sum_{i, j=1}^{d-1} a^{i j}\left(x^{\prime}\right) \partial_{x^{i}} u(x) \partial_{x^{j}} u(x)+a^{d d}\left(x^{d}\right) \partial_{x^{d}}^{*} u(x) \partial_{x^{d}}^{*} u(x)\right\} \mathrm{d} x \\
& +\frac{1}{2} \int_{\Omega_{\mu}} \sum_{i, j=1}^{d-1} a^{i j}\left(x^{\prime}\right) \partial_{x^{i}} u(x) \partial_{x^{j}} u(x) \mathrm{d} x^{\prime} \rho\left(\mathrm{d} x^{d}\right) \\
& +\frac{1}{8} \sum_{k \in K:} \sum_{-\infty<a_{k}<b_{k} \leqslant \infty} \int_{\Gamma \times \Gamma}\left\{u\left(x^{\prime}, a_{k}\right)-u\left(y^{\prime}, a_{k}\right)\right\}^{2} U_{k}\left(x^{\prime}, y^{\prime}\right) \mathrm{d} x^{\prime} \mathrm{d} y^{\prime} \\
& +\frac{1}{8} \sum_{k \in K:} \sum_{-\infty \leqslant a_{k}<b_{k}<\infty} \int_{\Gamma \times \Gamma}\left\{u\left(x^{\prime}, b_{k}\right)-u\left(y^{\prime}, b_{k}\right)\right\}^{2} U_{k}\left(x^{\prime}, y^{\prime}\right) \mathrm{d} x^{\prime} \mathrm{d} y^{\prime} \\
& +\frac{1}{4} \sum_{k \in K:} \sum_{-\infty<a_{k}<b_{k}<\infty} \int_{\Gamma \times \Gamma}\left\{u\left(x^{\prime}, a_{k}\right)-u\left(y^{\prime}, b_{k}\right)\right\}^{2} V_{k}\left(x^{\prime}, y^{\prime}\right) \mathrm{d} x^{\prime} \mathrm{d} y^{\prime},
\end{aligned}
$$

where the first term of the right hand side vanishes in case where $\left|S_{m}\right|=0 .{ }^{4}$ In case where $\left|S_{m}\right|>0$, $\partial_{x^{d}}^{*} u$ is defined by

$$
\partial_{x^{d}}^{*} u\left(x^{\prime}, x^{d}\right)=\lim _{\xi \rightarrow x^{d}, \xi \in S_{m}}\left\{u\left(x^{\prime}, \xi\right)-u\left(x^{\prime}, x^{d}\right)\right\} /\left(\xi-x^{d}\right),
$$

which exists for every $x^{\prime} \in \Gamma$ and $\mathrm{d} x^{d}$-a.e. $x^{d} \in S_{m}$.

In order to prove Theorem 5.1, we prepare some lemmas. We first note that $G_{k}(x, y)$ is expressed as

$$
G_{k}(x, y)=\int_{0}^{\infty} p^{\prime}\left(t, x^{\prime}, y^{\prime}\right) q_{k}\left(t, x^{d}, y^{d}\right) \mathrm{d} t, \quad x, y \in \Omega_{k},
$$

\footnotetext{
${ }^{4}|E|$ stands for the Lebesgue measure of a set $E$.
} 
where $p^{\prime}\left(t, x^{\prime}, y^{\prime}\right)$ is the transition probability density of $\mathbf{X}^{\prime}$ and $q_{k}(t, \xi, \eta)$ is that of the absorbing Brownian motion on $I_{k}$, which is given as follows. If $-\infty=a_{k}<b_{k}<\infty$,

$$
q_{k}(t, \xi, \eta)=\frac{1}{\sqrt{2 \pi t}}\left\{\mathrm{e}^{-|\xi-\eta|^{2} / 2 t}-\mathrm{e}^{-\left|\xi+\eta-2 b_{k}\right|^{2} / 2 t}\right\} .
$$

If $-\infty<a_{k}<b_{k}=\infty$,

$$
q_{k}(t, \xi, \eta)=\frac{1}{\sqrt{2 \pi t}}\left\{\mathrm{e}^{-|\xi-\eta|^{2} / 2 t}-\mathrm{e}^{-\left|\xi+\eta-2 a_{k}\right|^{2} / 2 t}\right\} .
$$

In the case where $-\infty<a_{k}<b_{k}<\infty$, we have the following two expressions:

$$
\begin{aligned}
q_{k}(t, \xi, \eta) & =\frac{2}{b_{k}-a_{k}} \sum_{n=1}^{\infty} \mathrm{e}^{-\left(n \pi /\left(b_{k}-a_{k}\right)\right)^{2} t / 2} \sin \left(\frac{\xi-a_{k}}{b_{k}-a_{k}} n \pi\right) \sin \left(\frac{\eta-a_{k}}{b_{k}-a_{k}} n \pi\right) \\
& =\frac{1}{\sqrt{2 \pi t}} \sum_{n=-\infty}^{\infty}\left\{\mathrm{e}^{-\left|\eta-\xi+2 n\left(b_{k}-a_{k}\right)\right|^{2} / 2 t}-\mathrm{e}^{-\left|\eta+\xi-2 a_{k}+2 n\left(b_{k}-a_{k}\right)\right|^{2} / 2 t}\right\} .
\end{aligned}
$$

Accordingly,

$$
U_{k}\left(x^{\prime}, y^{\prime}\right)=\int_{0}^{\infty} p^{\prime}\left(t, x^{\prime}, y^{\prime}\right) \alpha_{k}(t) \mathrm{d} t
$$

where

$$
\begin{aligned}
\alpha_{k}(t)= & \partial_{\xi}^{-} \partial_{\eta}^{-} q_{k}\left(t, b_{k}, b_{k}\right)=\sqrt{\frac{2}{\pi t^{3}}}, \quad \text { if }-\infty=a_{k}<b_{k}<\infty, \\
\alpha_{k}(t)= & \partial_{\xi}^{+} \partial_{\eta}^{+} q_{k}\left(t, a_{k}, a_{k}\right)=\sqrt{\frac{2}{\pi t^{3}}}, \quad \text { if }-\infty<a_{k}<b_{k}=\infty, \\
\alpha_{k}(t)= & \partial_{\xi}^{+} \partial_{\eta}^{+} q_{k}\left(t, a_{k}, a_{k}\right)=\partial_{\xi}^{-} \partial_{\eta}^{-} q_{k}\left(t, b_{k}, b_{k}\right) \\
= & \frac{2}{b_{k}-a_{k}} \sum_{n=1}^{\infty}\left(\frac{n \pi}{b_{k}-a_{k}}\right)^{2} \mathrm{e}^{-\left(n \pi /\left(b_{k}-a_{k}\right)\right)^{2} t / 2}, \\
& \text { if }-\infty<a_{k}<b_{k}<\infty .
\end{aligned}
$$

Further, if $-\infty<a_{k}<b_{k}<\infty$,

$$
V_{k}\left(x^{\prime}, y^{\prime}\right)=\int_{0}^{\infty} p^{\prime}\left(t, x^{\prime}, y^{\prime}\right) \beta_{k}(t) \mathrm{d} t,
$$

where

$$
\begin{aligned}
\beta_{k}(t) & =-\partial_{\xi}^{+} \partial_{\eta}^{-} q_{k}\left(t, a_{k}, b_{k}\right)=-\partial_{\xi}^{-} \partial_{\eta}^{+} q_{k}\left(t, b_{k}, a_{k}\right) \\
& =\frac{2}{b_{k}-a_{k}} \sum_{n=1}^{\infty}(-1)^{n-1}\left(\frac{n \pi}{b_{k}-a_{k}}\right)^{2} \mathrm{e}^{-\left(n \pi /\left(b_{k}-a_{k}\right)\right)^{2} t / 2}
\end{aligned}
$$




$$
=\sqrt{\frac{2}{\pi t^{3}}} \sum_{n=-\infty}^{\infty}\left\{\frac{(2 n+1)^{2}\left(b_{k}-a_{k}\right)^{2}}{t}-1\right\} \mathrm{e}^{-(2 n+1)^{2}\left(b_{k}-a_{k}\right)^{2} / 2 t} .
$$

We note the following estimates.

LEMMA 5.2. - (i) Let $-\infty=a_{k}<b_{k}<\infty$ or $-\infty<a_{k}<b_{k}=\infty$. Then there exist positive constants $C_{5}$ and $C_{6}$ independent of $I_{k}$ such that for every $x^{\prime}, y^{\prime} \in \Gamma$,

$$
C_{5}\left|x^{\prime}-y^{\prime}\right|^{-d} \leqslant U_{k}\left(x^{\prime}, y^{\prime}\right) \leqslant C_{6}\left|x^{\prime}-y^{\prime}\right|^{-d} .
$$

(ii) Let $-\infty<a_{k}<b_{k}<\infty$. Then there exist positive constants $T_{o}$ and $C_{i}(i=$ $7,8, \ldots, 12)$ independent of $I_{k}$ such that, if $\left|x^{\prime}-y^{\prime}\right| \leqslant T_{o}\left(b_{k}-a_{k}\right)$, then

$$
\begin{gathered}
C_{7}\left|x^{\prime}-y^{\prime}\right|^{-d} \leqslant U_{k}\left(x^{\prime}, y^{\prime}\right) \leqslant C_{8}\left|x^{\prime}-y^{\prime}\right|^{-d}, \\
C_{9}\left(b_{k}-a_{k}\right)^{-d} \leqslant V_{k}\left(x^{\prime}, y^{\prime}\right) \leqslant C_{10}\left(b_{k}-a_{k}\right)^{-d},
\end{gathered}
$$

and if $\left|x^{\prime}-y^{\prime}\right| \geqslant T_{o}\left(b_{k}-a_{k}\right)$, then for $W=U_{k}, V_{k}$,

$$
\begin{aligned}
& C_{11}\left(b_{k}-a_{k}\right)^{-d / 2-1}\left|x^{\prime}-y^{\prime}\right|^{-d / 2+1} \mathrm{e}^{-\sqrt{2 C_{2}} \pi\left|x^{\prime}-y^{\prime}\right| /\left(b_{k}-a_{k}\right)} \\
& \quad \leqslant W\left(x^{\prime}, y^{\prime}\right) \leqslant C_{12}\left(b_{k}-a_{k}\right)^{-d / 2-1}\left|x^{\prime}-y^{\prime}\right|^{-d / 2+1} \mathrm{e}^{-\sqrt{2 C_{4}} \pi\left|x^{\prime}-y^{\prime}\right| /\left(b_{k}-a_{k}\right)},
\end{aligned}
$$

where $C_{2}$ and $C_{4}$ are positive constants which appeared in (4.31).

Proof. - (i) Let $-\infty=a_{k}<b_{k}<\infty$ or $-\infty<a_{k}<b_{k}=\infty$. Then we immediately obtain (5.20) from (4.31) and (5.13)-(5.15).

(ii) Let $-\infty<a_{k}<b_{k}<\infty$. We divide the proof into four steps. For simplicity we set $\theta=b_{k}-a_{k}$. In the following, $c_{i}(i=1,2, \ldots)$ stand for positive constants independent of $t, x^{\prime}, y^{\prime}, \theta$ and $k$.

(Step 1) First we remark that

$$
\begin{aligned}
c_{1} t^{-3 / 2} \leqslant \alpha_{k}(t) \leqslant c_{2} t^{-3 / 2} & \text { if } 0<t \leqslant \theta^{2} / 2, \\
c_{3} \theta^{-3} \mathrm{e}^{-\pi^{2} t / 2 \theta^{2}} \leqslant \alpha_{k}(t) \leqslant c_{4} \theta^{-3} \mathrm{e}^{-\pi^{2} t / 2 \theta^{2}} & \text { if } t \geqslant \theta^{2} / 2 .
\end{aligned}
$$

It follows from (5.16) that

$$
2 \pi^{2} \theta^{-3} \mathrm{e}^{-\pi^{2} t / 2 \theta^{2}} \leqslant \alpha_{k}(t) \leqslant 2 \pi^{2} \theta^{-3} \mathrm{e}^{-\pi^{2} t / 2 \theta^{2}}\left\{1+\sum_{n=2}^{\infty} n^{2} \mathrm{e}^{-n^{2} \pi^{2} t / 4 \theta^{2}}\right\}, \quad t>0 .
$$

If $t \geqslant \theta^{2} / 2$, then

$$
\sum_{n=2}^{\infty} n^{2} \mathrm{e}^{-n^{2} \pi^{2} t / 4 \theta^{2}} \leqslant \sum_{n=2}^{\infty} n^{2} \mathrm{e}^{-n^{2}} \leqslant \int_{0}^{\infty} \xi^{2} \mathrm{e}^{-\xi^{2}} \mathrm{~d} \xi=\frac{\sqrt{\pi}}{4}
$$

Combining this with (5.26), we get (5.25).

Since

$$
\begin{aligned}
& (1 / 4)(n+1)^{2} \mathrm{e}^{-(n+1)^{2} \pi^{2} t / 2 \theta^{2}} \leqslant \xi^{2} \mathrm{e}^{-\xi^{2} \pi^{2} t / 2 \theta^{2}} \leqslant 4 n^{2} \mathrm{e}^{-n^{2} \pi^{2} t / 2 \theta^{2}}, \\
& n \leqslant \xi \leqslant n+1, n \in \mathbb{N}
\end{aligned}
$$


we see that

$$
\begin{aligned}
\sum_{n=1}^{\infty} n^{2} \mathrm{e}^{-n^{2} \pi^{2} t / 2 \theta^{2}} & \leqslant \mathrm{e}^{-\pi^{2} t / 2 \theta^{2}}+4 \int_{1}^{\infty} \xi^{2} \mathrm{e}^{-\xi^{2} \pi^{2} t / 2 \theta^{2}} \mathrm{~d} \xi \\
& \leqslant 1+\frac{8 \sqrt{2} \theta^{3}}{\pi^{3} t^{3 / 2}} \int_{\pi \sqrt{t} / \sqrt{2} \theta}^{\infty} \xi^{2} \mathrm{e}^{-\xi^{2}} \mathrm{~d} \xi \\
& \leqslant \theta^{3} t^{-3 / 2}\left\{\theta^{-3} t^{3 / 2}+2 \sqrt{2} \pi^{-5 / 2}\right\} \\
\sum_{n=1}^{\infty} n^{2} \mathrm{e}^{-n^{2} \pi^{2} t / 2 \theta^{2}} & \geqslant \frac{1}{4} \int_{1}^{\infty} \xi^{2} \mathrm{e}^{-\xi^{2} \pi^{2} t / 2 \theta^{2}} \mathrm{~d} \xi \\
& =\frac{\theta^{3}}{\sqrt{2} \pi^{3} t^{3 / 2}} \int_{\pi \sqrt{t} / \sqrt{2} \theta}^{\infty} \xi^{2} \mathrm{e}^{-\xi^{2} \mathrm{~d} \xi}
\end{aligned}
$$

Therefore, if $t \leqslant \theta^{2} / 2$,

$$
\frac{\theta^{3}}{\sqrt{2} \pi^{3} t^{3 / 2}} \int_{\pi / 2}^{\infty} \xi^{2} \mathrm{e}^{-\xi^{2}} \mathrm{~d} \xi \leqslant \sum_{n=1}^{\infty} n^{2} \mathrm{e}^{-n^{2} \pi^{2} t / 2 \theta^{2}} \leqslant\left(2^{-3 / 2}+2 \sqrt{2} \pi^{-5 / 2}\right) \theta^{3} t^{-3 / 2}
$$

from which we get (5.24).

(Step 2) We next note that

$$
\begin{array}{cl}
c_{5} \theta^{2} t^{-5 / 2} \mathrm{e}^{-\theta^{2} / 2 t} \leqslant \beta_{k}(t) \leqslant c_{6} \theta^{2} t^{-5 / 2} \mathrm{e}^{-\theta^{2} / 2 t} & \text { if } 0<t \leqslant \theta^{2} / 2, \\
c_{7} \theta^{-3} \mathrm{e}^{-\pi^{2} t / 2 \theta^{2}} \leqslant \beta_{k}(t) \leqslant c_{8} \theta^{-3} \mathrm{e}^{-\pi^{2} t / 2 \theta^{2}} & \text { if } t \geqslant \theta^{2} / 2 .
\end{array}
$$

By means of (5.18),

$$
\begin{aligned}
\beta_{k}(t)= & 2 \pi^{2} \theta^{-3}\left[\mathrm{e}^{-\pi^{2} t / 2 \theta^{2}}\left\{1-4 \mathrm{e}^{-(3 / 2) \pi^{2} t / \theta^{2}}\right\}\right. \\
& \left.+\sum_{n=1}^{\infty}\left\{(2 n+1)^{2} \mathrm{e}^{-(2 n+1)^{2} \pi^{2} t / 2 \theta^{2}}-(2 n+2)^{2} \mathrm{e}^{-(2 n+2)^{2} \pi^{2} t / 2 \theta^{2}}\right\}\right] \\
\leqslant & 2 \pi^{2} \theta^{-3} \mathrm{e}^{-\pi^{2} t / 2 \theta^{2}}\left[1+\sum_{n=1}^{\infty}(2 n+1)^{2} \mathrm{e}^{-\left\{(2 n+1)^{2}-1\right\} \pi^{2} t / 2 \theta^{2}}\right] \\
\leqslant & 2 \pi^{2} \theta^{-3} \mathrm{e}^{-\pi^{2} t / 2 \theta^{2}}\left[1+4 \sum_{n=2}^{\infty} n^{2} \mathrm{e}^{-n^{2} \pi^{2} t / 2 \theta^{2}}\right] .
\end{aligned}
$$

If $t \geqslant \theta^{2} / 2$, then

$$
\sum_{n=2}^{\infty} n^{2} \mathrm{e}^{-n^{2} \pi^{2} t / 2 \theta^{2}} \leqslant \sum_{n=2}^{\infty} n^{2} \mathrm{e}^{-n^{2}}<\sqrt{\pi} / 4
$$


from which

$$
\beta_{k}(t)<2 \pi^{2}(1+\sqrt{\pi}) \theta^{-3} \mathrm{e}^{-\pi^{2} t / 2 \theta^{2}} .
$$

We also see that, if $t \geqslant \theta^{2} / 2$, then $f(\xi):=\xi \mathrm{e}^{-\xi \pi^{2} t / 2 \theta^{2}}$ is decreasing in $\xi \in[1, \infty)$, and hence $\sum_{n=1}^{\infty}\{f(2 n+1)-f(2 n+2)\}>0$, from which

$$
\beta_{k}(t) \geqslant 2 \pi^{2} \theta^{-3} \mathrm{e}^{-\pi^{2} t / 2 \theta^{2}}\left\{1-4 \mathrm{e}^{-3 \pi^{2} / 4}\right\}
$$

This coupled with (5.29) implies (5.28).

Let $0<t \leqslant \theta^{2} / 2$. Then

$$
\frac{(2 n+1)^{2} \theta^{2}}{t}-1 \geqslant \frac{\theta^{2}}{t}-1 \geqslant \frac{\theta^{2}}{2 t}, \quad n=0, \pm 1, \pm 2, \ldots
$$

Therefore, by means of (5.19),

$$
\beta_{k}(t) \geqslant \frac{1}{\sqrt{2 \pi}} \theta^{2} t^{-5 / 2} \mathrm{e}^{-\theta^{2} / 2 t}
$$

On the other hand,

$$
\beta_{k}(t) \leqslant 2 \sqrt{2 / \pi} \theta^{2} t^{-5 / 2} \mathrm{e}^{-\theta^{2} / 2 t}\left\{1+\sum_{n=1}^{\infty}(2 n+1)^{2} \mathrm{e}^{-\left\{(2 n+1)^{2}-1\right\} \theta^{2} / 2 t}\right\}
$$

Since

$$
\sum_{n=1}^{\infty}(2 n+1)^{2} \mathrm{e}^{-\left\{(2 n+1)^{2}-1\right\} \theta^{2} / 2 t} \leqslant 4 \sum_{n=2}^{\infty} n^{2} \mathrm{e}^{-n^{2}}<\sqrt{\pi},
$$

we have

$$
\beta_{k}(t) \leqslant 2 \sqrt{2 / \pi}(1+\sqrt{\pi}) \theta^{2} t^{-5 / 2} \mathrm{e}^{-\theta^{2} / 2 t} .
$$

Combining this with (5.30), we get (5.27).

(Step 3) Let $C$ be a positive number and put

$$
\begin{aligned}
& J_{1}=\int_{0}^{\theta^{2} / 2} t^{-d / 2-1} \mathrm{e}^{-C\left|x^{\prime}-y^{\prime}\right|^{2} / t} \mathrm{~d} t, \\
& J_{2}=\theta^{2} \int_{0}^{\theta^{2} / 2} t^{-d / 2-2} \mathrm{e}^{-C\left|x^{\prime}-y^{\prime}\right|^{2} / t} \mathrm{e}^{-\theta^{2} / 2 t} \mathrm{~d} t, \\
& J_{3}=\theta^{-3} \int_{\theta^{2} / 2}^{\infty} t^{-(d-1) / 2} \mathrm{e}^{-C\left|x^{\prime}-y^{\prime}\right|^{2} / t} \mathrm{e}^{-\pi^{2} t / 2 \theta^{2}} \mathrm{~d} t .
\end{aligned}
$$

Then there is a positive constant $T_{o}$ independent of $\theta$ such that, if $\left|x^{\prime}-y^{\prime}\right| \leqslant T_{o} \theta$, then 


$$
\begin{aligned}
c_{9}\left|x^{\prime}-y^{\prime}\right|^{-d} & \leqslant J_{1} \leqslant c_{10}\left|x^{\prime}-y^{\prime}\right|^{-d}, \\
c_{11} \theta^{-d} & \leqslant J_{2} \leqslant c_{12} \theta^{-d}, \\
0 & <J_{3} \leqslant c_{13} \theta^{-d},
\end{aligned}
$$

and if $\left|x^{\prime}-y^{\prime}\right| \geqslant T_{o} \theta$, then

$$
\begin{aligned}
& 0<J_{1} \leqslant c_{14} \theta^{-d / 2-1}\left|x^{\prime}-y^{\prime}\right|^{-d / 2+1} \mathrm{e}^{-\sqrt{2 C} \pi\left|x^{\prime}-y^{\prime}\right| / \theta}, \\
& 0<J_{2} \leqslant c_{15} \theta^{-d / 2-1}\left|x^{\prime}-y^{\prime}\right|^{-d / 2+1} \mathrm{e}^{-\sqrt{2 C} \pi\left|x^{\prime}-y^{\prime}\right| / \theta}, \\
& c_{16} \theta^{-d / 2-1}\left|x^{\prime}-y^{\prime}\right|^{-d / 2+1} \mathrm{e}^{-\sqrt{2 C} \pi\left|x^{\prime}-y^{\prime}\right| / \theta} \\
& \leqslant J_{3} \leqslant c_{17} \theta^{-d / 2-1}\left|x^{\prime}-y^{\prime}\right|^{-d / 2+1} \mathrm{e}^{-\sqrt{2 C} \pi\left|x^{\prime}-y^{\prime}\right| / \theta} .
\end{aligned}
$$

Here is a proof. It is known that

$$
\int_{0}^{\infty} t^{-v-1} \mathrm{e}^{-a^{2} / 4 t} \mathrm{e}^{-p t} \mathrm{~d} t=2^{v+1} p^{v / 2} a^{-v} K_{v}(a \sqrt{p}),
$$

where $a>0, p>0$, and $K_{v}(\xi)$ is the modified Bessel function. Therefore

$$
\begin{aligned}
J_{4} & \equiv \int_{0}^{\infty} t^{-(d-1) / 2} \mathrm{e}^{-C\left|x^{\prime}-y^{\prime}\right|^{2} / t} \mathrm{e}^{-\pi^{2} t / 2 \theta^{2}} \mathrm{~d} t \\
& =c_{18} \theta^{-(d-3) / 2}\left|x^{\prime}-y^{\prime}\right|^{-(d-3) / 2} K_{(d-3) / 2}\left(\sqrt{2 C} \pi\left|x^{\prime}-y^{\prime}\right| / \theta\right) .
\end{aligned}
$$

Since $\lim _{\xi \rightarrow \infty} \frac{K_{\mathrm{v}}(\xi)}{\mathrm{e}^{-\xi} \sqrt{\pi / 2 \xi}}=1$, there is a $T_{1}>0$ such that

$$
\frac{1}{2} \sqrt{\frac{\pi}{2 \xi}} \mathrm{e}^{-\xi} \leqslant K_{v}(\xi) \leqslant 2 \sqrt{\frac{\pi}{2 \xi}} \mathrm{e}^{-\xi}, \quad \xi \geqslant T_{1} .
$$

Accordingly, if $\left|x^{\prime}-y^{\prime}\right| / \theta \geqslant T_{1} / \sqrt{2 C} \pi$, then

$$
\begin{aligned}
& c_{19} \theta^{-d / 2+2}\left|x^{\prime}-y^{\prime}\right|^{-d / 2+1} \mathrm{e}^{-\sqrt{2 C} \pi\left|x^{\prime}-y^{\prime}\right| / \theta} \\
& \quad \leqslant J_{4} \leqslant c_{20} \theta^{-d / 2+2}\left|x^{\prime}-y^{\prime}\right|^{-d / 2+1} \mathrm{e}^{-\sqrt{2 C} \pi\left|x^{\prime}-y^{\prime}\right| / \theta} .
\end{aligned}
$$

Put

$$
J_{5}=\int_{0}^{\theta^{2} / 2} t^{-(d-1) / 2} \mathrm{e}^{-C\left|x^{\prime}-y^{\prime}\right|^{2} / t} \mathrm{e}^{-\pi^{2} t / 2 \theta^{2}} \mathrm{~d} t
$$

Then

$$
\begin{aligned}
J_{5} & \leqslant \mathrm{e}^{-C\left|x^{\prime}-y^{\prime}\right|^{2} / \theta^{2}} \int_{0}^{\theta^{2} / 2} t^{-(d-1) / 2} \mathrm{e}^{-C\left|x^{\prime}-y^{\prime}\right|^{2} / 2 t} \mathrm{~d} t \\
& =2^{(d-3) / 2}\left|x^{\prime}-y^{\prime}\right|^{-d+3} \mathrm{e}^{-C\left|x^{\prime}-y^{\prime}\right|^{2} / \theta^{2}} \int_{0}^{\theta^{2} /\left|x^{\prime}-y^{\prime}\right|^{2}} t^{-(d-1) / 2} \mathrm{e}^{-C / t} \mathrm{~d} t .
\end{aligned}
$$


We take a $T_{2} \geqslant \min \left\{T_{1} / \sqrt{2 C} \pi, 2\right\}$ such that for every $\xi \geqslant T_{2}$,

$$
\xi^{2} \mathrm{e}^{-C \xi^{2}} \int_{0}^{1 / \xi^{2}} t^{-d / 2-2} \mathrm{e}^{-C / t} \mathrm{~d} t \leqslant c_{19} \mathrm{e}^{-\sqrt{2 C} \pi \xi} .
$$

Hence, if $\left|x^{\prime}-y^{\prime}\right| / \theta \geqslant T_{2}(\geqslant 2)$, then by means of (5.38),

$$
\begin{aligned}
J_{5} & \leqslant 2^{(d-3) / 2} c_{19} \theta^{2}\left|x^{\prime}-y^{\prime}\right|^{-d+1} \mathrm{e}^{-\sqrt{2 C} \pi\left|x^{\prime}-y^{\prime}\right| / \theta} \\
& =2^{(d-3) / 2} c_{19}\left(\theta /\left|x^{\prime}-y^{\prime}\right|\right)^{d / 2} \theta^{-d / 2+2}\left|x^{\prime}-y^{\prime}\right|^{-d / 2+1} \mathrm{e}^{-\sqrt{2 C} \pi\left|x^{\prime}-y^{\prime}\right| / \theta} \\
& <2^{-3 / 2} c_{19} \theta^{-d / 2+2}\left|x^{\prime}-y^{\prime}\right|^{-d / 2+1} \mathrm{e}^{-\sqrt{2 C} \pi\left|x^{\prime}-y^{\prime}\right| / \theta} .
\end{aligned}
$$

Combining this with (5.37), we find that if $\left|x^{\prime}-y^{\prime}\right| / \theta \geqslant T_{2}$, then

$$
J_{3}=\theta^{-3}\left(J_{4}-J_{5}\right) \geqslant 2^{-1} c_{19} \theta^{-d / 2-1}\left|x^{\prime}-y^{\prime}\right|^{-d / 2+1} \mathrm{e}^{-\sqrt{2 C} \pi\left|x^{\prime}-y^{\prime}\right| / \theta} .
$$

Noting that $J_{3} \leqslant \theta^{-3} J_{4}$ and putting $T_{o}=T_{2}$, we get (5.36).

We also notice that

$$
J_{3} \leqslant \theta^{-3} \int_{\theta^{2} / 2}^{\infty} t^{-(d-1) / 2} \mathrm{e}^{-\pi^{2} t / 2 \theta^{2}} \mathrm{~d} t=\theta^{-d} \int_{1 / 2}^{\infty} t^{-(d-1) / 2} \mathrm{e}^{-\pi^{2} t / 2} \mathrm{~d} t,
$$

which shows (5.33).

We next notice that

$$
J_{1}=2^{d / 2}\left|x^{\prime}-y^{\prime}\right|^{-d} \int_{0}^{\theta^{2} /\left|x^{\prime}-y^{\prime}\right|^{2}} t^{-d / 2-1} \mathrm{e}^{-2 C / t} \mathrm{~d} t
$$

and hence, if $\left|x^{\prime}-y^{\prime}\right| / \theta \leqslant T_{o}$, then

$$
2^{d / 2}\left|x^{\prime}-y^{\prime}\right|^{-d} \int_{0}^{1 / T_{o}^{2}} t^{-d / 2-1} \mathrm{e}^{-2 C / t} \mathrm{~d} t \leqslant J_{1} \leqslant 2^{d / 2}\left|x^{\prime}-y^{\prime}\right|^{-d} \int_{0}^{\infty} t^{-d / 2-1} \mathrm{e}^{-2 C / t} \mathrm{~d} t .
$$

We thus obtain (5.31).

If $\left|x^{\prime}-y^{\prime}\right| / \theta \geqslant T_{o}$, by means of (5.39),

$$
\begin{aligned}
J_{1} & \leqslant 2^{d / 2}\left|x^{\prime}-y^{\prime}\right|^{-d} \mathrm{e}^{-C\left|x^{\prime}-y^{\prime}\right| / \theta^{2}} \int_{0}^{\theta^{2} /\left|x^{\prime}-y^{\prime}\right|^{2}} t^{-d / 2-1} \mathrm{e}^{-C / t} \mathrm{~d} t \\
& \leqslant 2^{d / 2} c_{19}\left(\left|x^{\prime}-y^{\prime}\right| / \theta\right)^{-2}\left|x^{\prime}-y^{\prime}\right|^{-d} \mathrm{e}^{-\sqrt{2 C} \pi\left|x^{\prime}-y^{\prime}\right| / \theta} \\
& =2^{d / 2} c_{19}\left(\left|x^{\prime}-y^{\prime}\right| / \theta\right)^{-d / 2-3} \theta^{-d / 2-1}\left|x^{\prime}-y^{\prime}\right|^{-d / 2+1} \mathrm{e}^{-\sqrt{2 C} \pi\left|x^{\prime}-y^{\prime}\right| / \theta} \\
& \leqslant 2^{d / 2} c_{19} T_{o}^{-d / 2-3} \theta^{-d / 2-1}\left|x^{\prime}-y^{\prime}\right|^{-d / 2+1} \mathrm{e}^{-\sqrt{2 C} \pi\left|x^{\prime}-y^{\prime}\right| / \theta},
\end{aligned}
$$

which shows (5.34). 
Since

$$
J_{2}=\theta^{-d} \int_{0}^{1 / 2} t^{-d / 2-2} \mathrm{e}^{-C\left|x^{\prime}-y^{\prime}\right|^{2} / \theta^{2} t} \mathrm{e}^{-1 / 2 t} \mathrm{~d} t
$$

we have always

$$
J_{2} \leqslant \theta^{-d} \int_{0}^{1 / 2} t^{-d / 2-2} \mathrm{e}^{-1 / 2 t} \mathrm{~d} t .
$$

On the other hand, if $\left|x^{\prime}-y^{\prime}\right| / \theta \leqslant T_{o}$, then

$$
J_{2} \geqslant \theta^{-d} \int_{0}^{1 / 2} t^{-d / 2-2} \mathrm{e}^{-C T_{o}^{2} / t} \mathrm{e}^{-1 / 2 t} \mathrm{~d} t .
$$

This coupled with (5.40) implies (5.32).

If $\left|x^{\prime}-y^{\prime}\right| / \theta \geqslant T_{o}$, then by means of (5.39),

$$
\begin{aligned}
J_{2} & \leqslant \theta^{2} \mathrm{e}^{-C\left|x^{\prime}-y^{\prime}\right|^{2} / \theta^{2}} \int_{0}^{\theta^{2} / 2} t^{-d / 2-2} \mathrm{e}^{-C\left|x^{\prime}-y^{\prime}\right|^{2} / 2 t} \mathrm{~d} t \\
& =2^{d / 2+1} \theta^{2}\left|x^{\prime}-y^{\prime}\right|^{-d-2} \mathrm{e}^{-C\left|x^{\prime}-y^{\prime}\right|^{2} / \theta^{2}} \int_{0}^{\theta^{2} /\left|x^{\prime}-y^{\prime}\right|^{2}} t^{-d / 2-2} \mathrm{e}^{-C / t} \mathrm{~d} t \\
& \leqslant 2^{d / 2+1} c_{19} \theta^{-d / 2-1}\left|x^{\prime}-y^{\prime}\right|^{-d / 2+1}\left(\left|x^{\prime}-y^{\prime}\right| / \theta\right)^{-d / 2-5} \mathrm{e}^{-\sqrt{2 C} \pi\left|x^{\prime}-y^{\prime}\right| / \theta} \\
& \leqslant 2^{d / 2+1} c_{19} T_{o}^{-d / 2-5} \theta^{-d / 2-1}\left|x^{\prime}-y^{\prime}\right|^{-d / 2+1} \mathrm{e}^{-\sqrt{2 C} \pi\left|x^{\prime}-y^{\prime}\right| / \theta} .
\end{aligned}
$$

This shows (5.35).

(Step 4) We give a proof of (5.21), (5.22) and (5.23). By virtue of (4.31), (5.13), (5.24) and (5.25),

$$
\begin{aligned}
& c_{20}\left\{\int_{0}^{\theta^{2} / 2} t^{-d / 2-1} \mathrm{e}^{-C_{2}\left|x^{\prime}-y^{\prime}\right|^{2} / t} \mathrm{~d} t+\theta^{-3} \int_{\theta^{2} / 2}^{\infty} t^{-(d-1) / 2} \mathrm{e}^{-C_{2}\left|x^{\prime}-y^{\prime}\right|^{2} / t} \mathrm{e}^{-\pi^{2} t / 2 \theta^{2}} \mathrm{~d} t\right\} \\
& \leqslant U_{k}\left(x^{\prime}, y^{\prime}\right) \\
& \leqslant c_{21}\left\{\int_{0}^{\theta^{2} / 2} t^{-d / 2-1} \mathrm{e}^{-C_{4}\left|x^{\prime}-y^{\prime}\right|^{2} / t} \mathrm{~d} t+\theta^{-3} \int_{\theta^{2} / 2}^{\infty} t^{-(d-1) / 2} \mathrm{e}^{-C_{4}\left|x^{\prime}-y^{\prime}\right|^{2} / t} \mathrm{e}^{-\pi^{2} t / 2 \theta^{2}} \mathrm{~d} t\right\} .
\end{aligned}
$$

If $\left|x^{\prime}-y^{\prime}\right| \leqslant T_{o} \theta$, then by means of (5.31) and (5.33),

$$
c_{22}\left|x^{\prime}-y^{\prime}\right|^{-d} \leqslant U_{k}\left(x^{\prime}, y^{\prime}\right) \leqslant c_{23}\left|x^{\prime}-y^{\prime}\right|^{-d},
$$

which shows (5.21). If $\left|x^{\prime}-y^{\prime}\right| \geqslant T_{o} \theta$, then by means of (5.34) and (5.36), 


$$
\begin{aligned}
& c_{24} \theta^{-d / 2-1}\left|x^{\prime}-y^{\prime}\right|^{-d / 2+1} \mathrm{e}^{-\sqrt{2 C_{2}} \pi\left|x^{\prime}-y^{\prime}\right| / \theta} \\
& \leqslant U_{k}\left(x^{\prime}, y^{\prime}\right) \leqslant c_{25} \theta^{-d / 2-1}\left|x^{\prime}-y^{\prime}\right|^{-d / 2+1} \mathrm{e}^{-\sqrt{2 C_{4}} \pi\left|x^{\prime}-y^{\prime}\right| / \theta} .
\end{aligned}
$$

This shows (5.23) with $W=U_{k}$.

By virtue of (4.31), (5.17), (5.27) and (5.28),

$$
\begin{aligned}
c_{26}\left\{\theta^{2} \int_{0}^{\theta^{2} / 2} t^{-d / 2-2} \mathrm{e}^{-C_{2}\left|x^{\prime}-y^{\prime}\right|^{2} / t} \mathrm{e}^{-\theta^{2} / 2 t} \mathrm{~d} t\right. \\
\left.+\theta^{-3} \int_{\theta^{2} / 2}^{\infty} t^{-(d-1) / 2} \mathrm{e}^{-C_{2}\left|x^{\prime}-y^{\prime}\right|^{2} / t} \mathrm{e}^{-\pi^{2} t / 2 \theta^{2}} \mathrm{~d} t\right\} \\
\leqslant V_{k}\left(x^{\prime}, y^{\prime}\right) \\
\leqslant c_{27}\left\{\theta^{2} \int_{0}^{\theta^{2} / 2} t^{-d / 2-2} \mathrm{e}^{-C_{4}\left|x^{\prime}-y^{\prime}\right|^{2} / t} \mathrm{e}^{-\theta^{2} / 2 t} \mathrm{~d} t\right. \\
\left.+\theta^{-3} \int_{\theta^{2} / 2}^{\infty} t^{-(d-1) / 2} \mathrm{e}^{-C_{4}\left|x^{\prime}-y^{\prime}\right|^{2} / t} \mathrm{e}^{-\pi^{2} t / 2 \theta^{2}} \mathrm{~d} t\right\} .
\end{aligned}
$$

If $\left|x^{\prime}-y^{\prime}\right| \leqslant T_{o} \theta$, then by means of (5.32) and (5.33),

$$
c_{28} \theta^{-d} \leqslant V_{k}\left(x^{\prime}, y^{\prime}\right) \leqslant c_{29} \theta^{-d},
$$

which is (5.22). If $\left|x^{\prime}-y^{\prime}\right| \geqslant T_{o} \theta$, then by means of (5.35) and (5.36),

$$
\begin{aligned}
& c_{30} \theta^{-d / 2-1}\left|x^{\prime}-y^{\prime}\right|^{-d / 2+1} \mathrm{e}^{-\sqrt{2 C_{2}} \pi\left|x^{\prime}-y^{\prime}\right| / \theta} \\
& \leqslant V_{k}\left(x^{\prime}, y^{\prime}\right) \leqslant c_{31} \theta^{-d / 2-1}\left|x^{\prime}-y^{\prime}\right|^{-d / 2+1} \mathrm{e}^{-\sqrt{2 C_{2}} \pi\left|x^{\prime}-y^{\prime}\right| / \theta} .
\end{aligned}
$$

We thus get (5.23) with $W=V_{k}$.

Since $\left.C_{0}^{\infty}\left(\mathbb{R}^{d}\right)\right|_{\Omega_{\mu}}$ is a core of $(\mathcal{E}, \mathcal{F})$, we fix a $u \in C_{0}^{\infty}\left(\mathbb{R}^{d}\right)$ and set $\varphi=\left.u\right|_{\Omega_{\mu}}$. Then $\varphi \in \mathcal{F}$ and

$$
\mathcal{E}(\varphi, \varphi)=\mathcal{E}^{Y}\left(H_{\Omega_{\mu}} u, H_{\Omega_{\mu}} u\right),
$$

where $H_{E} u(x)=E_{x}\left[u\left(Y_{\sigma_{E}^{Y}}\right): \sigma_{E}^{Y}<\infty\right], x \in \mathbb{R}^{d}, \sigma_{E}^{Y}$ is the first hitting time of $\mathbf{Y}$ for a set $E: \sigma_{E}^{Y}=\inf \left\{t>0: Y_{t} \in E\right\}$. Note that $H_{\Omega_{\mu}} u \in \mathcal{F}^{Y}$. We put

$$
\begin{aligned}
\mathcal{E}_{\Omega_{\mu}}(v, v)= & \frac{1}{2} \int_{\Omega_{\mu}} \sum_{i, j=1}^{d-1} a^{i j}\left(x^{\prime}\right) \partial_{x^{i}} v(x) \partial_{x^{j}} v(x) d x^{\prime}\left(\mathrm{d} x^{d}+\rho\left(\mathrm{d} x^{d}\right)\right) \\
& +\frac{1}{2} \int_{\Omega_{\mu}} a^{d d}\left(x^{d}\right) \partial_{x^{d}} v(x) \partial_{x^{d}} v(x) \mathrm{d} x, \\
\mathcal{E}_{\Omega_{k}}(v, v)= & \frac{1}{2} \int_{\Omega_{k}} \sum_{i, j=1}^{d-1} a^{i j}\left(x^{\prime}\right) \partial_{x^{i}} v(x) \partial_{x^{j}} v(x) \mathrm{d} x+\frac{1}{2} \int_{\Omega_{k}} \partial_{x^{d}} v(x) \partial_{x^{d}} v(x) \mathrm{d} x .
\end{aligned}
$$


Noting (5.2) and (5.3), we see that

$$
\mathcal{E}(\varphi, \varphi)=\mathcal{E}_{\Omega_{\mu}}\left(H_{\Omega_{\mu}} u, H_{\Omega_{\mu}} u\right)+\sum_{k \in K} \mathcal{E}_{\Omega_{k}}\left(H_{\Omega_{\mu}} u, H_{\Omega_{\mu}} u\right) .
$$

LEMMA 5.3. - It holds that

$$
\begin{aligned}
& \mathcal{E}_{\Omega_{\mu}}\left(H_{\Omega_{\mu}} u, H_{\Omega_{\mu}} u\right) \\
& =\frac{1}{2} \int_{\Omega_{\mu}}\left\{\sum_{i, j=1}^{d-1} a^{i j}\left(x^{\prime}\right) \partial_{x^{i}} \varphi(x) \partial_{x^{j}} \varphi(x)+a^{d d}\left(x^{d}\right) \partial_{x^{d}}^{*} \varphi(x) \partial_{x^{d}}^{*} \varphi(x)\right\} \mathrm{d} x \\
& \quad+\frac{1}{2} \int_{\Omega_{\mu}} \sum_{i, j=1}^{d-1} a^{i j}\left(x^{\prime}\right) \partial_{x^{i}} \varphi(x) \partial_{x^{j}} \varphi(x) \mathrm{d} x^{\prime} \rho\left(\mathrm{d} x^{d}\right),
\end{aligned}
$$

where $\partial_{x^{d}}^{*}$ is defined by (5.7), and the first term of the right hand side vanishes in case where $\left|S_{m}\right|=0$.

Proof. - It is obvious that $H_{\Omega_{\mu}} u(x)=u(x)=\varphi(x), x \in \Omega_{\mu}$, because of $P_{x}\left(\sigma_{\Omega_{\mu}}^{Y}=\right.$ $0)=1$ for $x \in \Omega_{\mu}$. Since $H_{\Omega_{\mu}} u \in \mathcal{F}^{Y}$,

$$
\begin{aligned}
\partial_{x^{i}} H_{\Omega_{\mu}} u(x) & =\frac{\partial}{\partial x^{i}} u(x)=\frac{\partial}{\partial x^{i}} \varphi(x), \quad \mathrm{d} x^{\prime}\left(\mathrm{d} x^{d}+\rho\left(\mathrm{d} x^{d}\right)\right) \text {-a.e. } x \in \Omega_{\mu}, \\
1 \leqslant i \leqslant d & -1, \\
\partial_{x^{d}} H_{\Omega_{\mu}} u(x) & =\frac{\partial}{\partial x^{d}} u(x), \quad \mathrm{d} x^{\prime} \mathrm{d} x^{d} \text {-a.e. } x \in \Omega_{\mu} .
\end{aligned}
$$

Assume that $\left|S_{m}\right|>0$. Then

$$
\lim _{\varepsilon \downarrow 0}\left|\left(x^{d}, x^{d}+\varepsilon\right] \cap S_{m}\right| / \varepsilon=\lim _{\varepsilon \downarrow 0}\left|\left(x^{d}-\varepsilon, x^{d}\right] \cap S_{m}\right| / \varepsilon=1, \quad \text { d } x^{d} \text {-a.e. } x^{d} \in S_{m},
$$

from which

$$
\begin{aligned}
\frac{\partial}{\partial x^{d}} u\left(x^{\prime}, x^{d}\right) & =\lim _{\xi \rightarrow x^{d}, \xi \in S_{m}}\left\{u\left(x^{\prime}, \xi\right)-u\left(x^{\prime}, x^{d}\right)\right\} /\left(\xi-x^{d}\right) \\
& =\lim _{\xi \rightarrow x^{d}, \xi \in S_{m}}\left\{\varphi\left(x^{\prime}, \xi\right)-\varphi\left(x^{\prime}, x^{d}\right)\right\} /\left(\xi-x^{d}\right)=\partial_{x^{d}}^{*} \varphi\left(x^{\prime}, x^{d}\right),
\end{aligned}
$$

for every $x^{\prime} \in \Gamma$ and $\mathrm{d} x^{d}$-a.e. $x^{d} \in S_{m}$. We thus get the conclusion of the lemma.

We are going to derive an explicit form of $\mathcal{E}_{\Omega_{k}}\left(H_{\Omega_{\mu}} u, H_{\Omega_{\mu}} u\right)$.

LemmA 5.4. - (i) Let $-\infty=a_{k}<b_{k}<\infty$. Then

$$
\mathcal{E}_{\Omega_{k}}\left(H_{\Omega_{\mu}} u, H_{\Omega_{\mu}} u\right)=\frac{1}{8} \iint_{\Gamma \times \Gamma}\left\{\varphi\left(x^{\prime}, b_{k}\right)-\varphi\left(y^{\prime}, b_{k}\right)\right\}^{2} U_{k}\left(x^{\prime}, y^{\prime}\right) \mathrm{d} x^{\prime} \mathrm{d} y^{\prime} .
$$

(ii) Let $-\infty<a_{k}<b_{k}=\infty$. Then

$$
\mathcal{E}_{\Omega_{k}}\left(H_{\Omega_{\mu}} u, H_{\Omega_{\mu}} u\right)=\frac{1}{8} \iint_{\Gamma \times \Gamma}\left\{\varphi\left(x^{\prime}, a_{k}\right)-\varphi\left(y^{\prime}, a_{k}\right)\right\}^{2} U_{k}\left(x^{\prime}, y^{\prime}\right) \mathrm{d} x^{\prime} \mathrm{d} y^{\prime} .
$$


Proof. - We assume $b_{k}<\infty$ and write $b$ in place of $b_{k}$. The assumption (5.3) implies that $P_{\xi}^{B}\left(\mathbf{f}(t)=t, 0 \leqslant t<\sigma_{S_{m}}^{B}\right)=1$ for $\xi \in I_{k}$. We also note that $P_{x}\left(\sigma_{\Omega_{\mu}}^{Y}=\sigma_{S_{m}}^{B}=\sigma_{b}^{B}<\right.$ $\infty)=1$ for $x \in \Omega_{k}$. Therefore

$$
\begin{aligned}
& H_{\Omega_{\mu}} u(x)=E_{x}\left[\varphi\left(X^{\prime}\left(\sigma_{b}^{B}\right), b\right)\right]=-\frac{1}{2} \int_{\Gamma} \varphi\left(y^{\prime}, b\right) \partial_{y^{d}}^{-} G_{k}\left(x,\left(y^{\prime}, b\right)\right) \mathrm{d} y^{\prime}, \\
& P_{x}\left(X^{\prime}\left(\sigma_{b}^{B}\right) \in \mathrm{d} y^{\prime}\right)=-\frac{1}{2} \partial_{y^{d}}^{-} G_{k}\left(x,\left(y^{\prime}, b\right)\right) \mathrm{d} y^{\prime},
\end{aligned}
$$

for $x \in \Omega_{k}, y^{\prime} \in \Gamma$. By means of Green's formula and (5.43),

$$
\begin{aligned}
\mathcal{E}_{\Omega_{k}}\left(H_{\Omega_{\mu}} u, H_{\Omega_{\mu}} u\right)= & \frac{1}{2} \int_{\Gamma} H_{\Omega_{\mu}} u\left(x^{\prime}, b\right) \partial_{x^{d}}^{-} H_{\Omega_{\mu}} u\left(x^{\prime}, b\right) \mathrm{d} x^{\prime} \\
= & \left.\frac{1}{2} \int_{\Gamma} \varphi\left(x^{\prime}, b\right) \partial_{x^{d}}^{-} E_{x}\left[\varphi\left(X^{\prime}\left(\sigma_{b}^{B}\right), b\right)\right]\right|_{x^{d}=b} \mathrm{~d} x^{\prime} \\
= & -\left.\frac{1}{4} \int_{\Gamma} \partial_{x^{d}}^{-} E_{x}\left[\left\{\varphi\left(x^{\prime}, b\right)-\varphi\left(X^{\prime}\left(\sigma_{b}^{B}\right), b\right)\right\}^{2}\right]\right|_{x^{d}=b} \mathrm{~d} x^{\prime} \\
& +\left.\frac{1}{4} \int_{\Gamma} \partial_{x^{d}}^{-} E_{x}\left[\varphi\left(x^{\prime}, b\right)^{2}+\varphi\left(X^{\prime}\left(\sigma_{b}^{B}\right), b\right)^{2}\right]\right|_{x^{d}=b} \mathrm{~d} x^{\prime} \\
\equiv & J_{1}+J_{2} .
\end{aligned}
$$

By virtue of (5.44), (5.4) and (5.20),

$$
\begin{aligned}
J_{1} & =\frac{1}{8} \iint_{\Gamma \times \Gamma}\left\{\varphi\left(x^{\prime}, b\right)-\varphi\left(y^{\prime}, b\right)\right\}^{2} \partial_{x^{d}}^{-} \partial_{y^{d}}^{-} G_{k}\left(\left(x^{\prime}, b\right),\left(y^{\prime}, b\right)\right) \mathrm{d} x^{\prime} \mathrm{d} y^{\prime} \\
& =\frac{1}{8} \iint_{\Gamma \times \Gamma}\left\{\varphi\left(x^{\prime}, b\right)-\varphi\left(y^{\prime}, b\right)\right\}^{2} U_{k}\left(x^{\prime}, y^{\prime}\right) \mathrm{d} x^{\prime} \mathrm{d} y^{\prime}<\infty .
\end{aligned}
$$

Since $H_{\Omega_{\mu}} 1(x)=P_{x^{d}}^{B}\left(\sigma_{b}^{B}<\infty\right)=1$ and hence $\partial_{x^{d}} H_{\Omega_{\mu}} 1(x)=0$, by using Green's formula again,

$$
J_{2}=\left.\frac{1}{4} \int_{\Gamma} \partial_{x^{d}}^{-} E_{x}\left[\varphi\left(X^{\prime}\left(\sigma_{b}^{B}\right), b\right)^{2}\right]\right|_{x^{d}=b} \mathrm{~d} x^{\prime}=\frac{1}{2} \mathcal{E}_{\Omega_{k}}\left(H_{\Omega_{\mu}} 1, H_{\Omega_{\mu}}\left(u^{2}\right)\right)=0 .
$$

Thus we arrive at the first assertion. The second assertion is obtained in the same way as above.

LEMMA 5.5. - Let $-\infty<a_{k}<b_{k}<\infty$. Then

$$
\begin{aligned}
\mathcal{E}_{\Omega_{k}}\left(H_{\Omega_{\mu}} u, H_{\Omega_{\mu}} u\right)= & \frac{1}{8} \iint_{\Gamma \times \Gamma}\left\{\varphi\left(x^{\prime}, a_{k}\right)-\varphi\left(y^{\prime}, a_{k}\right)\right\}^{2} U_{k}\left(x^{\prime}, y^{\prime}\right) \mathrm{d} x^{\prime} \mathrm{d} y^{\prime} \\
& +\frac{1}{8} \iint_{\Gamma \times \Gamma}\left\{\varphi\left(x^{\prime}, b_{k}\right)-\varphi\left(y^{\prime}, b_{k}\right)\right\}^{2} U_{k}\left(x^{\prime}, y^{\prime}\right) \mathrm{d} x^{\prime} \mathrm{d} y^{\prime}
\end{aligned}
$$




$$
+\frac{1}{4} \iint_{\Gamma \times \Gamma}\left\{\varphi\left(x^{\prime}, a_{k}\right)-\varphi\left(y^{\prime}, b_{k}\right)\right\}^{2} V_{k}\left(x^{\prime}, y^{\prime}\right) \mathrm{d} x^{\prime} \mathrm{d} y^{\prime} .
$$

Proof. - We set $a=a_{k}, \quad b=b_{k}$. Since $P_{x}\left(\sigma_{\Omega_{\mu}}^{Y}=\sigma_{S_{m}}^{B}=\sigma_{a}^{B} \wedge \sigma_{b}^{B}<\infty\right)=1$ for $x \in \Omega_{k}$, where $r \wedge s=\min \{r, s\}$, we have

$$
\begin{gathered}
H_{\Omega_{\mu}} u(x)=E_{x}\left[\varphi\left(X^{\prime}\left(\sigma_{a}^{B} \wedge \sigma_{b}^{B}\right), B\left(\sigma_{a}^{B} \wedge \sigma_{b}^{B}\right)\right)\right] \\
=\frac{1}{2} \int_{\Gamma}\left\{\varphi\left(y^{\prime}, a\right) \partial_{y^{d}}^{+} G_{k}\left(x,\left(y^{\prime}, a\right)\right)-\varphi\left(y^{\prime}, b\right) \partial_{y^{d}}^{-} G_{k}\left(x,\left(y^{\prime}, b\right)\right)\right\} \mathrm{d} y^{\prime}, \\
P_{x}\left(X^{\prime}\left(\sigma_{a}^{B}\right) \in \mathrm{d} y^{\prime}, \sigma_{a}^{B}<\sigma_{b}^{B}\right)=\frac{1}{2} \partial_{y^{d}}^{+} G_{k}\left(x,\left(y^{\prime}, a\right)\right) \mathrm{d} y^{\prime}, \\
P_{x}\left(X^{\prime}\left(\sigma_{b}^{B}\right) \in \mathrm{d} y^{\prime}, \sigma_{b}^{B}<\sigma_{a}^{B}\right)=-\frac{1}{2} \partial_{y^{d}}^{-} G_{k}\left(x,\left(y^{\prime}, b\right)\right) \mathrm{d} y^{\prime},
\end{gathered}
$$

for $x \in \Omega_{k}$ and $y \in \Gamma$. By means of Green's formula and (5.45), we get

$$
\begin{aligned}
& \mathcal{E}_{\Omega_{k}}\left(H_{\Omega_{\mu}} u, H_{\Omega_{\mu}} u\right) \\
& =\frac{1}{2} \int_{\Gamma}\left\{H_{\Omega_{\mu}} u\left(x^{\prime}, b\right) \partial_{x^{d}}^{-} H_{\Omega_{\mu}} u\left(x^{\prime}, b\right)-H_{\Omega_{\mu}} u\left(x^{\prime}, a\right) \partial_{x^{d}}^{+} H_{\Omega_{\mu}} u\left(x^{\prime}, a\right)\right\} \mathrm{d} x^{\prime} \\
& =\frac{1}{2} \int_{\Gamma}\left\{\left.\varphi\left(x^{\prime}, b\right) \partial_{x^{d}}^{-} E_{x}\left[\varphi\left(X^{\prime}\left(\sigma_{a}^{B} \wedge \sigma_{b}^{B}\right), B\left(\sigma_{a}^{B} \wedge \sigma_{b}^{B}\right)\right)\right]\right|_{x^{d}=b}\right. \\
& \left.-\left.\varphi\left(x^{\prime}, a\right) \partial_{x^{d}}^{+} E_{x}\left[\varphi\left(X^{\prime}\left(\sigma_{a}^{B} \wedge \sigma_{b}^{B}\right), B\left(\sigma_{a}^{B} \wedge \sigma_{b}^{B}\right)\right)\right]\right|_{x^{d}=a}\right\} \mathrm{d} x^{\prime} \\
& =\frac{1}{2} \int_{\Gamma}\left\{\left.\varphi\left(x^{\prime}, b\right) \partial_{x^{d}}^{-} E_{x}\left[\varphi\left(X^{\prime}\left(\sigma_{a}^{B}\right), a\right) ; \sigma_{a}^{B}<\sigma_{b}^{B}\right]\right|_{x^{d}=b}\right. \\
& +\left.\varphi\left(x^{\prime}, b\right) \partial_{x^{d}}^{-} E_{x}\left[\varphi\left(X^{\prime}\left(\sigma_{b}^{B}\right), b\right) ; \sigma_{b}^{B}<\sigma_{a}^{B}\right]\right|_{x^{d}=b} \\
& -\left.\varphi\left(x^{\prime}, a\right) \partial_{x^{d}}^{+} E_{x}\left[\varphi\left(X^{\prime}\left(\sigma_{a}^{B}\right), a\right) ; \sigma_{a}^{B}<\sigma_{b}^{B}\right]\right|_{x^{d}=a} \\
& \left.-\left.\varphi\left(x^{\prime}, a\right) \partial_{x^{d}}^{+} E_{x}\left[\varphi\left(X^{\prime}\left(\sigma_{b}^{B}\right), b\right) ; \sigma_{b}^{B}<\sigma_{a}^{B}\right]\right|_{x^{d}=a}\right\} \mathrm{d} x^{\prime} \\
& =-\left.\frac{1}{4} \int_{\Gamma} \partial_{x^{d}}^{-} E_{x}\left[\left\{\varphi\left(x^{\prime}, b\right)-\varphi\left(X^{\prime}\left(\sigma_{a}^{B}\right), a\right)\right\}^{2} ; \sigma_{a}^{B}<\sigma_{b}^{B}\right]\right|_{x^{d}=b} \mathrm{~d} x^{\prime} \\
& +\left.\frac{1}{4} \int_{\Gamma} \partial_{x^{d}}^{-} E_{x}\left[\varphi\left(x^{\prime}, b\right)^{2}+\varphi\left(X^{\prime}\left(\sigma_{a}^{B}\right), a\right)^{2} ; \sigma_{a}^{B}<\sigma_{b}^{B}\right]\right|_{x^{d}=b} \mathrm{~d} x^{\prime} \\
& -\left.\frac{1}{4} \int_{\Gamma} \partial_{x^{d}}^{-} E_{x}\left[\left\{\varphi\left(x^{\prime}, b\right)-\varphi\left(X^{\prime}\left(\sigma_{b}^{B}\right), b\right)\right\}^{2} ; \sigma_{b}^{B}<\sigma_{a}^{B}\right]\right|_{x^{d}=b} \mathrm{~d} x^{\prime} \\
& +\left.\frac{1}{4} \int_{\Gamma} \partial_{x^{d}}^{-} E_{x}\left[\varphi\left(x^{\prime}, b\right)^{2}+\varphi\left(X^{\prime}\left(\sigma_{b}^{B}\right), b\right)^{2} ; \sigma_{b}^{B}<\sigma_{a}^{B}\right]\right|_{x^{d}=b} \mathrm{~d} x^{\prime} \\
& +\left.\frac{1}{4} \int_{\Gamma} \partial_{x^{d}}^{+} E_{x}\left[\left\{\varphi\left(x^{\prime}, a\right)-\varphi\left(X^{\prime}\left(\sigma_{a}^{B}\right), a\right)\right\}^{2} ; \sigma_{a}^{B}<\sigma_{b}^{B}\right]\right|_{x^{d}=a} \mathrm{~d} x^{\prime}
\end{aligned}
$$




$$
\begin{aligned}
& -\left.\frac{1}{4} \int_{\Gamma} \partial_{x^{d}}^{+} E_{x}\left[\varphi\left(x^{\prime}, a\right)^{2}+\varphi\left(X^{\prime}\left(\sigma_{a}^{B}\right), a\right)^{2} ; \sigma_{a}^{B}<\sigma_{b}^{B}\right]\right|_{x^{d}=a} \mathrm{~d} x^{\prime} \\
& +\left.\frac{1}{4} \int_{\Gamma} \partial_{x^{d}}^{+} E_{x}\left[\left\{\varphi\left(x^{\prime}, a\right)-\varphi\left(X^{\prime}\left(\sigma_{b}^{B}\right), b\right)\right\}^{2} ; \sigma_{b}^{B}<\sigma_{a}^{B}\right]\right|_{x^{d}=a} \mathrm{~d} x^{\prime} \\
& -\left.\frac{1}{4} \int_{\Gamma} \partial_{x^{d}}^{+} E_{x}\left[\varphi\left(x^{\prime}, a\right)^{2}+\varphi\left(X^{\prime}\left(\sigma_{b}^{B}\right), b\right)^{2} ; \sigma_{b}^{B}<\sigma_{a}^{B}\right]\right|_{x^{d}=a} \mathrm{~d} x^{\prime} \\
& \equiv J_{1}+J_{2}+J_{3}+J_{4}+J_{5}+J_{6}+J_{7}+J_{8} .
\end{aligned}
$$

By virtue of (5.46), (5.47), (5.4), (5.5), (5.21), (5.22) and (5.23),

$$
\begin{aligned}
J_{1} & =-\frac{1}{8} \int_{\Gamma \times \Gamma}\left\{\varphi\left(x^{\prime}, b\right)-\varphi\left(y^{\prime}, a\right)\right\}^{2} \partial_{x^{d}}^{-} \partial_{y^{d}}^{+} G_{k}\left(\left(x^{\prime}, b\right),\left(y^{\prime}, a\right)\right) \mathrm{d} x^{\prime} \mathrm{d} y^{\prime} \\
& =\frac{1}{8} \iint_{\Gamma \times \Gamma}\left\{\varphi\left(x^{\prime}, b\right)-\varphi\left(y^{\prime}, a\right)\right\}^{2} V_{k}\left(x^{\prime}, y^{\prime}\right) \mathrm{d} x^{\prime} \mathrm{d} y^{\prime}<\infty, \\
J_{3} & =\frac{1}{8} \iint_{\Gamma \times \Gamma}\left\{\varphi\left(x^{\prime}, b\right)-\varphi\left(y^{\prime}, b\right)\right\}^{2} \partial_{x^{d}}^{-} \partial_{y^{d}}^{-} G_{k}\left(\left(x^{\prime}, b\right),\left(y^{\prime}, b\right)\right) \mathrm{d} x^{\prime} \mathrm{d} y^{\prime} \\
& =\frac{1}{8} \iint_{\Gamma \times \Gamma}\left\{\varphi\left(x^{\prime}, b\right)-\varphi\left(y^{\prime}, b\right)\right\}^{2} U_{k}\left(x^{\prime}, y^{\prime}\right) \mathrm{d} x^{\prime} \mathrm{d} y^{\prime}<\infty, \\
J_{5} & =\frac{1}{8} \int_{\Gamma \times \Gamma}\left\{\varphi\left(x^{\prime}, a\right)-\varphi\left(y^{\prime}, a\right)\right\}^{2} U_{k}\left(x^{\prime}, y^{\prime}\right) \mathrm{d} x^{\prime} \mathrm{d} y^{\prime}<\infty, \\
J_{7} & =\frac{1}{8} \int_{\Gamma \times \Gamma}\left\{\varphi\left(x^{\prime}, a\right)-\varphi\left(y^{\prime}, b\right)\right\}^{2} V_{k}\left(x^{\prime}, y^{\prime}\right) \mathrm{d} x^{\prime} \mathrm{d} y^{\prime}=J_{1}<\infty .
\end{aligned}
$$

On the other hand, by means of (5.45) and Green's formula,

$$
\begin{aligned}
J_{2}+ & J_{4}+J_{6}+J_{8} \\
= & \frac{1}{4} \int_{\Gamma}\left\{\left.\partial_{x^{d}}^{-} E_{x}\left[\varphi\left(x^{\prime}, b\right)^{2}+\varphi\left(X^{\prime}\left(\sigma_{a}^{B} \wedge \sigma_{b}^{B}\right), B\left(\sigma_{a}^{B} \wedge \sigma_{b}^{B}\right)\right)^{2}\right]\right|_{x^{d}=b}\right. \\
& \left.-\left.\partial_{x^{d}}^{+} E_{x}\left[\varphi\left(x^{\prime}, a\right)^{2}+\varphi\left(X^{\prime}\left(\sigma_{a}^{B} \wedge \sigma_{b}^{B}\right), B\left(\sigma_{a}^{B} \wedge \sigma_{b}^{B}\right)\right)^{2}\right]\right|_{x^{d}=a}\right\} \mathrm{d} x^{\prime} \\
= & \frac{1}{4} \int_{\Gamma}\left\{\left.\partial_{x^{d}}^{-} E_{x}\left[\varphi\left(X^{\prime}\left(\sigma_{a}^{B} \wedge \sigma_{b}^{B}\right), B\left(\sigma_{a}^{B} \wedge \sigma_{b}^{B}\right)\right)^{2}\right]\right|_{x^{d}=b}\right. \\
& \left.-\left.\partial_{x^{d}}^{+} E_{x}\left[\varphi\left(X^{\prime}\left(\sigma_{a}^{B} \wedge \sigma_{b}^{B}\right), B\left(\sigma_{a}^{B} \wedge \sigma_{b}^{B}\right)\right)^{2}\right]\right|_{x^{d}=a}\right\} \mathrm{d} x^{\prime} \\
= & \frac{1}{2} \mathcal{E}_{\Omega_{k}}\left(H_{\Omega_{\mu}} 1, H_{\Omega_{\mu}}\left(u^{2}\right)\right)=0 .
\end{aligned}
$$

We thus get the conclusion of the lemma. 
Proof of Theorem 5.1. - In view of [7, Theorem 6.2.1], $(\mathcal{E}, \mathcal{F})$ is regular on $L^{2}\left(\Omega_{\mu}, \mu\right)$ and $\left.C_{0}^{\infty}\left(\mathbb{R}^{d}\right)\right|_{\Omega_{\mu}}$ is a core of $(\mathcal{E}, \mathcal{F})$. We then see that, for $\left.u \in C_{0}^{\infty}\left(\mathbb{R}^{d}\right)\right|_{\Omega_{\mu}}, \mathcal{E}(u, u)$ is given by (5.6) because of (5.42) and Lemmas 5.3, 5.4, 5.5.

Example 5.6. - Let $\mathbf{X}^{\prime}$ be the $(d-1)$-dimensional Brownian motion and $\Xi$ the onedimensional one, that is, $a^{i j}\left(x^{\prime}\right)=\delta^{i j}\left(x^{\prime}\right), 1 \leqslant i, j \leqslant d-1$, and $a^{d d}(\xi)=1$. We exhibit Dirichlet spaces corresponding to some $m$ and $\rho$.

(i) Let $m(\mathrm{~d} \xi)=I_{(a, \infty)}(\xi) \mathrm{d} \xi$ and $\rho(\mathrm{d} \xi)=\kappa_{a} \delta_{a}(\mathrm{~d} \xi)$, where $a \in \mathbb{R}, \kappa_{a}$ is a nonnegative constant. Then the corresponding Dirichlet space $(\mathcal{E}, \mathcal{F})$ in Theorem 5.1 is given by

$$
\begin{aligned}
& \mathcal{E}(u, u)=\frac{1}{2} \int_{E_{a}} \sum_{i=1}^{d}\left(\partial_{x^{i}} u\right)^{2} \mathrm{~d} x+\frac{\kappa_{a}}{2} \int_{\Gamma}^{d-1} \sum_{i=1}^{d-1}\left(\partial_{x^{i}} \gamma_{a}^{+} u\right)^{2} \mathrm{~d} x^{\prime} \\
& +\frac{1}{8} \iint_{\Gamma \times \Gamma}\left\{\gamma_{a}^{+} u\left(x^{\prime}\right)-\gamma_{a}^{+} u\left(y^{\prime}\right)\right\}^{2} U_{0}\left(x^{\prime}, y^{\prime}\right) \mathrm{d} x^{\prime} \mathrm{d} y^{\prime}, \\
& \mathcal{F}=\left\{u \in L^{2}\left(E_{a}, \mathrm{~d} x\right): \mathcal{E}(u, u)<\infty\right\} \\
& = \begin{cases}\left\{u \in H^{1}\left(E_{a}\right): \gamma_{a}^{+} u \in H^{1}(\Gamma)\right\}, & \text { if } \kappa_{a}>0, \\
\left\{u \in H^{1}\left(E_{a}\right): \gamma_{a}^{+} u \in H^{1 / 2}(\Gamma)\right\}, & \text { if } \kappa_{a}=0,\end{cases}
\end{aligned}
$$

where $E_{a}=\Gamma \times(a, \infty), \Gamma_{a}=\Gamma \times\{a\}, \gamma_{a}^{+}$is the trace operator on $\Gamma_{a}$ from the domain $E_{a}$, and

$$
\begin{aligned}
U_{0}\left(x^{\prime}, y^{\prime}\right) & =\frac{2}{\sqrt{2 \pi}} \int_{0}^{\infty}(2 \pi t)^{-(d-1) / 2} \mathrm{e}^{-\left|x^{\prime}-y^{\prime}\right|^{2} / 2 t} t^{-3 / 2} \mathrm{~d} t \\
& =2 \pi^{-d / 2} \Gamma(d / 2)\left|x^{\prime}-y^{\prime}\right|^{-d} .
\end{aligned}
$$

$H^{1 / 2}(\Gamma)$ is the fractional order space defined by $H^{1 / 2}(\Gamma)=\left\{u \in L^{2}(\Gamma):\|u\|_{H^{1 / 2}(\Gamma)}\right.$ $<\infty$ \} with the norm

$$
\|u\|_{H^{1 / 2}(\Gamma)}=\|u\|_{L^{2}(\Gamma)}+\left\{\iint_{\Gamma \times \Gamma}\left(u\left(x^{\prime}\right)-u\left(y^{\prime}\right)\right)^{2}\left|x^{\prime}-y^{\prime}\right|^{-d} \mathrm{~d} x^{\prime} \mathrm{d} y^{\prime}\right\}^{1 / 2} .
$$

We always identify $\Gamma_{a}$ with $\Gamma$. In order to see (5.48), it is enough to note that

$$
\begin{gathered}
\left\|\gamma_{a}^{+} u\right\|_{L^{2}(\Gamma)} \leqslant c_{1}\|u\|_{H^{1}\left(E_{a}\right)}, \quad u \in H^{1}\left(E_{a}\right), \\
\|v\|_{H^{1 / 2}(\Gamma)} \leqslant c_{2}\|v\|_{H^{1}(\Gamma)}, \quad v \in H^{1}(\Gamma),
\end{gathered}
$$

for some positive constants $c_{1}$ and $c_{2}$ (cf. [1, Ch. VII]).

(ii) Let $m(\mathrm{~d} \xi)=I_{(a, b)}(\xi) \mathrm{d} \xi$ and $\rho(\mathrm{d} \xi)=\kappa_{a} \delta_{a}(\mathrm{~d} \xi)+\kappa_{b} \delta_{b}(\mathrm{~d} \xi)$, where $-\infty<a<b<$ $\infty$ and $\kappa_{a}, \kappa_{b}$ are nonnegative constants. Then the corresponding Dirichlet space $(\mathcal{E}, \mathcal{F})$ in Theorem 5.1 is given by 


$$
\begin{aligned}
& \mathcal{E}(u, u)= \frac{1}{2} \int_{E_{a b}} \sum_{i=1}^{d}\left(\partial_{x^{i}} u\right)^{2} \mathrm{~d} x \\
&+\frac{\kappa_{a}}{2} \int_{\Gamma} \sum_{i=1}^{d-1}\left(\partial_{x^{i}} \gamma_{a}^{+} u\right)^{2} \mathrm{~d} x^{\prime}+\frac{\kappa_{b}}{2} \int_{\Gamma} \sum_{i=1}^{d-1}\left(\partial_{x^{i}} \gamma_{b}^{-} u\right)^{2} \mathrm{~d} x^{\prime} \\
&+\frac{1}{8} \int_{\Gamma \times \Gamma}\left\{\gamma_{a}^{+} u\left(x^{\prime}\right)-\gamma_{a}^{+} u\left(y^{\prime}\right)\right\}^{2} U_{0}\left(x^{\prime}, y^{\prime}\right) \mathrm{d} x^{\prime} \mathrm{d} y^{\prime} \\
&+\frac{1}{8} \int_{\Gamma \times \Gamma}\left\{\gamma_{b}^{-} u\left(x^{\prime}\right)-\gamma_{b}^{-} u\left(y^{\prime}\right)\right\}^{2} U_{0}\left(x^{\prime}, y^{\prime}\right) \mathrm{d} x^{\prime} \mathrm{d} y^{\prime}, \\
& \mathcal{F}=\left\{\begin{aligned}
\gamma_{a}^{+} u \in H^{1}(\Gamma) & \text { if } \kappa_{a}>0, \\
\gamma_{a}^{+} u \in H^{1 / 2}(\Gamma) & \text { if } \kappa_{a}=0, \\
\gamma_{b}^{-} u \in H^{1}(\Gamma) & \text { if } \kappa_{b}>0, \\
\gamma_{b}^{-} u \in H^{1 / 2}(\Gamma) & \text { if } \kappa_{b}=0
\end{aligned}\right\},
\end{aligned}
$$

where $E_{a b}=\Gamma \times(a, b), \Gamma_{a}=\Gamma \times\{a\}, \Gamma_{b}=\Gamma \times\{b\}, \gamma_{a}^{+}\left[\gamma_{b}^{-}\right]$stands for the trace operator on $\Gamma_{a}\left[\Gamma_{b}\right]$ from the domain $E_{a b}$, and $U_{0}$ is given by (5.49).

(iii) Let $m(\mathrm{~d} \xi)=I_{(-\infty, a)}(\xi) \mathrm{d} \xi+I_{(b, \infty)}(\xi) \mathrm{d} \xi$ and $\rho(\mathrm{d} \xi)=\kappa_{a} \delta_{a}(\mathrm{~d} \xi)+\kappa_{b} \delta_{b}(\mathrm{~d} \xi)$, where $-\infty<a<b<\infty$ and $\kappa_{a}, \kappa_{b}$ are nonnegative constants. Then the corresponding Dirichlet form in Theorem 5.1 is given by

$$
\begin{aligned}
\mathcal{E}(u, u)= & \frac{1}{2} \int_{E_{a} \cup E_{b}} \sum_{i=1}^{d}\left(\partial_{x^{i}} u\right)^{2} \mathrm{~d} x \\
& +\frac{\kappa_{a}}{2} \int_{\Gamma} \sum_{i=1}^{d-1}\left(\partial_{x^{i}} \gamma_{a}^{-} u\right)^{2} \mathrm{~d} x^{\prime}+\frac{\kappa_{b}}{2} \int_{\Gamma} \sum_{i=1}^{d-1}\left(\partial_{x^{i}} \gamma_{b}^{+} u\right)^{2} \mathrm{~d} x^{\prime} \\
& +\frac{1}{8} \int_{\Gamma \times \Gamma}\left\{\gamma_{a}^{-} u\left(x^{\prime}\right)-\gamma_{a}^{-} u\left(y^{\prime}\right)\right\}^{2} U_{a b}\left(x^{\prime}, y^{\prime}\right) \mathrm{d} x^{\prime} \mathrm{d} y^{\prime} \\
& +\frac{1}{8} \int_{\Gamma \times \Gamma}\left\{\gamma_{b}^{+} u\left(x^{\prime}\right)-\gamma_{b}^{+} u\left(y^{\prime}\right)\right\}^{2} U_{a b}\left(x^{\prime}, y^{\prime}\right) \mathrm{d} x^{\prime} \mathrm{d} y^{\prime} \\
& +\frac{1}{4} \int_{\Gamma \times \Gamma}\left\{\gamma_{a}^{-} u\left(x^{\prime}\right)-\gamma_{b}^{+} u\left(y^{\prime}\right)\right\}^{2} V_{a b}\left(x^{\prime}, y^{\prime}\right) \mathrm{d} x^{\prime} \mathrm{d} y^{\prime},
\end{aligned}
$$

where $E_{a}=\Gamma \times(-\infty, a), E_{b}=\Gamma \times(b, \infty), \Gamma_{a}=\Gamma \times\{a\}, \Gamma_{b}=\Gamma \times\{b\}, \gamma_{a}^{-}\left[\gamma_{b}^{+}\right]$ stands for the trace operator on $\Gamma_{a}\left[\Gamma_{b}\right]$ from the domain $E_{a}\left[E_{b}\right]$, and $U_{a b}\left(x^{\prime}, y^{\prime}\right)$ and $V_{a b}\left(x^{\prime}, y^{\prime}\right)$ are given by

$$
U_{a b}\left(x^{\prime}, y^{\prime}\right)=\int_{0}^{\infty}(2 \pi t)^{-(d-1) / 2} \mathrm{e}^{-\left|x^{\prime}-y^{\prime}\right|^{2} / 2 t} \frac{2}{b-a} \sum_{n=1}^{\infty}\left(\frac{n \pi}{b-a}\right)^{2} \mathrm{e}^{-(n \pi /(b-a))^{2} t / 2} \mathrm{~d} t
$$




$$
\begin{aligned}
= & 2^{-(d-5) / 2} \pi(b-a)^{-(d+3) / 2}\left|x^{\prime}-y^{\prime}\right|^{-(d-3) / 2} \\
& \times \sum_{n=1}^{\infty} n^{(d+1) / 2} K_{(d-3) / 2}\left(n \pi\left|x^{\prime}-y^{\prime}\right| /(b-a)\right), \\
V_{a b}\left(x^{\prime}, y^{\prime}\right)= & \int_{0}^{\infty}(2 \pi t)^{-(d-1) / 2} \mathrm{e}^{-\left|x^{\prime}-y^{\prime}\right|^{2} / 2 t} \frac{2}{b-a} \\
& \times \sum_{n=1}^{\infty}(-1)^{n-1}\left(\frac{n \pi}{b-a}\right)^{2} \mathrm{e}^{-(n \pi /(b-a))^{2} t / 2} \mathrm{~d} t \\
= & 2^{-(d-5) / 2} \pi(b-a)^{-(d+3) / 2}\left|x^{\prime}-y^{\prime}\right|^{-(d-3) / 2} \\
& \times \sum_{n=1}^{\infty}(-1)^{n-1} n^{(d+1) / 2} K_{(d-3) / 2}\left(n \pi\left|x^{\prime}-y^{\prime}\right| /(b-a)\right) .
\end{aligned}
$$

Note that $U_{a b}, V_{a b}$ satisfy (5.23) with $C_{2}=C_{4}=1 / 2$. Especially, if $d=2, U_{a b}, V_{a b}$ are reduced to

$$
\begin{aligned}
& U_{a b}\left(x^{\prime}, y^{\prime}\right)=\frac{2 \pi}{(b-a)^{2}} \mathrm{e}^{-\left|x^{\prime}-y^{\prime}\right| \pi /(b-a)}\left\{1-\mathrm{e}^{-\left|x^{\prime}-y^{\prime}\right| \pi /(b-a)}\right\}^{-2}, \\
& V_{a b}\left(x^{\prime}, y^{\prime}\right)=\frac{2 \pi}{(b-a)^{2}} \mathrm{e}^{-\left|x^{\prime}-y^{\prime}\right| \pi /(b-a)}\left\{1+\mathrm{e}^{-\left|x^{\prime}-y^{\prime}\right| \pi /(b-a)}\right\}^{-2},
\end{aligned}
$$

because of $K_{-1 / 2}(\xi)=\sqrt{\pi / 2 \xi} \mathrm{e}^{-\xi}$. By virtue of Lemma 5.2, it holds that for $\phi=\gamma_{a}^{-} u$ or $\gamma_{b}^{+} u$ with $u \in H^{1}\left(E_{a} \cup E_{b}\right)$,

$$
\begin{aligned}
& \iint_{\Gamma \times \Gamma}\left|\phi\left(x^{\prime}\right)-\phi\left(y^{\prime}\right)\right|^{2} U_{a b}\left(x^{\prime}, y^{\prime}\right) \mathrm{d} x^{\prime} \mathrm{d} y^{\prime} \leqslant c_{1}\|\phi\|_{H^{1 / 2}(\Gamma)}^{2} \\
& \quad \leqslant c_{2}\left\{\iint_{\Gamma \times \Gamma}\left|\phi\left(x^{\prime}\right)-\phi\left(y^{\prime}\right)\right|^{2} U_{a b}\left(x^{\prime}, y^{\prime}\right) \mathrm{d} x^{\prime} \mathrm{d} y^{\prime}+\|\phi\|_{L^{2}(\Gamma)}^{2}\right\},
\end{aligned}
$$

and further,

$$
\iint_{\Gamma \times \Gamma}\left|\gamma_{a}^{-} u\left(x^{\prime}\right)-\gamma_{b}^{+} u\left(y^{\prime}\right)\right|^{2} V_{a b}\left(x^{\prime}, y^{\prime}\right) \mathrm{d} x^{\prime} \mathrm{d} y^{\prime} \leqslant c_{3}\left\{\left\|\gamma_{a}^{-} u\right\|_{L^{2}(\Gamma)}^{2}+\left\|\gamma_{b}^{+} u\right\|_{L^{2}(\Gamma)}^{2}\right\},
$$

where $c_{i}(i=1,2,3)$ are positive constants. Therefore the domain $\mathcal{F}$ is given by

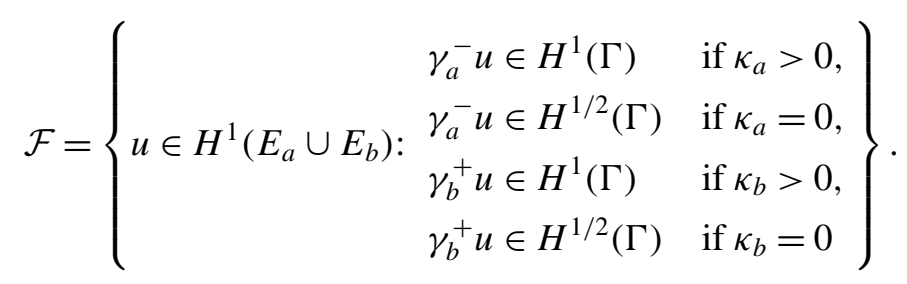

(iv) Let $m(\mathrm{~d} \xi)=\delta_{a}(\mathrm{~d} \xi)$ and $\rho(\mathrm{d} \xi)=\kappa_{a} \delta_{a}(\mathrm{~d} \xi)$, where $-\infty<a<\infty$ and $\kappa_{a}$ is a nonnegative constant. Then the corresponding Dirichlet space is given by 


$$
\begin{aligned}
& \mathcal{E}(u, u)=\frac{\kappa_{a}}{2} \int_{\Gamma} \sum_{i=1}^{d-1}\left(\partial_{x^{i}} u\left(x^{\prime}, a\right)\right)^{2} \mathrm{~d} x^{\prime} \\
& \quad+\frac{1}{4} \iint_{\Gamma \times \Gamma}\left\{u\left(x^{\prime}, a\right)-u\left(y^{\prime}, a\right)\right\}^{2} U_{0}\left(x^{\prime}, y^{\prime}\right) \mathrm{d} x^{\prime} \mathrm{d} y^{\prime}, \\
& \mathcal{F}= \begin{cases}H^{1}\left(\Gamma_{a}\right), & \text { if } \kappa_{a}>0, \\
H^{1 / 2}\left(\Gamma_{a}\right), & \text { if } \kappa_{a}=0,\end{cases}
\end{aligned}
$$

where $\Gamma_{a}=\Gamma \times\{a\}$ and $U_{0}$ is given by (5.49). The Dirichlet space corresponding to the case $\kappa_{a}=0$ is obtained in [7, Example 6.2.2]. The coefficient 1/4 in the right hand side of (5.52) is twice of $1 / 8$ in (6.2.33) of [7], because (5.52) is derived from the Brownian motion on the whole space $\mathbb{R}^{d}$ and (6.2.33) in [7] is derived from the reflecting Brownian motion on the half space.

(v) Let $\left\{a_{n}\right\}_{n=1}^{\infty}$ and $\left\{b_{n}\right\}_{n=0}^{\infty}$ be increasing sequences such that $-\infty<b_{0}<a_{1}<b_{1}<$ $\cdots<a_{n}<b_{n}<a_{n+1}<b_{n+1}<\cdots$, and $\lim _{n \rightarrow \infty} a_{n}=\lim _{n \rightarrow \infty} b_{n}=a_{*}<\infty$. Let

$$
m(\mathrm{~d} \xi)=\sum_{n=1}^{\infty} m_{n} I_{\left(b_{n-1}, a_{n}\right)}(\xi) \mathrm{d} \xi+m_{*} \delta_{a_{*}}(\mathrm{~d} \xi)
$$

where $m_{n}>0, m_{*} \geqslant 0$ and $\sum_{n=1}^{\infty} m_{n}\left(a_{n}-b_{n-1}\right)+m_{*}<\infty$. Hence $m$ is a finite measure on $\mathbb{R}$. In this case $S_{m}=\bigcup_{n=1}^{\infty}\left[b_{n-1}, a_{n}\right] \cup\left\{a_{*}\right\}$ and $\mathbb{R} \backslash S_{m}=\left(-\infty, b_{0}\right) \cup\left(\bigcup_{n=1}^{\infty}\left(a_{n}, b_{n}\right)\right) \cup$ $\left(a_{*}, \infty\right)$. Let

$$
\rho(\mathrm{d} \xi)=\sum_{n=1}^{\infty} \kappa_{a_{n}} \delta_{a_{n}}(\mathrm{~d} \xi)+\sum_{n=0}^{\infty} \kappa_{b_{n}} \delta_{b_{n}}(\mathrm{~d} \xi)+\kappa_{*} \delta_{a_{*}}(\mathrm{~d} \xi)
$$

where $\kappa_{a_{n}} \geqslant 0, \kappa_{b_{n}} \geqslant 0, \kappa_{*} \geqslant 0$, and $\sum_{n=1}^{\infty} \kappa_{a_{n}}+\sum_{n=0}^{\infty} \kappa_{b_{n}}+\kappa_{*}<\infty$. Hence $\rho$ is a finite measure on $\mathbb{R}$ and $\operatorname{supp}[\rho] \subset S_{m}$. Then (5.6) is reduced to

$$
\begin{aligned}
\mathcal{E}(u, u)= & \sum_{n=1}^{\infty} \frac{1}{2} \int_{E_{n}} \sum_{i=1}^{d}\left(\partial_{x^{i}} u(x)\right)^{2} \mathrm{~d} x+\sum_{n=1}^{\infty} \frac{\kappa_{a_{n}}}{2} \int_{\Gamma} \sum_{i=1}^{d-1}\left(\partial_{x^{i}} u\left(x^{\prime}, a_{n}\right)\right)^{2} \mathrm{~d} x^{\prime} \\
& +\sum_{n=0}^{\infty} \frac{\kappa_{b_{n}}}{2} \int_{\Gamma} \sum_{i=1}^{d-1}\left(\partial_{x^{i}} u\left(x^{\prime}, b_{n}\right)\right)^{2} \mathrm{~d} x^{\prime}+\frac{\kappa_{*}}{2} \int_{\Gamma} \sum_{i=1}^{d-1}\left(\partial_{x^{i}} u\left(x^{\prime}, a_{*}\right)\right)^{2} \mathrm{~d} x^{\prime} \\
& +\frac{1}{8} \sum_{n=1}^{\infty} \int_{\Gamma \times \Gamma}\left\{u\left(x^{\prime}, a_{n}\right)-u\left(y^{\prime}, a_{n}\right)\right\}^{2} U_{n}\left(x^{\prime}, y^{\prime}\right) \mathrm{d} x^{\prime} \mathrm{d} y^{\prime} \\
& +\frac{1}{8} \sum_{n=0}^{\infty} \int_{\Gamma \times \Gamma}\left\{u\left(x^{\prime}, b_{n}\right)-u\left(y^{\prime}, b_{n}\right)\right\}^{2} U_{n}\left(x^{\prime}, y^{\prime}\right) \mathrm{d} x^{\prime} \mathrm{d} y^{\prime} \\
& \left.+\frac{1}{8} \int_{\Gamma \times \Gamma} \int_{\{} u\left(x^{\prime}, a_{*}\right)-u\left(y^{\prime}, a_{*}\right)\right\}^{2} U_{0}\left(x^{\prime}, y^{\prime}\right) \mathrm{d} x^{\prime} \mathrm{d} y^{\prime}
\end{aligned}
$$




$$
+\frac{1}{4} \sum_{n=1}^{\infty} \iint_{\Gamma \times \Gamma}\left\{u\left(x^{\prime}, a_{n}\right)-u\left(y^{\prime}, b_{n}\right)\right\}^{2} V_{n}\left(x^{\prime}, y^{\prime}\right) \mathrm{d} x^{\prime} \mathrm{d} y^{\prime},
$$

for $\left.u \in C_{0}^{\infty}\left(\mathbb{R}^{d}\right)\right|_{\Omega_{\mu}}$, where $E_{n}=\Gamma \times\left(b_{n-1}, a_{n}\right), n=1,2, \ldots, U_{0}$ is defined by (5.49), $U_{n} \equiv U_{a_{n} b_{n}}$ is defined by (5.50) with $a=a_{n}, b=b_{n}$, and $V_{n} \equiv V_{a_{n} b_{n}}$ is defined by (5.51) with $a=a_{n}, b=b_{n}$ for each $n=1,2, \ldots$.

The domain $\mathcal{F}$ is the completion of $\left.C_{0}^{\infty}\left(\mathbb{R}^{d}\right)\right|_{\Omega_{\mu}}$ with respect to the norm $\mathcal{E}_{1}(\cdot, \cdot)^{1 / 2}$, where $\mathcal{E}_{1}(u, u)=\mathcal{E}(u, u)+(u, u)_{L^{2}\left(\Omega_{\mu}, \mu\right)}$. Therefore every $u \in \mathcal{F}$ possesses at least the following properties.

(v.1) If $m_{*}>0$, then $u\left(\cdot, a_{*}\right) \in H^{1}(\Gamma)$ or $u\left(\cdot, a_{*}\right) \in H^{1 / 2}(\Gamma)$ according to $\kappa_{*}>0$ or $\kappa_{*}=0$.

(v.2) $u \in H^{1}\left(E_{n}\right)$ for $n=1,2, \ldots$ and

$$
\|u\|_{(C)}^{2}:=\sum_{n=1}^{\infty}\left\{\int_{E_{n}} \sum_{i=1}^{d}\left(\partial_{x^{i}} u\right)^{2} \mathrm{~d} x+m_{n} \int_{E_{n}} u^{2} \mathrm{~d} x\right\}<\infty .
$$

(v.3) Both of $\gamma_{a_{n}}^{-} u$ and $\gamma_{b_{n-1}}^{+} u$ belong to $H^{1 / 2}(\Gamma)$ for $n=1,2, \ldots$, and

$$
\begin{aligned}
\|u\|_{(J)}^{2}:= & \sum_{n=1}^{\infty} \iint_{\Gamma \times \Gamma}\left\{\gamma_{a_{n}}^{-} u\left(x^{\prime}\right)-\gamma_{a_{n}}^{-} u\left(y^{\prime}\right)\right\}^{2} U_{n}\left(x^{\prime}, y^{\prime}\right) \mathrm{d} x^{\prime} \mathrm{d} y^{\prime} \\
& +\sum_{n=0}^{\infty} \iint_{\Gamma \times \Gamma}\left\{\gamma_{b_{n}}^{+} u\left(x^{\prime}\right)-\gamma_{b_{n}}^{+} u\left(y^{\prime}\right)\right\}^{2} U_{n}\left(x^{\prime}, y^{\prime}\right) \mathrm{d} x^{\prime} \mathrm{d} y^{\prime} \\
& +\sum_{n=1}^{\infty} \iint_{\Gamma \times \Gamma}\left\{\gamma_{a_{n}}^{-} u\left(x^{\prime}\right)-\gamma_{b_{n}}^{+} u\left(y^{\prime}\right)\right\}^{2} V_{n}\left(x^{\prime}, y^{\prime}\right) \mathrm{d} x^{\prime} \mathrm{d} y^{\prime}<\infty .
\end{aligned}
$$

(v.4) For each $n=1,2, \ldots, \gamma_{a_{n}}^{-} u \in H^{1}(\Gamma)$ if $\kappa_{a_{n}}>0$. For each $n=0,1,2, \ldots$, $\gamma_{b_{n}}^{+} u \in H^{1}(\Gamma)$ if $\kappa_{b_{n}}>0$. Further

$$
\sum_{n=1}^{\infty} \kappa_{a_{n}} \int_{\Gamma} \sum_{i=1}^{d-1}\left(\partial_{x^{i}} \gamma_{a_{n}}^{-} u\right)^{2} \mathrm{~d} x^{\prime}+\sum_{n=0}^{\infty} \kappa_{b_{n}} \int_{\Gamma} \sum_{i=1}^{d-1}\left(\partial_{x^{i}} \gamma_{b_{n}}^{+} u\right)^{2} \mathrm{~d} x^{\prime}<\infty .^{5}
$$

Here $\gamma_{a_{n}}^{-}$and $\gamma_{b_{n-1}}^{+}$are the trace operators on $\Gamma_{a_{n}}:=\Gamma \times\left\{a_{n}\right\}$ and $\Gamma_{b_{n-1}}:=\Gamma \times\left\{b_{n-1}\right\}$ from the domain $E_{n}$, respectively.

By means of above properties, it is easy to see that every $u \in \mathcal{F}$ satisfies the following identity and finiteness condition.

$$
\gamma_{a_{n}}^{-} u\left(x^{\prime}\right)-\gamma_{b_{n-1}}^{+} u\left(x^{\prime}\right)=\int_{b_{n-1}}^{a_{n}} \partial_{x^{d}} u\left(x^{\prime}, x^{d}\right) \mathrm{d} x^{d}, \quad \text { a.e. } x^{\prime} \in \Gamma .
$$

\footnotetext{
${ }^{5}$ We use the convention $0 \cdot \infty=0$ throughout this paper.
} 


$$
\begin{aligned}
& \sum_{n=1}^{\infty} \int_{b_{n-1}}^{a_{n}} \sum_{i=1}^{d}\left(\partial_{x^{i}} u\left(x^{\prime}, x^{d}\right)\right)^{2} \mathrm{~d} x^{d} \\
& \quad+\sum_{n=1}^{\infty} \int_{\Gamma}\left\{\gamma_{a_{n}}^{-} u\left(x^{\prime}\right)-\gamma_{a_{n}}^{-} u\left(y^{\prime}\right)\right\}^{2} U_{n}\left(x^{\prime}, y^{\prime}\right) \mathrm{d} y^{\prime} \\
& \quad+\sum_{n=0}^{\infty} \int_{\Gamma}\left\{\gamma_{b_{n}}^{+} u\left(x^{\prime}\right)-\gamma_{b_{n}}^{+} u\left(y^{\prime}\right)\right\}^{2} U_{n}\left(x^{\prime}, y^{\prime}\right) \mathrm{d} y^{\prime} \\
& \quad+\sum_{n=1}^{\infty} \int_{\Gamma}\left\{\gamma_{a_{n}}^{-} u\left(x^{\prime}\right)-\gamma_{b_{n}}^{+} u\left(y^{\prime}\right)\right\}^{2} V_{n}\left(x^{\prime}, y^{\prime}\right) \mathrm{d} y^{\prime}<\infty, \quad \text { a.e. } x^{\prime} \in \Gamma .
\end{aligned}
$$

Let us remark that, for every $u \in \mathcal{F}$, there exists the limit

$$
\gamma_{*} u\left(x^{\prime}\right):=\lim _{n \rightarrow \infty} \gamma_{\varepsilon_{n}} u\left(x^{\prime}\right), \quad \text { a.e. } x^{\prime} \in \Gamma,
$$

where $\varepsilon_{n}=a_{n}$ or $b_{n}, \gamma_{\varepsilon_{n}}=\gamma_{a_{n}}^{-}$if $\varepsilon_{n}=a_{n},=\gamma_{b_{n}}^{+}$if $\varepsilon_{n}=b_{n}$, for each $n \in \mathbb{N}$, and further it holds that

$$
\begin{aligned}
& \gamma_{*} u\left(x^{\prime}\right)=u\left(x^{\prime}, a_{*}\right), \quad \text { a.e. } x^{\prime} \in \Gamma, \quad \text { if } m_{*}>0, \\
& \left\|\gamma_{*} u\right\|_{L^{2}(\Gamma)} \leqslant c_{1}\left\{\|u\|_{(C)}+\|u\|_{(J)}+\left\|\gamma_{b_{0}}^{+} u\right\|_{L^{2}(\Gamma)}\right\},
\end{aligned}
$$

where $c_{1}$ is a positive constant independent of $u \in \mathcal{F}$. In order to prove above properties (5.56)-(5.58), it is enough to show that there is a positive constant $c_{2}$ such that, for $u \in \mathcal{F}, m, n=0,1,2, \ldots(m<n)$, and for a.e. $x^{\prime} \in \Gamma$,

$$
\begin{aligned}
\left|\gamma_{\varepsilon_{n}} u\left(x^{\prime}\right)-\gamma_{\varepsilon_{m}} u\left(x^{\prime}\right)\right|^{2} \leqslant & c_{2}\left\{\sum_{k=m}^{n-1} \int_{b_{k}}^{a_{k+1}}\left(\partial_{x^{d}} u\left(x^{\prime}, x^{d}\right)\right)^{2} \mathrm{~d} x^{d}\right. \\
& +\sum_{k=m}^{n} \int_{\Gamma}\left|\gamma_{a_{k}}^{-} u\left(x^{\prime}\right)-\gamma_{a_{k}}^{-} u\left(y^{\prime}\right)\right|^{2} U_{k}\left(x^{\prime}, y^{\prime}\right) \mathrm{d} y^{\prime} \\
& \left.+\sum_{k=m}^{n} \int_{\Gamma}\left|\gamma_{b_{k}}^{+} u\left(x^{\prime}\right)-\gamma_{a_{k}}^{-} u\left(y^{\prime}\right)\right|^{2} V_{k}\left(x^{\prime}, y^{\prime}\right) \mathrm{d} y^{\prime}\right\} .
\end{aligned}
$$

We give a proof of (5.59) in the case where $\varepsilon_{n}=a_{n}, \varepsilon_{m}=b_{m}$. Since

$$
\begin{aligned}
\gamma_{a_{n}}^{-} u\left(x^{\prime}\right)-\gamma_{b_{m}}^{+} u\left(x^{\prime}\right)= & \sum_{k=m}^{n-1}\left\{\gamma_{a_{k+1}}^{-} u\left(x^{\prime}\right)-\gamma_{b_{k}}^{+} u\left(x^{\prime}\right)\right\}+\sum_{k=m+1}^{n-1}\left\{\gamma_{b_{k}}^{+} u\left(x^{\prime}\right)-\gamma_{a_{k}}^{-} u\left(y_{k}^{\prime}\right)\right\} \\
& +\sum_{k=m+1}^{n-1}\left\{\gamma_{a_{k}}^{-} u\left(y_{k}^{\prime}\right)-\gamma_{a_{k}}^{-} u\left(x^{\prime}\right)\right\},
\end{aligned}
$$

for $y_{k}^{\prime} \in \Gamma(k=m+1, \ldots, n-1)$, by virtue of (5.54), we find that

$$
\left|\gamma_{a_{n}}^{-} u\left(x^{\prime}\right)-\gamma_{b_{m}}^{+} u\left(x^{\prime}\right)\right|^{2} \leqslant 3\left\{\sum_{k=m}^{n-1} \int_{b_{k}}^{a_{k+1}}\left(\partial_{x^{d}} u\left(x^{\prime}, x^{d}\right)\right)^{2} \mathrm{~d} x^{d} \cdot \sum_{k=m}^{n-1}\left(a_{k+1}-b_{k}\right)\right.
$$




$$
\begin{aligned}
& +\left(\sum_{k=m+1}^{n-1}\left|\gamma_{b_{k}}^{+} u\left(x^{\prime}\right)-\gamma_{a_{k}}^{-} u\left(y_{k}^{\prime}\right)\right|\right)^{2} \\
& \left.+\left(\sum_{k=m+1}^{n-1}\left|\gamma_{a_{k}}^{-} u\left(y_{k}^{\prime}\right)-\gamma_{a_{k}}^{-} u\left(x^{\prime}\right)\right|\right)^{2}\right\} .
\end{aligned}
$$

Put $A_{k}=\int_{\Gamma} V_{k}\left(x^{\prime}, y^{\prime}\right) \mathrm{d} y^{\prime}$, which is independent of $x^{\prime}$ because $V_{k}\left(x^{\prime}, y^{\prime}\right)$ is a function of $\left|x^{\prime}-y^{\prime}\right|$ by means of (5.51). By virtue of Lemma 5.2, $c_{3}:=\inf _{k \in \mathbb{N}, x^{\prime} \in \Gamma}\left(b_{k}-a_{k}\right) A_{k}>0$. Let $v_{k}\left(x^{\prime}, \mathrm{d} y^{\prime}\right)=A_{k}^{-1} V_{k}\left(x^{\prime}, y^{\prime}\right) \mathrm{d} y^{\prime}$. Integrating the second term of the right hand side of (5.60) by the product measure $\prod_{j=m+1}^{n-1} v_{j}\left(x^{\prime}, \mathrm{d} y_{j}^{\prime}\right)$ over the set $\Gamma^{n-m-1}$, we find that

$$
\begin{aligned}
\int_{\Gamma^{n-m-1}} & \left(\sum_{k=m+1}^{n-1}\left|\gamma_{b_{k}}^{+} u\left(x^{\prime}\right)-\gamma_{a_{k}}^{-} u\left(y_{k}^{\prime}\right)\right|\right)^{2} \prod_{j=m+1}^{n-1} v_{j}\left(x^{\prime}, \mathrm{d} y_{j}^{\prime}\right) \\
= & \sum_{k=m+1}^{n-1} \int_{\Gamma}\left|\gamma_{b_{k}}^{+} u\left(x^{\prime}\right)-\gamma_{a_{k}}^{-} u\left(y^{\prime}\right)\right|^{2} v_{k}\left(x^{\prime}, \mathrm{d} y^{\prime}\right) \\
& +\sum_{k \neq l} \int_{\Gamma}\left|\gamma_{b_{k}}^{+} u\left(x^{\prime}\right)-\gamma_{a_{k}}^{-} u\left(y^{\prime}\right)\right| v_{k}\left(x^{\prime}, \mathrm{d} y^{\prime}\right) \\
& \times \int_{\Gamma}\left|\gamma_{b_{l}}^{+} u\left(x^{\prime}\right)-\gamma_{a_{l}}^{-} u\left(z^{\prime}\right)\right| v_{l}\left(x^{\prime}, \mathrm{d} z^{\prime}\right) \\
\leqslant & \left\{\sum_{k=m+1}^{n-1}\left(\int_{\Gamma}\left|\gamma_{b_{k}}^{+} u\left(x^{\prime}\right)-\gamma_{a_{k}}^{-} u\left(y^{\prime}\right)\right|^{2} v_{k}\left(x^{\prime}, \mathrm{d} y^{\prime}\right)\right)^{1 / 2}\right\}^{2} \\
\leqslant & \sum_{k=m+1}^{n-1} \int_{\Gamma}\left|\gamma_{b_{k}}^{+} u\left(x^{\prime}\right)-\gamma_{a_{k}}^{-} u\left(y^{\prime}\right)\right|^{2} V_{k}\left(x^{\prime}, y^{\prime}\right) \mathrm{d} y^{\prime} \times \sum_{k=m+1}^{n-1} \frac{1}{A_{k}} \\
\leqslant & \frac{a_{*}-b_{0}}{c_{3}} \sum_{k=m+1}^{n-1} \int\left|\gamma_{b_{k}}^{+} u\left(x^{\prime}\right)-\gamma_{a_{k}}^{-} u\left(y^{\prime}\right)\right|^{2} V_{k}\left(x^{\prime}, y^{\prime}\right) \mathrm{d} y^{\prime} .
\end{aligned}
$$

Noting that $V_{k}\left(x^{\prime}, y^{\prime}\right) \leqslant U_{k}\left(x^{\prime}, y^{\prime}\right)$, and integrating both sides of (5.60) by the product measure $\prod_{j=m+1}^{n-1} v_{j}\left(x^{\prime}, \mathrm{d} y_{j}^{\prime}\right)$ over the set $\Gamma^{n-m-1}$, we obtain (5.59).

From above observation, it follows that the Dirichlet space $(\mathcal{E}, \mathcal{F})$ is given as follows:

$$
\begin{aligned}
\mathcal{E}(u, u)= & \sum_{n=1}^{\infty} \frac{1}{2} \int_{E_{n}} \sum_{i=1}^{d}\left(\partial_{x^{i}} u(x)\right)^{2} \mathrm{~d} x+\sum_{n=1}^{\infty} \frac{\kappa_{a_{n}}}{2} \int_{\Gamma} \sum_{i=1}^{d-1}\left(\partial_{x^{i}} \gamma_{a_{n}}^{-} u\left(x^{\prime}\right)\right)^{2} \mathrm{~d} x^{\prime} \\
& +\sum_{n=0}^{\infty} \frac{\kappa_{b_{n}}}{2} \int_{\Gamma} \sum_{i=1}^{d-1}\left(\partial_{x^{i}} \gamma_{b_{n}}^{+} u\left(x^{\prime}\right)\right)^{2} \mathrm{~d} x^{\prime}+\frac{\kappa_{*}}{2} \int_{\Gamma} \sum_{i=1}^{d-1}\left(\partial_{x^{i}} \gamma_{*} u\left(x^{\prime}\right)\right)^{2} \mathrm{~d} x^{\prime} \\
& +\frac{1}{8} \sum_{n=1}^{\infty} \iint_{\Gamma \times \Gamma}\left\{\gamma_{a_{n}}^{-} u\left(x^{\prime}\right)-\gamma_{a_{n}}^{-} u\left(y^{\prime}\right)\right\}^{2} U_{n}\left(x^{\prime}, y^{\prime}\right) \mathrm{d} x^{\prime} \mathrm{d} y^{\prime}
\end{aligned}
$$




$$
\begin{gathered}
+\frac{1}{8} \sum_{n=0}^{\infty} \iint_{\Gamma \times \Gamma}\left\{\gamma_{b_{n}}^{+} u\left(x^{\prime}\right)-\gamma_{b_{n}}^{+} u\left(y^{\prime}\right)\right\}^{2} U_{n}\left(x^{\prime}, y^{\prime}\right) \mathrm{d} x^{\prime} \mathrm{d} y^{\prime} \\
+\frac{1}{8} \iint_{\Gamma \times \Gamma}\left\{\gamma_{*} u\left(x^{\prime}\right)-\gamma_{*} u\left(y^{\prime}\right)\right\}^{2} U_{0}\left(x^{\prime}, y^{\prime}\right) \mathrm{d} x^{\prime} \mathrm{d} y^{\prime} \\
+\frac{1}{4} \sum_{n=1}^{\infty} \iint_{\Gamma \times \Gamma}\left\{\gamma_{a_{n}}^{-} u\left(x^{\prime}\right)-\gamma_{b_{n}}^{+} u\left(y^{\prime}\right)\right\}^{2} V_{n}\left(x^{\prime}, y^{\prime}\right) \mathrm{d} x^{\prime} \mathrm{d} y^{\prime}, \\
\mathcal{F}=\left\{\begin{array}{l}
u \text { satisfies (v.2), (v.3), (v.4), and further } \gamma_{*} u \in H^{1}(\Gamma) \\
\text { or } \gamma_{*} u \in H^{1 / 2}(\Gamma) \text { according to } \kappa_{*}>0 \text { or } \kappa_{*}=0
\end{array}\right\} .
\end{gathered}
$$

(vi) Let us consider the case where $E_{n}=\emptyset$ for every $n \in \mathbb{N}$ in (v). Namely, let $\left\{a_{n}\right\}_{n=1}^{\infty}$ be an increasing sequence with $\lim _{n \rightarrow \infty} a_{n}=a_{*} \in \mathbb{R}$. Let

$$
m(\mathrm{~d} \xi)=\sum_{n=1}^{\infty} m_{n} \delta_{a_{n}}(\mathrm{~d} \xi)+m_{*} \delta_{a_{*}}(\mathrm{~d} \xi),
$$

where $m_{n}>0, m_{*} \geqslant 0, \sum_{n=1}^{\infty} m_{n}+m_{*}<\infty$. Hence $m$ is a finite measure on $\mathbb{R}$. In this case $S_{m}=\bigcup_{n=1}^{\infty}\left\{a_{n}\right\} \cup\left\{a_{*}\right\}$ and $\mathbb{R} \backslash S_{m}=\left(-\infty, a_{1}\right) \cup\left(\bigcup_{n=1}^{\infty}\left(a_{n}, a_{n+1}\right)\right) \cup\left(a_{*}, \infty\right)$. Let

$$
\rho(\mathrm{d} \xi)=\sum_{n=1}^{\infty} \kappa_{n} \delta_{a_{n}}(\mathrm{~d} \xi)+\kappa_{*} \delta_{a_{*}}(\mathrm{~d} \xi)
$$

where $\kappa_{n} \geqslant 0, \kappa_{*} \geqslant 0, \sum_{n=1}^{\infty} \kappa_{n}+\kappa_{*}<\infty$. Hence $\rho$ is a finite measure on $\mathbb{R}$ and $\operatorname{supp}[\rho] \subset S_{m}$. Then (5.6) is reduced to

$$
\begin{aligned}
\mathcal{E}(u, u)= & \sum_{n=1}^{\infty} \frac{\kappa_{n}}{2} \int_{\Gamma} \sum_{i=1}^{d-1}\left(\partial_{x^{i}} u\left(x^{\prime}, a_{n}\right)\right)^{2} \mathrm{~d} x^{\prime}+\frac{\kappa_{*}}{2} \int_{\Gamma} \sum_{i=1}^{d-1}\left(\partial_{x^{i}} u\left(x^{\prime}, a_{*}\right)\right)^{2} \mathrm{~d} x^{\prime} \\
& +\frac{1}{8} \sum_{n=1}^{\infty} \iint_{\Gamma \times \Gamma}\left\{u\left(x^{\prime}, a_{n}\right)-u\left(y^{\prime}, a_{n}\right)\right\}^{2}\left(U_{n-1}\left(x^{\prime}, y^{\prime}\right)+U_{n}\left(x^{\prime}, y^{\prime}\right)\right) \mathrm{d} x^{\prime} \mathrm{d} y^{\prime} \\
& +\frac{1}{8} \iint_{\Gamma \times \Gamma}\left\{u\left(x^{\prime}, a_{*}\right)-u\left(y^{\prime}, a_{*}\right)\right\}^{2} U_{0}\left(x^{\prime}, y^{\prime}\right) \mathrm{d} x^{\prime} \mathrm{d} y^{\prime} \\
& +\frac{1}{4} \sum_{n=1}^{\infty} \int_{\Gamma \times \Gamma}\left\{u\left(x^{\prime}, a_{n}\right)-u\left(y^{\prime}, a_{n+1}\right)\right\}^{2} V_{n}\left(x^{\prime}, y^{\prime}\right) \mathrm{d} x^{\prime} \mathrm{d} y^{\prime},
\end{aligned}
$$

for $\left.u \in C_{0}^{\infty}\left(\mathbb{R}^{d}\right)\right|_{\Omega_{\mu}}$, where $U_{0}$ is defined by (5.49), $U_{n} \equiv U_{a_{n} a_{n+1}}$ is defined by (5.50) with $a=a_{n}, b=a_{n+1}$, and $V_{n} \equiv V_{a_{n} a_{n+1}}$ is defined by (5.51) with $a=a_{n}, b=a_{n+1}$ for each $n=1,2, \ldots$.

Since the domain $\mathcal{F}$ is the completion of $\left.C_{0}^{\infty}\left(\mathbb{R}^{d}\right)\right|_{\Omega_{\mu}}$ with respect to the norm $\mathcal{E}_{1}(\cdot, \cdot)^{1 / 2}$, every $u \in \mathcal{F}$ has the following properties. 
(vi.1) If $m_{*}>0$, then $u\left(\cdot, a_{*}\right) \in H^{1}(\Gamma)$ or $u\left(\cdot, a_{*}\right) \in H^{1 / 2}(\Gamma)$ according to $\kappa_{*}>0$ or $\kappa_{*}=0$.

(vi.2) $u\left(\cdot, a_{n}\right) \in H^{1 / 2}(\Gamma)$ for $n=1,2, \ldots$, and

$$
\begin{aligned}
\|u\|_{(J)}^{2}:= & \sum_{n=1}^{\infty} \iint_{\Gamma \times \Gamma}\left\{u\left(x^{\prime}, a_{n}\right)-u\left(y^{\prime}, a_{n}\right)\right\}^{2}\left(U_{n-1}\left(x^{\prime}, y^{\prime}\right)+U_{n}\left(x^{\prime}, y^{\prime}\right)\right) \mathrm{d} x^{\prime} \mathrm{d} y^{\prime} \\
& +\sum_{n=1}^{\infty} \iint_{\Gamma \times \Gamma}\left\{u\left(x^{\prime}, a_{n}\right)-u\left(y^{\prime}, a_{n+1}\right)\right\}^{2} V_{n}\left(x^{\prime}, y^{\prime}\right) \mathrm{d} x^{\prime} \mathrm{d} y^{\prime}<\infty .
\end{aligned}
$$

Further, by the same argument as in (v), we find that, for every $u \in \mathcal{F}$, there exists the limit

$$
\gamma_{*} u\left(x^{\prime}\right):=\lim _{n \rightarrow \infty} u\left(x^{\prime}, a_{n}\right), \quad \text { a.e. } x^{\prime} \in \Gamma,
$$

and

$$
\begin{aligned}
& \gamma_{*} u\left(x^{\prime}\right)=u\left(x^{\prime}, a_{*}\right), \quad \text { a.e. } x^{\prime} \in \Gamma, \quad \text { if } m_{*}>0, \\
& \left\|\gamma_{*} u\right\|_{L^{2}(\Gamma)} \leqslant c_{1}\left\{\|u\|_{(J)}+\left\|u\left(\cdot, a_{1}\right)\right\|_{L^{2}(\Gamma)}\right\},
\end{aligned}
$$

where $c_{1}$ is a positive constant independent of $u \in \mathcal{F}$. Thus the Dirichlet space $(\mathcal{E}, \mathcal{F})$ is given as follows.

$$
\begin{aligned}
& \mathcal{E}(u, u)= \sum_{n=1}^{\infty} \frac{\kappa_{n}}{2} \int_{\Gamma} \sum_{i=1}^{d-1}\left(\partial_{x^{i}} u\left(x^{\prime}, a_{n}\right)\right)^{2} \mathrm{~d} x^{\prime}+\frac{\kappa_{*}}{2} \int_{\Gamma} \sum_{i=1}^{d-1}\left(\partial_{x^{i}} \gamma_{*} u\left(x^{\prime}\right)\right)^{2} \mathrm{~d} x^{\prime} \\
&+\frac{1}{8} \sum_{n=1}^{\infty} \iint_{\Gamma \times \Gamma}\left\{u\left(x^{\prime}, a_{n}\right)-u\left(y^{\prime}, a_{n}\right)\right\}^{2}\left(U_{n-1}\left(x^{\prime}, y^{\prime}\right)+U_{n}\left(x^{\prime}, y^{\prime}\right)\right) \mathrm{d} x^{\prime} \mathrm{d} y^{\prime} \\
&+\frac{1}{8} \int_{\Gamma \times \Gamma}\left\{\gamma_{*} u\left(x^{\prime}\right)-\gamma_{*} u\left(y^{\prime}\right)\right\}^{2} U_{0}\left(x^{\prime}, y^{\prime}\right) \mathrm{d} x^{\prime} \mathrm{d} y^{\prime} \\
&+\frac{1}{4} \sum_{n=1}^{\infty} \iint_{\Gamma \times \Gamma}\left\{u\left(x^{\prime}, a_{n}\right)-u\left(y^{\prime}, a_{n+1}\right)\right\}^{2} V_{n}\left(x^{\prime}, y^{\prime}\right) \mathrm{d} x^{\prime} \mathrm{d} y^{\prime}, \\
& \mathcal{F}=\left\{\begin{array}{l}
u: \\
u \text { satisfies }\left(\text { vi.2), and further } \gamma_{*} u \in H^{1}(\Gamma)\right. \text { or } \\
\gamma_{*} u \in H^{1 / 2}(\Gamma) \text { according to } \kappa_{*}>0 \text { or } \kappa_{*}=0
\end{array}\right\} .
\end{aligned}
$$

(vii) Let $E$ be the triadic Cantor set, that is, $E=[0,1] \backslash \bigcup_{n=1}^{\infty} \bigcup_{e \in\{0,2\}^{n-1}}\left(a_{e}, b_{e}\right)$, where $a_{e}=\sum_{i=1}^{n-1} \varepsilon_{i} 3^{-i}+3^{-n}$ and $b_{e}=a_{e}+3^{-n}$, for $e=\left(\varepsilon_{1}, \ldots, \varepsilon_{n-1}\right) \in\{0,2\}^{n-1}$. Let $m$ be the Cantor measure, that is, a finite measure corresponding to the Cantor function. Then $S_{m}=E$. Let

$$
\rho(\mathrm{d} \xi)=\sum_{n=1}^{\infty} \sum_{e \in\{0,2\}^{n-1}}\left\{\kappa_{a_{e}} \delta_{a_{e}}(\mathrm{~d} \xi)+\kappa_{b_{e}} \delta_{b_{e}}(\mathrm{~d} \xi)\right\},
$$

where $\kappa_{a_{e}}$ and $\kappa_{b_{e}}$ are nonnegative constants and $\sum_{n=1}^{\infty} \sum_{e \in\{0,2\}^{n-1}}\left\{\kappa_{a_{e}}+\kappa_{b_{e}}\right\}<\infty$. Then $\rho$ is a finite measure and $\operatorname{supp}[\rho] \subset S_{m}$. Therefore (5.6) is reduced to 


$$
\begin{aligned}
\mathcal{E}(u, u)= & \frac{1}{2} \sum_{n=1}^{\infty} \sum_{e \in\{0,2\}^{n-1}} \kappa_{a_{e}} \int_{\Gamma} \sum_{i=1}^{d-1}\left(\partial_{x^{i}} u\left(x^{\prime}, a_{e}\right)\right)^{2} \mathrm{~d} x^{\prime} \\
& +\frac{1}{2} \sum_{n=1}^{\infty} \sum_{e \in\{0,2\}^{n-1}} \kappa_{b_{e}} \int_{\Gamma} \sum_{i=1}^{d-1}\left(\partial_{x^{i}} u\left(x^{\prime}, b_{e}\right)\right)^{2} \mathrm{~d} x^{\prime} \\
& +\frac{1}{8} \iint_{\Gamma \times \Gamma}\left\{u\left(x^{\prime}, 0\right)-u\left(y^{\prime}, 0\right)\right\}^{2} U_{0}\left(x^{\prime}, y^{\prime}\right) \mathrm{d} x^{\prime} \mathrm{d} y^{\prime} \\
& +\frac{1}{8} \int_{\Gamma \times \Gamma}\left\{u\left(x^{\prime}, 1\right)-u\left(y^{\prime}, 1\right)\right\}^{2} U_{0}\left(x^{\prime}, y^{\prime}\right) \mathrm{d} x^{\prime} \mathrm{d} y^{\prime} \\
& +\frac{1}{8} \sum_{n=1}^{\infty} \sum_{e \in\{0,2\}^{n-1}} \iint_{\Gamma \times \Gamma}\left\{u\left(x^{\prime}, a_{e}\right)-u\left(y^{\prime}, a_{e}\right)\right\}^{2} U_{e}\left(x^{\prime}, y^{\prime}\right) \mathrm{d} x^{\prime} \mathrm{d} y^{\prime} \\
& +\frac{1}{8} \sum_{n=1}^{\infty} \sum_{e \in\{0,2\}^{n-1}} \iint_{\Gamma \times \Gamma}\left\{u\left(x^{\prime}, b_{e}\right)-u\left(y^{\prime}, b_{e}\right)\right\}^{2} U_{e}\left(x^{\prime}, y^{\prime}\right) \mathrm{d} x^{\prime} \mathrm{d} y^{\prime} \\
& +\frac{1}{4} \sum_{n=1}^{\infty} \sum_{e \in\{0,2\}^{n-1}} \iint_{\Gamma \times \Gamma}\left\{u\left(x^{\prime}, a_{e}\right)-u\left(y^{\prime}, b_{e}\right)\right\}^{2} V_{e}\left(x^{\prime}, y^{\prime}\right) \mathrm{d} x^{\prime} \mathrm{d} y^{\prime},
\end{aligned}
$$

for $\left.u \in C_{0}^{\infty}\left(\mathbb{R}^{d}\right)\right|_{\Omega_{\mu}}$, where $U_{0}$ is defined by (5.49), $U_{e}$ is defined by (5.50) with $a=a_{e}, b=b_{e}$, and $V_{e}$ is defined by (5.51) with $a=a_{e}, b=b_{e}$. The domain $\mathcal{F}$ is the completion of $\left.C_{0}^{\infty}\left(\mathbb{R}^{d}\right)\right|_{\Omega_{\mu}}$ with respect to the norm $\left\{\mathcal{E}(\cdot, \cdot)+(\cdot, \cdot)_{L^{2}\left(\Omega_{\mu}, \mu\right)}\right\}^{1 / 2}$. Although we cannot determine the domain $\mathcal{F}$, we may say that

$\mathcal{F} \subset\left\{u \in L^{2}\left(\Omega_{\mu}, \mu\right): \sup _{N \in \mathbb{N}} \int_{\Gamma \times E \times \Gamma \times E}\left|u\left(x^{\prime}, x^{d}\right)-u\left(y^{\prime}, y^{d}\right)\right|^{2} v_{N}\left(\mathrm{~d} x^{\prime} \mathrm{d} x^{d} \mathrm{~d} y^{\prime} \mathrm{d} y^{d}\right)<\infty\right\}$,

where $v_{N}$ 's are Radon measures on $\Gamma \times E \times \Gamma \times E$ defined by

$$
\begin{aligned}
& v_{N}\left(\mathrm{~d} x^{\prime} \mathrm{d} x^{d} \mathrm{~d} y^{\prime} \mathrm{d} y^{d}\right) \\
& =U_{0}\left(x^{\prime}, y^{\prime}\right)\left\{\mathrm{d} x^{\prime} \delta_{0}\left(\mathrm{~d} x^{d}\right) \mathrm{d} y^{\prime} \delta_{0}\left(\mathrm{~d} y^{d}\right)+\mathrm{d} x^{\prime} \delta_{1}\left(\mathrm{~d} x^{d}\right) \mathrm{d} y^{\prime} \delta_{1}\left(\mathrm{~d} y^{d}\right)\right\} \\
& \quad+\sum_{n=1}^{N} \sum_{e \in\{0,2\}^{n-1}}\left\{U _ { e } ( x ^ { \prime } , y ^ { \prime } ) \left\{\mathrm{~d} x^{\prime} \delta_{a_{e}}\left(\mathrm{~d} x^{d}\right) \mathrm{d} y^{\prime} \delta_{a_{e}}\left(\mathrm{~d} y^{d}\right)\right.\right. \\
& \left.\left.\quad+\mathrm{d} x^{\prime} \delta_{b_{e}}\left(\mathrm{~d} x^{d}\right) \mathrm{d} y^{\prime} \delta_{b_{e}}\left(\mathrm{~d} y^{d}\right)\right\}+V_{e}\left(x^{\prime}, y^{\prime}\right) \mathrm{d} x^{\prime} \delta_{a_{e}}\left(\mathrm{~d} x^{d}\right) \mathrm{d} y^{\prime} \delta_{b_{e}}\left(\mathrm{~d} y^{d}\right)\right\} .
\end{aligned}
$$

\section{The partial differential equation for the limit process}

Let $\left\{a_{k}\right\}_{k=-\infty}^{\infty}$ and $\left\{b_{k}\right\}_{k=-\infty}^{\infty}$ be sequences such that $a_{k}<b_{k}<a_{k+1}<b_{k+1}, k=$ $0, \pm 1, \pm 2, \ldots$, and $\lim _{k \rightarrow-\infty} a_{k}=\lim _{k \rightarrow-\infty} b_{k}=-\infty, \lim _{k \rightarrow \infty} a_{k}=\lim _{k \rightarrow \infty} b_{k}=\infty$. Let $m(\mathrm{~d} \xi)=\sum_{k=-\infty}^{\infty} m_{k} I_{\left(b_{k}, a_{k+1}\right)}(\xi) \mathrm{d} \xi$ and $\rho(\mathrm{d} \xi)=\sum_{k=-\infty}^{\infty}\left\{\kappa_{a_{k}} \delta_{a_{k}}(\mathrm{~d} \xi)+\kappa_{b_{k}} \delta_{b_{k}}(\mathrm{~d} \xi)\right\}$, where $m_{k}$ 's are positive constants, and $\kappa_{a_{k}}$ 's and $\kappa_{b_{k}}$ 's are nonnegative constants. 
$m$ and $\rho$ are Radon measures on $\mathbb{R}$, $\operatorname{supp}[\rho] \subset S_{m}=\bigcup_{k=-\infty}^{\infty}\left[b_{k}, a_{k+1}\right]$, and $\mathbb{R} \backslash S_{m}=$ $\bigcup_{k=-\infty}^{\infty}\left(a_{k}, b_{k}\right)$. In view of Theorem 5.1, the corresponding Dirichlet form is given by

$$
\begin{aligned}
\mathcal{E}(u, u)= & \sum_{k=-\infty}^{\infty} \frac{1}{2} \int_{E_{k}}\left\{\sum_{i, j=1}^{d-1} a^{i j}\left(x^{\prime}\right) \partial_{x^{i}} u(x) \partial_{x^{j}} u(x)+a^{d d}\left(x^{d}\right) \partial_{x^{d}} u(x) \partial_{x^{d}} u(x)\right\} \mathrm{d} x \\
& +\sum_{k=-\infty}^{\infty} \frac{\kappa_{a_{k}}}{2} \int_{\Gamma} \sum_{i, j=1}^{d-1} a^{i j}\left(x^{\prime}\right) \partial_{x^{i}} \gamma_{a_{k}}^{-} u\left(x^{\prime}\right) \partial_{x^{j}} \gamma_{a_{k}}^{-} u\left(x^{\prime}\right) \mathrm{d} x^{\prime} \\
& +\sum_{k=-\infty}^{\infty} \frac{\kappa_{b_{k}}}{2} \int_{\Gamma} \sum_{i, j=1}^{d-1} a^{i j}\left(x^{\prime}\right) \partial_{x^{i}} \gamma_{b_{k}}^{+} u\left(x^{\prime}\right) \partial_{x^{j}} \gamma_{b_{k}}^{+} u\left(x^{\prime}\right) \mathrm{d} x^{\prime} \\
& +\frac{1}{8} \sum_{k=-\infty}^{\infty} \iint_{\Gamma \times \Gamma}\left\{\gamma_{a_{k}}^{-} u\left(x^{\prime}\right)-\gamma_{a_{k}}^{-} u\left(y^{\prime}\right)\right\}^{2} U_{k}\left(x^{\prime}, y^{\prime}\right) \mathrm{d} x^{\prime} \mathrm{d} y^{\prime} \\
& +\frac{1}{8} \sum_{k=-\infty}^{\infty} \iint_{\Gamma \times \Gamma}\left\{\gamma_{b_{k}}^{+} u\left(x^{\prime}\right)-\gamma_{b_{k}}^{+} u\left(y^{\prime}\right)\right\}^{2} U_{k}\left(x^{\prime}, y^{\prime}\right) \mathrm{d} x^{\prime} \mathrm{d} y^{\prime} \\
& +\frac{1}{4} \sum_{k=-\infty}^{\infty} \iint_{\Gamma \times \Gamma}\left\{\gamma_{a_{k}}^{-} u\left(x^{\prime}\right)-\gamma_{b_{k}}^{+} u\left(y^{\prime}\right)\right\}^{2} V_{k}\left(x^{\prime}, y^{\prime}\right) \mathrm{d} x^{\prime} \mathrm{d} y^{\prime},
\end{aligned}
$$

where $E_{k}=\Gamma \times\left(b_{k}, a_{k+1}\right), \Gamma_{a_{k}}=\Gamma \times\left\{a_{k}\right\}, \Gamma_{b_{k}}=\Gamma \times\left\{b_{k}\right\}, \gamma_{a_{k}^{-}}\left[\gamma_{b_{k}}^{+}\right]$is the trace operator on $\Gamma_{a_{k}}\left[\Gamma_{b_{k}}\right]$ from $E_{k-1}\left[E_{k}\right]$, and $U_{k}, V_{k}$ are given by (5.13) and (5.17). The domain $\mathcal{F}$ is the space of all measurable functions $u$ defined on $E:=\bigcup_{k=-\infty}^{\infty} E_{k}$ satisfying the following properties.

$(\mathcal{F} .1) u \in H^{1}\left(E_{k}\right)$ for $k=0, \pm 1, \pm 2, \ldots$, and

$$
\sum_{k=-\infty}^{\infty}\left\{\int_{E_{k}} \sum_{i=1}^{d}\left(\partial_{x^{i}} u\right)^{2} \mathrm{~d} x+m_{k} \int_{E_{k}} u^{2} \mathrm{~d} x\right\}<\infty .
$$

$(\mathcal{F} .2)$ For each $k=0, \pm 1, \pm 2, \ldots, \gamma_{a_{k}}^{-} u \in H^{1}(\Gamma)$ or $H^{1 / 2}(\Gamma)$ according to $\kappa_{a_{k}}>0$ or $\kappa_{a_{k}}=0$, and $\gamma_{b_{k}}^{+} u \in H^{1}(\Gamma)$ or $H^{1 / 2}(\Gamma)$ according to $\kappa_{b_{k}}>0$ or $\kappa_{b_{k}}=0$. Further

$$
\begin{aligned}
& \sum_{k=-\infty}^{\infty} \kappa_{a_{k}} \int_{\Gamma} \sum_{i=1}^{d-1}\left(\partial_{x^{i}} \gamma_{a_{k}}^{-} u\right)^{2} \mathrm{~d} x^{\prime}+\sum_{k=-\infty}^{\infty} \kappa_{b_{k}} \int_{\Gamma} \sum_{i=1}^{d-1}\left(\partial_{x^{i}} \gamma_{b_{k}}^{+} u\right)^{2} \mathrm{~d} x^{\prime} \\
& \quad+\sum_{k=-\infty}^{\infty} \iint_{\Gamma \times \Gamma}\left\{\gamma_{a_{k}}^{-} u\left(x^{\prime}\right)-\gamma_{a_{k}}^{-} u\left(y^{\prime}\right)\right\}^{2} U_{k}\left(x^{\prime}, y^{\prime}\right) \mathrm{d} x^{\prime} \mathrm{d} y^{\prime} \\
& \quad+\sum_{k=-\infty}^{\infty} \iint_{\Gamma \times \Gamma}\left\{\gamma_{b_{k}}^{+} u\left(x^{\prime}\right)-\gamma_{b_{k}}^{+} u\left(y^{\prime}\right)\right\}^{2} U_{k}\left(x^{\prime}, y^{\prime}\right) \mathrm{d} x^{\prime} \mathrm{d} y^{\prime} \\
& \quad+\sum_{k=-\infty}^{\infty} \int_{\Gamma \times \Gamma}\left\{\gamma_{a_{k}}^{-} u\left(x^{\prime}\right)-\gamma_{b_{k}}^{+} u\left(y^{\prime}\right)\right\}^{2} V_{k}\left(x^{\prime}, y^{\prime}\right) \mathrm{d} x^{\prime} \mathrm{d} y^{\prime}<\infty .
\end{aligned}
$$


Put $\mathcal{E}_{\lambda}(u, v)=\mathcal{E}(u, v)+\lambda(u, v)_{L^{2}(E, \mu)}$ for $\lambda>0$. By virtue of the general theory, for each $f \in L^{2}(E, \mu)$ and $\lambda>0$, there exists a unique element $u$ of $\mathcal{F}$ denoted by $G_{\lambda} f$ such that

$$
\mathcal{E}_{\lambda}(u, v)=(f, v)_{L^{2}(E, \mu)}, \quad v \in \mathcal{F} .
$$

It is known that $\left\{G_{\lambda}, \lambda>0\right\}$ is Markovian in the sense that $0 \leqslant \lambda G_{\lambda} f \leqslant 1$ whenever $0 \leqslant f \leqslant 1$ ([7]). Hence it is defined as a bounded linear operator on $C(\bar{E})$, where $\bar{E}=\bigcup_{k=-\infty}^{\infty} \bar{E}_{k}$ with $\bar{E}_{k}=E_{k} \cup \Gamma_{a_{k+1}} \cup \Gamma_{b_{k}}$. We pose the following assumption on the sequences $\left\{a_{k}\right\}_{k=-\infty}^{\infty}$ and $\left\{b_{k}\right\}_{k=-\infty}^{\infty}$.

$$
\begin{aligned}
\sum_{0 \leqslant k<\infty} m_{k}\left(a_{k+1}-b_{k}\right)^{2} & =\infty, \\
\sum_{-\infty<k<0} m_{k}\left(a_{k+1}-b_{k}\right)^{2} & =\infty,
\end{aligned}
$$

which imply that (4.5) and (4.6) are satisfied. Therefore we can derive the following assertion from Theorem 4.3.

THEOREM 6.1. - Under the conditions (6.2) and (6.3) it holds that

$$
G_{\lambda}(\widehat{C}(\bar{E})) \subset \widehat{C}(\bar{E}), \quad \lambda>0 .
$$

We are going to observe the assertion of Theorem 6.1 from a point of view of partial differential equation theory. In order to proceed our argument, we need the following assumption.

$$
a^{i j} \in C^{1, \alpha}(\Gamma), \quad i, j=1, \ldots, d-1,
$$

for an $\alpha \in(0,1]$. Here $C^{1, \alpha}(\Gamma)$ is the space of all continuously differentiable functions on $\Gamma$ with bounded derivatives and with the finite norm

$$
\|f\|_{1, \alpha}=\sum_{|r|=0,1}\left\|\partial^{r} f\right\|+\sum_{|r|=1}\left[\partial^{r} f\right]_{\alpha}
$$

where

$$
\begin{aligned}
\|f\| & =\sup _{x \in \Gamma}|f(x)|, \quad[f]_{\alpha}=\sup _{x, y \in \Gamma} \frac{|f(x)-f(y)|}{|x-y|^{\alpha}}, \\
\partial^{r} & =\left(\partial_{x^{1}}\right)^{r_{1}}\left(\partial_{x^{2}}\right)^{r_{2}} \cdots\left(\partial_{x^{d}}\right)^{r_{d}}, \quad r=\left(r_{1}, r_{2}, \ldots, r_{d}\right), \\
|r| & =r_{1}+r_{2}+\cdots+r_{d} .
\end{aligned}
$$

In the following, we fix a nonnegative element $\chi \in C_{0}^{\infty}(\Gamma)$ such that $\chi\left(y^{\prime}\right)=1$ for $\left|y^{\prime}\right| \leqslant 1 / 2, \chi\left(y^{\prime}\right)=0$ for $\left|y^{\prime}\right| \geqslant 1$, and $\chi\left(-y^{\prime}\right)=\chi\left(y^{\prime}\right)$.

PROPOSITION 6.2. - Under the assumption (6.4), there exists the limit

$$
b_{k}^{i}\left(x^{\prime}\right)=\lim _{\varepsilon \downarrow 0} \int_{\Gamma \backslash \Delta_{\varepsilon}^{x^{\prime}}}\left(y^{i}-x^{i}\right) \chi\left(y^{\prime}-x^{\prime}\right) U_{k}\left(x^{\prime}, y^{\prime}\right) \mathrm{d} y^{\prime}, \quad x^{\prime} \in \Gamma,
$$

for each $i=1,2, \ldots, d-1$, and $k=0, \pm 1, \pm 2, \ldots$, where $\Delta_{\varepsilon}^{x^{\prime}}=\left\{y^{\prime} \in \Gamma:\left|x^{\prime}-y^{\prime}\right|<\varepsilon\right\}$. 
In order to prove Proposition 6.2, we need some estimates of $p^{\prime}\left(t, x^{\prime}, y^{\prime}\right)$. We summarize them first of all. Following the parametric method, we find that $p^{\prime}\left(t, x^{\prime}, y^{\prime}\right)$ is represented as

$$
p^{\prime}\left(t, x^{\prime}, y^{\prime}\right)=\bar{q}^{y^{\prime}}\left(t, x^{\prime}, y^{\prime}\right)+\widetilde{q}\left(t, x^{\prime}, y^{\prime}\right)
$$

where

$$
\bar{q}^{z^{\prime}}\left(t, x^{\prime}, y^{\prime}\right)=\left(\operatorname{det} a\left(z^{\prime}\right)\right)^{1 / 2}(2 \pi t)^{-(d-1) / 2} \exp \left\{-\frac{1}{2 t}\left(x^{\prime}-y^{\prime}\right) a\left(z^{\prime}\right)^{t}\left(x^{\prime}-y^{\prime}\right)\right\},
$$

$a\left(z^{\prime}\right)=\left(a_{i j}\left(z^{\prime}\right)\right)=\left(a^{i j}\left(z^{\prime}\right)\right)^{-1}$, and $\widetilde{q}\left(t, x^{\prime}, y^{\prime}\right)$ is the remainder term.

LEMMA 6.3. - There are positive constants $C_{13}$ and $C_{14}$ such that

$$
\left|\widetilde{q}\left(t, x^{\prime}, y^{\prime}\right)\right| \leqslant C_{13} t^{-(d-1-\alpha) / 2} \mathrm{e}^{-C_{14}\left|x^{\prime}-y^{\prime}\right|^{2} / t}, \quad 0<t<\infty, x^{\prime}, y^{\prime} \in \Gamma .
$$

Proof. - In the following, $c_{1}, c_{2}$, etc. stand for positive constants independent of variables. Let $x^{\prime}, y^{\prime}, z^{\prime} \in \Gamma$. By means of (6.7),

$$
\left|\bar{q}^{z^{\prime}}\left(t, x^{\prime}, y^{\prime}\right)\right| \leqslant c_{1} t^{-(d-1) / 2} \mathrm{e}^{-c_{2}\left|x^{\prime}-y^{\prime}\right|^{2} / t}, \quad 0<t<\infty .
$$

Combining this with (4.31), we get

$$
\left|\widetilde{q}\left(t, x^{\prime}, y^{\prime}\right)\right| \leqslant c_{3} t^{-(d-1) / 2} \mathrm{e}^{-c_{4}\left|x^{\prime}-y^{\prime}\right|^{2} / t}, \quad 0<t<\infty .
$$

In $[13$, p. $378,(13.5)]$, the following estimate is already obtained.

$$
\left|\widetilde{q}\left(t, x^{\prime}, y^{\prime}\right)\right| \leqslant c_{5} t^{-(d-1-\alpha) / 2} \mathrm{e}^{-c_{6}\left|x^{\prime}-y^{\prime}\right|^{2} / t}, \quad 0<t \leqslant T,
$$

where $T>0$ is fixed arbitrarily. This estimate coupled with (6.10) implies (6.8).

Proof of Proposition 6.2. - We put

$$
W_{k}\left(y^{\prime} ; z^{\prime}\right)=\left(\operatorname{det} a\left(z^{\prime}\right)\right)^{1 / 2} \int_{0}^{\infty}(2 \pi t)^{-(d-1) / 2} \exp \left\{-\frac{1}{2 t} y^{\prime} a\left(z^{\prime}\right)^{t} y^{\prime}\right\} \alpha_{k}(t) \mathrm{d} t,
$$

for $y^{\prime}, z^{\prime} \in \Gamma$. We note that there is a positive constant $C_{15}$ such that

$$
\left|W_{k}\left(y^{\prime} ; x^{\prime}\right)-W_{k}\left(y^{\prime} ; z^{\prime}\right)\right| \leqslant C_{15}\left|x^{\prime}-z^{\prime}\right|\left|y^{\prime}\right|^{-d},
$$

for every $x^{\prime}, y^{\prime}, z^{\prime} \in \Gamma, k=0, \pm 1, \pm 2, \ldots$ Indeed, noting (6.4), we see that

$$
\begin{aligned}
\left|W_{k}\left(y^{\prime} ; x^{\prime}\right)-W_{k}\left(y^{\prime} ; z^{\prime}\right)\right| & =\left|\int_{0}^{1} \partial_{s} W_{k}\left(y^{\prime} ; x^{\prime}+s\left(z^{\prime}-x^{\prime}\right)\right) \mathrm{d} s\right| \\
& \leqslant\left|x^{\prime}-z^{\prime}\right| \int_{0}^{1} \mid \nabla_{\eta^{\prime}} W_{k}\left(y^{\prime} ; \eta^{\prime}\right) \|_{\eta^{\prime}=x^{\prime}+s\left(z^{\prime}-x^{\prime}\right)} \mathrm{d} s
\end{aligned}
$$




$$
\begin{aligned}
& \leqslant c_{1}\left|x^{\prime}-z^{\prime}\right|\left\{\int_{0}^{\infty} t^{-(d-1) / 2} \mathrm{e}^{-c_{2}\left|y^{\prime}\right|^{2} / t} \alpha_{k}(t) \mathrm{d} t\right. \\
& \left.+\left|y^{\prime}\right|^{2} \int_{0}^{\infty} t^{-(d+1) / 2} \mathrm{e}^{-c_{2}\left|y^{\prime}\right|^{2} / t} \alpha_{k}(t) \mathrm{d} t\right\},
\end{aligned}
$$

for some positive constants $c_{1}$ and $c_{2}$. Combining this with (5.24) and (5.25), we get (6.12).

By virtue of (6.6),

$$
\begin{aligned}
U_{k}\left(x^{\prime}, y^{\prime}\right) & =\int_{0}^{\infty} \bar{q}^{y^{\prime}}\left(t, x^{\prime}, y^{\prime}\right) \alpha_{k}(t) \mathrm{d} t+\int_{0}^{\infty} \widetilde{q}\left(t, x^{\prime}, y^{\prime}\right) \alpha_{k}(t) \mathrm{d} t \\
& \equiv U_{k, 1}\left(x^{\prime}, y^{\prime}\right)+U_{k, 2}\left(x^{\prime}, y^{\prime}\right)
\end{aligned}
$$

From (6.11),

$$
U_{k, 1}\left(x^{\prime}, y^{\prime}\right)=W_{k}\left(y^{\prime}-x^{\prime} ; y^{\prime}\right) .
$$

Noting that $\int_{\Gamma \backslash \Delta_{\varepsilon}^{x^{\prime}}}\left(y^{i}-x^{i}\right) \chi\left(y^{\prime}-x^{\prime}\right) W_{k}\left(y^{\prime}-x^{\prime} ; x^{\prime}\right) \mathrm{d} y^{\prime}=0$, we have

$$
\begin{aligned}
& \int_{\Gamma \backslash \Delta_{\varepsilon}^{x^{\prime}}}\left(y^{i}-x^{i}\right) \chi\left(y^{\prime}-x^{\prime}\right) U_{k, 1}\left(x^{\prime}, y^{\prime}\right) \mathrm{d} y^{\prime} \\
& \quad=\int_{\Gamma \backslash \Delta_{\varepsilon}^{x^{\prime}}}\left(y^{i}-x^{i}\right) \chi\left(y^{\prime}-x^{\prime}\right)\left\{W_{k}\left(y^{\prime}-x^{\prime} ; y^{\prime}\right)-W_{k}\left(y^{\prime}-x^{\prime} ; x^{\prime}\right)\right\} \mathrm{d} y^{\prime} .
\end{aligned}
$$

By virtue of (6.12),

$$
\left|W_{k}\left(y^{\prime}-x^{\prime} ; y^{\prime}\right)-W_{k}\left(y^{\prime}-x^{\prime} ; x^{\prime}\right)\right| \leqslant C_{15}\left|x^{\prime}-y^{\prime}\right|^{-d+1} .
$$

Therefore there exists the following limit:

$$
\begin{aligned}
b_{k, 1}^{i}\left(x^{\prime}\right) & :=\lim _{\varepsilon \downarrow 0} \int_{\Gamma \backslash \Delta_{\varepsilon}^{x^{\prime}}}\left(y^{i}-x^{i}\right) \chi\left(y^{\prime}-x^{\prime}\right) U_{k, 1}\left(x^{\prime}, y^{\prime}\right) \mathrm{d} y^{\prime} \\
& =\int_{\Gamma}\left(y^{i}-x^{i}\right) \chi\left(y^{\prime}-x^{\prime}\right)\left\{W_{k}\left(y^{\prime}-x^{\prime} ; y^{\prime}\right)-W_{k}\left(y^{\prime}-x^{\prime} ; x^{\prime}\right)\right\} \mathrm{d} y^{\prime} \\
& =\int_{\Gamma} y^{i} \chi\left(y^{\prime}\right)\left\{W_{k}\left(y^{\prime} ; x^{\prime}+y^{\prime}\right)-W_{k}\left(y^{\prime} ; x^{\prime}\right)\right\} \mathrm{d} y^{\prime} .
\end{aligned}
$$

By means of (6.8), (5.24) and (5.25),

$$
\left|U_{k, 2}\left(x^{\prime}, y^{\prime}\right)\right| \leqslant c_{1} \int_{0}^{\infty} t^{-(d+2-\alpha) / 2} \mathrm{e}^{-c_{2}\left|x^{\prime}-y^{\prime}\right|^{2} / t} \mathrm{~d} t \leqslant c_{3}\left|x^{\prime}-y^{\prime}\right|^{-(d-\alpha)}
$$

for some positive constants $c_{i}(i=1,2,3)$. Therefore there exists the following limit: 


$$
\begin{aligned}
b_{k, 2}^{i}\left(x^{\prime}\right) & :=\lim _{\varepsilon \downarrow 0} \int_{\Gamma \backslash \Delta_{\varepsilon}^{x^{\prime}}}\left(y^{i}-x^{i}\right) \chi\left(y^{\prime}-x^{\prime}\right) U_{k, 2}\left(x^{\prime}, y^{\prime}\right) \mathrm{d} y^{\prime} \\
& =\int_{\Gamma}\left(y^{i}-x^{i}\right) \chi\left(y^{\prime}-x^{\prime}\right) U_{k, 2}\left(x^{\prime}, y^{\prime}\right) \mathrm{d} y^{\prime} .
\end{aligned}
$$

Thus there exists the limit $b_{k}^{i}$ defined by (6.5).

We define the following operators.

$$
\begin{aligned}
\mathcal{A} u(x)= & \frac{1}{2}\left\{\sum_{i, j=1}^{d-1} \partial_{x^{i}}\left(a^{i j}\left(x^{\prime}\right) \partial_{x^{j}} u(x)\right)+\partial_{x^{d}}\left(a^{d d}\left(x^{d}\right) \partial_{x^{d}} u(x)\right)\right\}, \\
\partial_{n_{a}^{-}} u\left(x^{\prime}\right)= & \frac{1}{2} a^{d d}(a) \partial_{x^{d}} u\left(x^{\prime}, a\right), \quad \partial_{n_{b}^{+}} u\left(x^{\prime}\right)=-\frac{1}{2} a^{d d}(b) \partial_{x^{d}} u\left(x^{\prime}, b\right), \\
\widetilde{\mathcal{A}}_{\xi}^{ \pm} u\left(x^{\prime}\right)= & \frac{\kappa_{\xi}}{2} \sum_{i, j=1}^{d-1} \partial_{x^{i}}\left(a^{i j}\left(x^{\prime}\right) \partial_{x^{j}} \gamma_{\xi}^{ \pm} u\left(x^{\prime}\right)\right), \\
\widetilde{\mathcal{B}}_{\xi}^{ \pm} u\left(x^{\prime}\right)= & \frac{1}{4} \int_{\Gamma}\left\{\gamma_{\xi}^{ \pm} u\left(x^{\prime}+y^{\prime}\right)-\gamma_{\xi}^{ \pm} u\left(x^{\prime}\right)-\nabla_{x^{\prime}} \gamma_{\xi}^{ \pm} u\left(x^{\prime}\right) \cdot y^{\prime} \chi\left(y^{\prime}\right)\right\} \\
& \times U_{k}\left(x^{\prime}, x^{\prime}+y^{\prime}\right) \mathrm{d} y^{\prime}+\frac{1}{4} \sum_{i=1}^{d-1} b_{k}^{i}\left(x^{\prime}\right) \partial_{x^{i}} \gamma_{\xi}^{ \pm} u\left(x^{\prime}\right), \\
\widetilde{\mathcal{C}}_{a_{k}}^{+-} u\left(x^{\prime}\right)= & \frac{1}{4} \int_{\Gamma}\left\{\gamma_{b_{k}}^{+} u\left(x^{\prime}+y^{\prime}\right)-\gamma_{a_{k}}^{-} u\left(x^{\prime}\right)\right\} V_{k}\left(x^{\prime}, x^{\prime}+y^{\prime}\right) \mathrm{d} y^{\prime}, \\
\widetilde{\mathcal{C}}_{b_{k}}^{-+} u\left(x^{\prime}\right)= & \frac{1}{4} \int_{\Gamma}\left\{\gamma_{a_{k}}^{-} u\left(x^{\prime}+y^{\prime}\right)-\gamma_{b_{k}}^{+} u\left(x^{\prime}\right)\right\} V_{k}\left(x^{\prime}, x^{\prime}+y^{\prime}\right) \mathrm{d} y^{\prime},
\end{aligned}
$$

where $\xi=a_{k}$ or $b_{k}$.

Theorem 6.4. - Assume (6.2), (6.3), and (6.4). Let $f \in \widehat{C}(\bar{E})$ and $\lambda>0$. Then $u=G_{\lambda} f$ is the unique element in $\widehat{C}(\bar{E}) \cap \mathcal{F}$ satisfying the following equations in weak sense: for every $k=0, \pm 1, \pm 2, \ldots$,

$$
\begin{aligned}
\left(\lambda m_{k}-\mathcal{A}\right) u & =m_{k} f & & \text { in } E_{k}, \\
\partial_{n_{b_{k}}^{+}} u & =\widetilde{\mathcal{A}}_{b_{k}}^{+} u+\widetilde{\mathcal{B}}_{b_{k}}^{+} u+\widetilde{\mathcal{C}}_{b_{k}}^{-+} u & & \text { in } \Gamma_{b_{k}}, \\
\partial_{n_{a_{k+1}}^{-}} u & =\widetilde{\mathcal{A}}_{a_{k+1}}^{-} u+\widetilde{\mathcal{B}}_{a_{k+1}}^{-} u+\widetilde{\mathcal{C}}_{a_{k+1}}^{+-} u & & \text { in } \Gamma_{a_{k+1}} .
\end{aligned}
$$

Remark 6.5. - We say that an element $u \in \mathcal{F}$ satisfies Eqs. (6.13)-(6.15) in the weak sense if the equations

$$
\int_{E_{k}}\left(\lambda m_{k}-\mathcal{A}\right) u(x) v(x) \mathrm{d} x=\int_{E_{k}} m_{k} f(x) v(x) \mathrm{d} x \quad \text { for } v \in H_{0}^{1}\left(E_{k}\right),
$$




$$
\int_{\Gamma} \partial_{n_{b_{k}}^{+}} u\left(x^{\prime}\right) \phi\left(x^{\prime}\right) \mathrm{d} x^{\prime}=\int_{\Gamma}\left(\widetilde{\mathcal{A}}_{b_{k}}^{+} u+\widetilde{\mathcal{B}}_{b_{k}}^{+} u+\widetilde{\mathcal{C}}_{b_{k}}^{-+} u\right)\left(x^{\prime}\right) \phi\left(x^{\prime}\right) \mathrm{d} x^{\prime}
$$

for $\phi \in H^{1}(\Gamma)$ or $H^{1 / 2}(\Gamma)$ according as $\kappa_{b_{k}}>0$ or $\kappa_{b_{k}}=0$

hold for (6.13) and (6.14) and a similar equation for (6.15). More precisely, the integrals in the above are understood as follows. Firstly,

$$
\begin{aligned}
& \int_{E_{k}} \mathcal{A} u(x) v(x) \mathrm{d} x \\
& \quad=-\frac{1}{2} \int_{E_{k}}\left\{\sum_{i, j=1}^{d-1} a^{i j}\left(x^{\prime}\right) \partial_{x^{i}} u(x) \partial_{x^{j}} v(x)+a^{d d}\left(x^{d}\right) \partial_{x^{d}} u(x) \partial_{x^{d}} v(x)\right\} \mathrm{d} x
\end{aligned}
$$

for $v \in H_{0}^{1}\left(E_{k}\right)$. Secondly, let $v=H_{\Gamma \times\left\{b_{k}\right\}} \phi$ and take a sequence $\left\{\rho_{n}\right\} \subset C_{0}^{\infty}(\mathbb{R})$ such that $\rho_{n}(\xi)=1$ for $\left|\xi-b_{k}\right|<1 / n,=0$ for $\left|\xi-b_{k}\right|>2 / n$, and $0 \leqslant \rho_{n} \leqslant 1$. Then

$$
\int_{\Gamma} \partial_{n_{b_{k}}^{+}} u\left(x^{\prime}\right) \phi\left(x^{\prime}\right) \mathrm{d} x^{\prime}=\lim _{n \rightarrow \infty} \frac{1}{2} \int_{E_{k}} a^{d d}\left(x^{d}\right) \partial_{x^{d}} u(x) \partial_{x^{d}}\left(v(x) \rho_{n}\left(x^{d}\right)\right) \mathrm{d} x,
$$

for each $\phi \in H^{1}(\Gamma)$ or $H^{1 / 2}(\Gamma)$. Note that the convergence is secured in the following proof of Theorem 6.4. Further, $v=H_{\Gamma \times\left\{b_{k}\right\}} \phi(x)$ can be replaced by any other $v \in$ $H^{3 / 2}\left(E_{k}\right)$ or $H^{1}\left(E_{k}\right)$ with $\gamma_{b_{k}}^{+} v=\phi$. Thirdly,

$$
\int_{\Gamma} \widetilde{\mathcal{A}}_{b_{k}}^{+} u\left(x^{\prime}\right) \phi\left(x^{\prime}\right) \mathrm{d} x^{\prime}=-\frac{\kappa_{b_{k}}}{2} \int_{\Gamma} \sum_{i, j=1}^{d-1} a^{i j}\left(x^{\prime}\right) \partial_{x^{i}} \gamma_{b_{k}}^{+} u\left(x^{\prime}\right) \partial_{x^{j}} \phi\left(x^{\prime}\right) \mathrm{d} x^{\prime},
$$

for $\phi \in H^{1}(\Gamma)$ if $\kappa_{b_{k}}>0$, and $\int_{\Gamma} \widetilde{\mathcal{A}}_{b_{k}}^{+} u\left(x^{\prime}\right) \phi\left(x^{\prime}\right) \mathrm{d} x^{\prime}=0$ for all $\phi \in H^{1 / 2}(\Gamma)$ otherwise. Finally,

$$
\begin{aligned}
& \int_{\Gamma} \widetilde{\mathcal{B}}_{b_{k}} u\left(x^{\prime}\right) \phi\left(x^{\prime}\right) \mathrm{d} x^{\prime} \\
& =-\frac{1}{8} \iint_{\Gamma \times \Gamma}\left\{\gamma_{b_{k}} u\left(x^{\prime}\right)-\gamma_{b_{k}} u\left(y^{\prime}\right)\right\}\left\{\phi\left(x^{\prime}\right)-\phi\left(y^{\prime}\right)\right\} U_{k}\left(x^{\prime}, y^{\prime}\right) \mathrm{d} x^{\prime} \mathrm{d} y^{\prime}, \\
& \int_{\Gamma} \widetilde{\mathcal{C}}_{b_{k}}^{-+} u\left(x^{\prime}\right) \phi\left(x^{\prime}\right) \mathrm{d} x^{\prime}=-\frac{1}{4} \int_{\Gamma} \int_{\Gamma}\left\{\gamma_{a_{k}}^{-} u\left(y^{\prime}\right)-\gamma_{b_{k}}^{+} u\left(x^{\prime}\right)\right\} V_{k}\left(x^{\prime}, y^{\prime}\right) \mathrm{d} y^{\prime} \phi\left(x^{\prime}\right) \mathrm{d} x^{\prime},
\end{aligned}
$$

for all $\phi \in H^{1}(\Gamma)$ or $H^{1 / 2}(\Gamma)$.

Proof of Theorem 6.4. - We will only prove (6.16) and (6.17). Taking $v \in H_{0}^{1}\left(E_{k}\right)$ in (6.1), we obtain (6.16). Take next $v(x) \rho_{n}\left(x^{d}\right)$ for $v$ in (6.1), where $v=H_{\Gamma \times\left\{b_{k}\right\}} \phi$ and $\rho_{n}$ given in Remark 6.5. We then have, for sufficiently large $n$,

$$
\lambda m_{k} \int_{E_{k}} u(x) v(x) \rho_{n}\left(x^{d}\right) \mathrm{d} x+\frac{1}{2} \int_{E_{k}} \sum_{i, j=1}^{d-1} a^{i j}\left(x^{\prime}\right) \partial_{x^{i}} u(x) \partial_{x^{j}} v(x) \rho_{n}\left(x^{d}\right) \mathrm{d} x
$$




$$
\begin{aligned}
& +\frac{1}{2} \int_{E_{k}} a^{d d}\left(x^{d}\right) \partial_{x^{d}} u(x) \partial_{x^{d}}\left(v(x) \rho_{n}\left(x^{d}\right)\right) \mathrm{d} x \\
& +\frac{\kappa_{b_{k}}}{2} \int_{\Gamma} \sum_{i, j=1}^{d-1} a^{i j}\left(x^{\prime}\right) \partial_{x^{i}} \gamma_{b_{k}}^{+} u\left(x^{\prime}\right) \partial_{x^{j}} \phi\left(x^{\prime}\right) \mathrm{d} x^{\prime} \\
& +\frac{1}{8} \int_{\Gamma \times \Gamma}\left\{\gamma_{\xi} u\left(x^{\prime}\right)-\gamma_{\xi} u\left(y^{\prime}\right)\right\}\left\{\phi\left(x^{\prime}\right)-\phi\left(y^{\prime}\right)\right\} U_{k}\left(x^{\prime}, y^{\prime}\right) \mathrm{d} x^{\prime} \mathrm{d} y^{\prime} \\
& +\frac{1}{4} \int_{\Gamma} \int_{\Gamma}\left\{\gamma_{a_{k}}^{-} u\left(y^{\prime}\right)-\gamma_{b_{k}}^{+} u\left(x^{\prime}\right)\right\} V_{k}\left(x^{\prime}, y^{\prime}\right) \mathrm{d} y^{\prime} \phi\left(x^{\prime}\right) \mathrm{d} x^{\prime} \\
& =\int_{E_{k}} f(x) v(x) \rho_{n}\left(x^{d}\right) \mathrm{d} x .
\end{aligned}
$$

The first two terms and the last term go to 0 as $n \rightarrow \infty$. Hence the limit in (6.18) exists and (6.17) follows in the sense of Remark 6.5.

Finally we give intuitive validity of $\widetilde{\mathcal{B}}_{b_{k}}^{ \pm}$. The expression for $\widetilde{\mathcal{B}}_{a_{k}}^{ \pm}$is similar.

Proposition 6.6. - For each $\left.u \in C_{0}^{\infty}\left(\mathbb{R}^{d}\right)\right|_{E_{k}}$ and $\phi \in C_{0}^{\infty}(\Gamma)$, (6.19) holds in the strict sense.

Proof. - Let $\phi, \varphi \in C_{0}^{\infty}(\Gamma)$. Then

$$
\begin{aligned}
& \iint_{\Gamma \times \Gamma}\left\{\phi\left(x^{\prime}\right)-\phi\left(y^{\prime}\right)\right\}\left\{\varphi\left(x^{\prime}\right)-\varphi\left(y^{\prime}\right)\right\} U_{k}\left(x^{\prime}, y^{\prime}\right) \mathrm{d} x^{\prime} \mathrm{d} y^{\prime} \\
& \quad=\lim _{\varepsilon \downarrow 0} \iint_{\Gamma \times \Gamma \backslash \Delta_{\varepsilon}}\left\{\phi\left(x^{\prime}\right)-\phi\left(y^{\prime}\right)\right\}\left\{\varphi\left(x^{\prime}\right)-\varphi\left(y^{\prime}\right)\right\} U_{k}\left(x^{\prime}, y^{\prime}\right) \mathrm{d} x^{\prime} \mathrm{d} y^{\prime} \\
& \quad \equiv \lim _{\varepsilon \downarrow 0} \Phi_{\varepsilon}
\end{aligned}
$$

where $\Delta_{\varepsilon}=\left\{\left(x^{\prime}, y^{\prime}\right) \in \Gamma \times \Gamma:\left|x^{\prime}-y^{\prime}\right|<\varepsilon\right\}$. Noting that $U_{k}\left(x^{\prime}, y^{\prime}\right)=U_{k}\left(y^{\prime}, x^{\prime}\right)$, we have

$$
\begin{aligned}
\Phi_{\varepsilon}= & -\iint_{\Gamma \times \Gamma \backslash \Delta_{\varepsilon}} \phi\left(x^{\prime}\right)\left\{\varphi\left(y^{\prime}\right)-\varphi\left(x^{\prime}\right)-\nabla_{x^{\prime}} \varphi\left(x^{\prime}\right) \cdot\left(y^{\prime}-x^{\prime}\right) \chi\left(y^{\prime}-x^{\prime}\right)\right\} \\
& \times U_{k}\left(x^{\prime}, y^{\prime}\right) \mathrm{d} x^{\prime} \mathrm{d} y^{\prime} \\
& -\iint_{\Gamma \times \Gamma \backslash \Delta_{\varepsilon}} \phi\left(y^{\prime}\right)\left\{\varphi\left(x^{\prime}\right)-\varphi\left(y^{\prime}\right)-\nabla_{y^{\prime}} \varphi\left(y^{\prime}\right) \cdot\left(x^{\prime}-y^{\prime}\right) \chi\left(x^{\prime}-y^{\prime}\right)\right\} \\
& \times U_{k}\left(x^{\prime}, y^{\prime}\right) \mathrm{d} x^{\prime} \mathrm{d} y^{\prime} \\
& -\iint_{\Gamma \times \Gamma \backslash \Delta_{\varepsilon}} \phi\left(x^{\prime}\right) \nabla_{x^{\prime}} \varphi\left(x^{\prime}\right) \cdot\left(y^{\prime}-x^{\prime}\right) \chi\left(y^{\prime}-x^{\prime}\right) U_{k}\left(x^{\prime}, y^{\prime}\right) \mathrm{d} x^{\prime} \mathrm{d} y^{\prime} \\
& -\underset{\Gamma \times \Gamma \backslash \Delta_{\varepsilon}}{ } \phi\left(y^{\prime}\right) \nabla_{y^{\prime}} \varphi\left(y^{\prime}\right) \cdot\left(x^{\prime}-y^{\prime}\right) \chi\left(x^{\prime}-y^{\prime}\right) U_{k}\left(x^{\prime}, y^{\prime}\right) \mathrm{d} x^{\prime} \mathrm{d} y^{\prime}
\end{aligned}
$$




$$
\begin{aligned}
= & -2 \iint_{\Gamma \times \Gamma \backslash \Delta_{\varepsilon}} \phi\left(x^{\prime}\right)\left\{\varphi\left(y^{\prime}\right)-\varphi\left(x^{\prime}\right)-\nabla_{x^{\prime}} \varphi\left(x^{\prime}\right) \cdot\left(y^{\prime}-x^{\prime}\right) \chi\left(y^{\prime}-x^{\prime}\right)\right\} \\
& \times U_{k}\left(x^{\prime}, y^{\prime}\right) \mathrm{d} x^{\prime} \mathrm{d} y^{\prime} \\
& -2 \iint_{\Gamma \times \Gamma \backslash \Delta_{\varepsilon}} \phi\left(x^{\prime}\right) \nabla_{x^{\prime}} \varphi\left(x^{\prime}\right) \cdot\left(y^{\prime}-x^{\prime}\right) \chi\left(y^{\prime}-x^{\prime}\right) U_{k}\left(x^{\prime}, y^{\prime}\right) \mathrm{d} x^{\prime} \mathrm{d} y^{\prime} \\
\equiv & -2 \Phi_{1, \varepsilon}-2 \Phi_{2, \varepsilon} .
\end{aligned}
$$

By means of Lemma 5.2 and Proposition 6.2, there exist the limits of $\Phi_{i, \varepsilon}, i=1,2$, as $\varepsilon \downarrow 0$ and

$$
\begin{aligned}
\lim _{\varepsilon \downarrow 0} \Phi_{1, \varepsilon}= & \int_{\Gamma} \phi\left(x^{\prime}\right) \mathrm{d} x^{\prime} \int_{\Gamma}\left\{\varphi\left(x^{\prime}+y^{\prime}\right)-\varphi\left(x^{\prime}\right)-\nabla_{x^{\prime}} \varphi\left(x^{\prime}\right) \cdot y^{\prime} \chi\left(y^{\prime}\right)\right\} \\
& \times U_{k}\left(x^{\prime}, x^{\prime}+y^{\prime}\right) \mathrm{d} y^{\prime}, \\
\Phi_{2, \varepsilon}= & \int_{\Gamma} \phi\left(x^{\prime}\right) \mathrm{d} x^{\prime} \int_{\Gamma \backslash \Delta_{\varepsilon}^{x^{\prime}}} \nabla_{x^{\prime}} \varphi\left(x^{\prime}\right) \cdot\left(y^{\prime}-x^{\prime}\right) \chi\left(y^{\prime}-x^{\prime}\right) U_{k}\left(x^{\prime}, y^{\prime}\right) \mathrm{d} y^{\prime} \\
= & \int_{\Gamma} \phi\left(x^{\prime}\right) \sum_{i=1}^{d-1} \partial_{x^{i}} \varphi\left(x^{\prime}\right) \mathrm{d} x^{\prime} \int_{\Gamma \backslash \Delta_{\varepsilon}^{x^{\prime}}}\left(y^{i}-x^{i}\right) \chi\left(y^{\prime}-x^{\prime}\right) U_{k}\left(x^{\prime}, y^{\prime}\right) \mathrm{d} y^{\prime} \\
& \longrightarrow \int_{\Gamma} \phi\left(x^{\prime}\right) \sum_{i=1}^{d-1} \partial_{x^{i}} \varphi\left(x^{\prime}\right) b_{k}^{i}\left(x^{\prime}\right) \mathrm{d} x^{\prime} \quad \text { as } \varepsilon \downarrow 0 .
\end{aligned}
$$

Therefore we obtain (6.19).

\section{Acknowledgement}

The authors would like to thank the anonymous referee for the valuable comments.

\section{REFERENCES}

[1] Adams R.A., Sobolev Spaces, Academic Press, New York, 1975.

[2] Albeverio S., Kusuoka S., Streit L., Convergence of Dirichlet forms and associated Schrödinger operators, J. Funct. Anal. 68 (1986) 130-148.

[3] Aronson D.G., Bounds for the fundamental solution of a parabolic equation, Bull. Amer. Math. Soc. 73 (1967) 890-896.

[4] Aronson D.G., Serrin J., Local behavior of solutions of quasilinear parabolic equations, Arch. Rational Mech. Anal. 25 (1967) 81-122.

[5] Feller W., The parabolic differential equations and the associated semi-groups of transformations, Ann. of Math. 55 (1952) 468-519.

[6] Fukushima M., Oshima Y., On the skew product of symmetric diffusion processes, Forum Math. 1 (1989) 103-142.

[7] Fukushima M., Oshima Y., Takeda M., Dirichlet Forms and Symmetric Markov Processes, Walter de Gruyter, Berlin, 1994. 
[8] Hashimoto Y., Manabe S., Ogura Y., Short time asymptotics and an approximation for heat kernel of a singular diffusion, in: Ikeda N., Watanabe S., Fukushima M., Kunita H. (Eds.), Itô's Stochastic Calculus and Probability Theory, Springer, 1996, pp. 129-139.

[9] Itô K., McKean H.P. Jr., Diffusion Processes and their Sample Paths, 2nd edn., Springer, 1974.

[10] Kasue A., Kumura H., Ogura Y., Convergence of heat kernels on a compact manifold, Kyushu J. Math. 51 (1997) 453-524.

[11] Kunita H., General boundary conditions for multi-dimensional diffusion processes, J. Math. Kyoto Univ. 10 (1970) 273-335.

[12] Kuwae K., Uemura T., Weak convergence of symmetric diffusion processes, Probab. Theory Related Fields 109 (1997) 159-187.

[13] Ladyženskaya O.A., Solonnikov V.A., Ural'ceva N.N., Linear and Quasilinear Equations of Parabolic Type, Transl. Math. Monogr., American Mathematical Society, 1968.

[14] Lyons T.J., Zhang T.S., Note on convergence of Dirichlet processes, Bull. London Math. Soc. 25 (1993) 353-356.

[15] Mosco U., Composite media and asymptotic Dirichlet forms, J. Funct. Anal. 123 (1994) 368-421.

[16] Nash J., Continuity of solutions of parabolic and elliptic equations, Amer. J. Math. 80 (1958) 931-954.

[17] Ogura Y., Taniguchi S., A probabilistic scheme for collapse of metrics, J. Math. Kyoto Univ. 36 (1996) 73-92.

[18] Ogura Y., Tomisaki M., Tsuchiya M., Superposition of diffusion processes - Feller property, in: Trends in Probability and Related Analysis, World Scientific, 1999, pp. 113-128.

[19] Ogura Y., Tomisaki M., Tsuchiya M., Existence of a strong solution for an integrodifferential equation and superposition of diffusion processes, in: Stochastics in Finite/Infinite Dimensions (In honor of Gopinath Kallianpur), Birkhäuser, 2001, pp. 341359.

[20] Portenko N.I., Diffusion in a medium with a semi-transparent diaphragms, in: Stochastic Processes and Related Topics (Stochastic Monographs Vol. 10), Gordon and Breach, 1996, pp. 117-128.

[21] Stampacchia G., Equations elliptiques du second ordre à coefficients discontinus, Séminaire sur les equations aux dérivées partielles, Collège de France, 1963. 